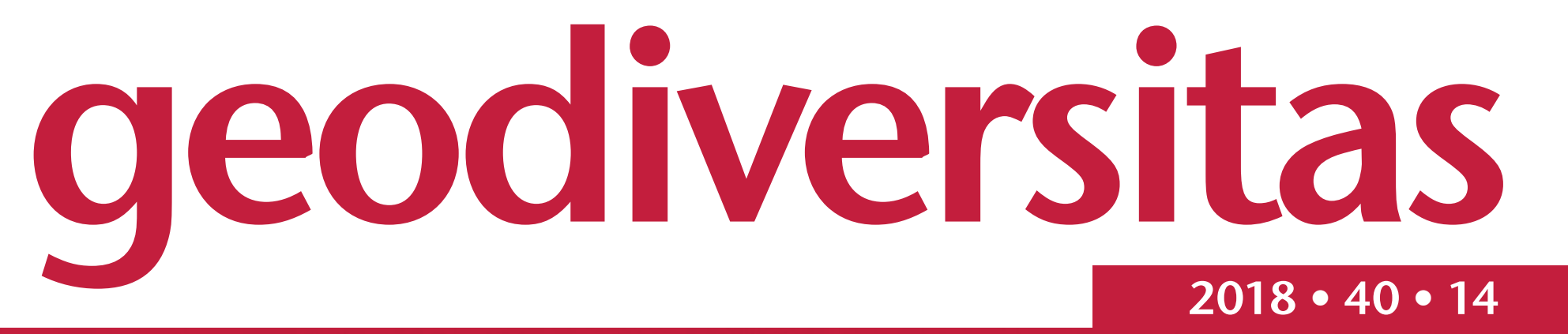

\title{
Biodiversité des coraux scléractiniaires du Langhien (Badénien, Miocène moyen) de Lăpugiu de Sus (Roumanie)
}

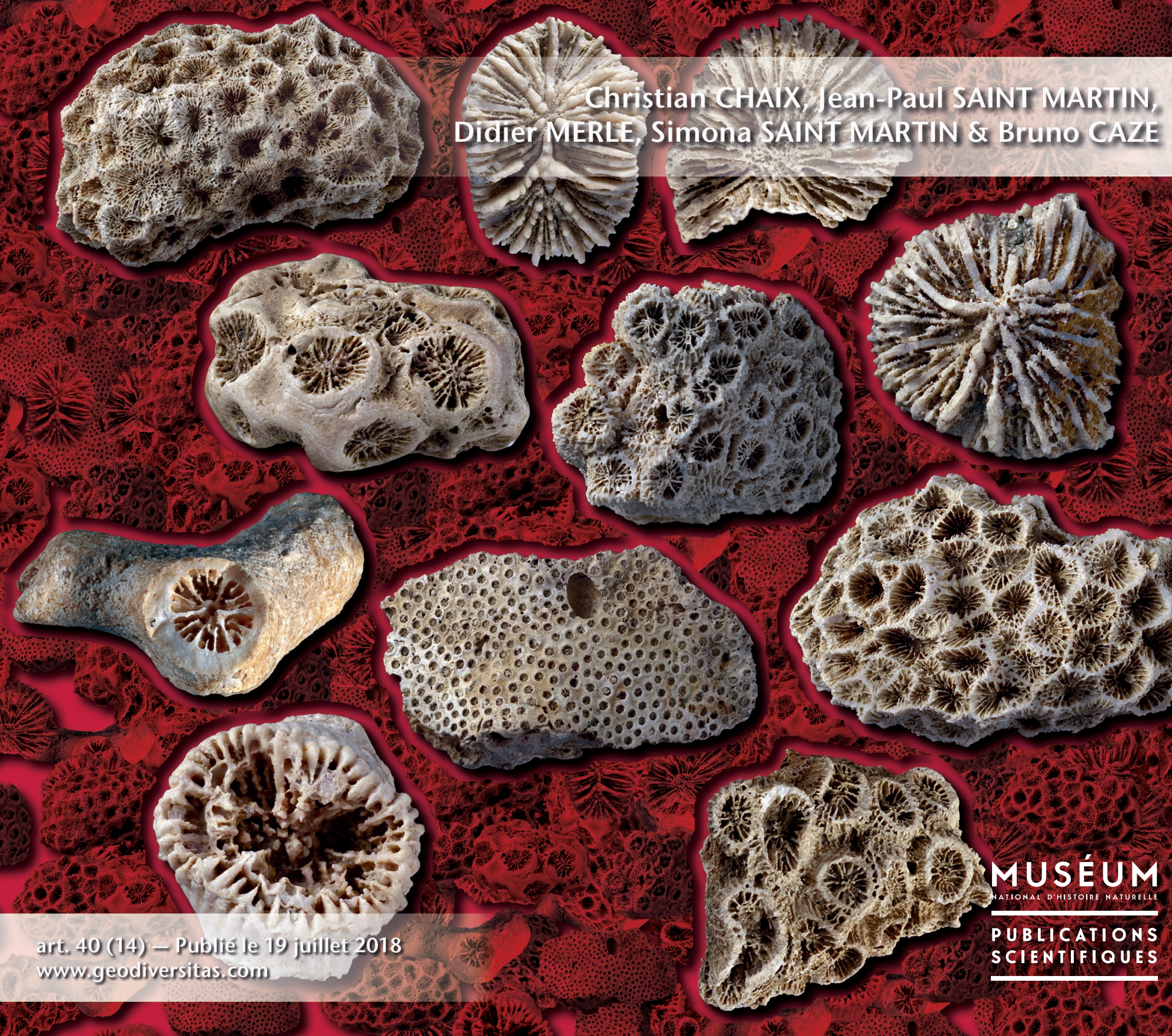


DiRECTEUR DE LA PUBLICATION: Bruno David,

Président du Muséum national d'Histoire naturelle

RÉdACTEUR EN CHEF / EDITOR-IN-CHIEF: Didier Merle

ASSISTANTS DE RÉDACTION / AsSISTANT EDITORS: Emmanuel Côtez (geodiv@mnhn.fr); Anne Mabille

Mise EN PAGE / PAGE LAYOUT: Emmanuel Côtez

COMITÉ SCIENTIFIQUE / SCIENTIFIC BOARD:

Christine Argot (MNHN, Paris)

Beatrix Azanza (Museo Nacional de Ciencias Naturales, Madrid)

Raymond L. Bernor (Howard University, Washington DC)

Alain Blieck (USTL, Villeneuve d'Ascq)

Henning Blom (Uppsala University)

Jean Broutin (UPMC, Paris)

Gaël Clément (MNHN, Paris)

Ted Daeschler (Academy of Natural Sciences, Philadelphie)

Bruno David (MNHN, Paris)

Gregory D. Edgecombe (The Natural History Museum, Londres)

Ursula Göhlich (Natural History Museum Vienna)

Jin Meng (American Museum of Natural History, New York)

Brigitte Meyer-Berthaud (CIRAD, Montpellier)

Zhu Min (Chinese Academy of Sciences, Pékin)

Isabelle Rouget (UPMC, Paris)

Sevket Sen (MNHN, Paris)

Stanislav Štamberg (Museum of Eastern Bohemia, Hradec Králové)

Paul Taylor (The Natural History Museum, Londres)

COUVERTURE / COVER:

Réalisée à partir des Figures de l'article/created from the Figures of this article.

Geodiversitas est indexé dans / Geodiversitas is indexed in:

- Science Citation Index Expanded (SciSearch ${ }^{\circledR}$ )

- ISI Alerting Services ${ }^{\circledR}$

- Current Contents ${ }^{\circledR}$ / Physical, Chemical, and Earth Sciences ${ }^{\circledR}$

- Scopus ${ }^{\circledR}$

Geodiversitas est distribué en version électronique par / Geodiversitas is distributed electronically by:

- BioOne ${ }^{\circledR}$ (http://www.bioone.org)

Les articles ainsi que les nouveautés nomenclaturales publiés dans Geodiversitas sont référencés par / Articles and nomenclatural novelties published in Geodiversitas are referenced by:

- ZooBank ${ }^{\circledR}$ (http://zoobank.org)

Geodiversitas est une revue en flux continu publiée par les Publications scientifiques du Muséum, Paris Geodiversitas is a fast track journal published by the Museum Science Press, Paris

Les Publications scientifiques du Muséum publient aussi / The Museum Science Press also publish: Adansonia, Zoosystema, Anthropozoologica, European Journal of Taxonomy, Naturae.

Diffusion - Publications scientifiques Muséum national d'Histoire naturelle

CP $41-57$ rue Cuvier F-75231 Paris cedex 05 (France)

Tél. : 33 (0)1 40794805 / Fax: 33 (0)1 40793840

diff.pub@mnhn.fr / http://sciencepress.mnhn.fr

(C) Publications scientifiques du Muséum national d'Histoire naturelle, Paris, 2018

ISSN (imprimé / print): 1280-9659/ ISSN (électronique / electronic): 1638-9395 


\title{
Biodiversité des coraux scléractiniaires du Langhien (Badénien, Miocène moyen) de Lăpugiu de Sus (Roumanie)
}

\author{
Christian CHAIX \\ Jean-Paul SAINT MARTIN \\ Didier MERLE \\ Simona SAINT MARTIN \\ Bruno CAZE \\ CR2P (CNRS, MNHN, UPMC, Sorbonne Université), \\ Centre de Recherches sur la Paléobiodiversité et les Paléoenvironnements, \\ Muséum national d'Histoire naturelle, Département Origines et Évolution, \\ case postale 38, 57 rue Cuvier, F-75231 Paris cedex 05 (France) \\ scleractiniaire@live.fr \\ jpsmart@mnhn.fr (auteur correspondant) \\ didier.merle@mnhn.fr \\ simsmart@mnhn.fr \\ bruno.caze@mnhn.fr
}

Soumis le 9 janvier 2018 | accepté le 27 mars 2018 | publié le 19 juillet 2018

MOTS CLÉS Coraux,

Miocène moyen, Langhien,

Paratéthys,

Roumanie,

signalements nouveaux.

Chaix C., Saint Martin J.-P., Merle D., Saint Martin S. \& Caze B. 2018. - Biodiversité des coraux scléractiniaires du Langhien (Badénien, Miocène moyen) de Lăpugiu de Sus (Roumanie). Geodiversitas 40 (14): 321-353. https://doi. org/10.5252/geodiversitas2018v40a14. http://geodiversitas.com/40/14

\section{RÉSUMÉ}

Le gisement de Lăpugiu de Sus (Sud-Ouest de la Roumanie) est renommé pour la richesse et la diversité de la faune préservée dans les couches argilo-sableuses du Langhien (Miocène moyen). La faune corallienne y est également bien représentée. Sur la base des travaux réalisés depuis le 19 ème siècle et de récentes récoltes de matériel, une révision en est proposée. Elle permet d'établir une liste d'environ 65 espèces dont 22 sont nouvellement signalées. Le cadre environnemental est discuté. L'exceptionnelle biodiversité corallienne du gisement, unique pour la Paratéthys, est replacée dans son cadre paléoclimatique et paléogéographique.

\section{ABSTRACT}

Biodiversity of scleractinian corals from the Langhian (Badenian, Middle Miocene) deposits of Lăpugiu de Sus (Romania).

The deposit of Lăpugiu de Sus (South-West of Romania) is famous for the richness and diversity of the invertebrate fauna preserved wthin clayey-sandy layers from Langhian (Middle Miocene). Coral

KEY WORDS

Corals,

Middle Miocene,

Langhian,

Paratethys,

Roumanie,

new records. fauna is also very well represented here. On the basis of work done since the 19th century and new material sampling, a revision is proposed. It allows to establish a list of about 65 species of which 22 are newly reported. The environmental framework is discussed. The exceptional coral biodiversity of the deposit, unique for the Paratethys, is integrated in the palaeoclimatic and palaeogeographic framework. 


\section{EXTENDED ABSTRACT}

The Langhian outcrops of the Lăpugiu de Sus village in Romania (province of Deva) are famous for its richness in fossils with very well-preserved marine shells. Among the many groups represented, corals appear to be the most visible in the field, with some colonies reaching a decimetric size. The fossiliferous deposit of Lăpugiu is located in the neogene Făget basin between the Apuseni Mountains in the North and the Poiana Ruscă mountains in the South. The Făget basin thus forms a passage between the Pannonian basin and the Transylvanian basin, all basins belonging to the vast epicontinental ocean that constituted the Paratethys during the Middle Miocene. The most studied and easily accessible site for fossils sampling including corals is Valea Coşului located in the western part of the village. The surrounding reliefs are made of ante-Neogene material and in some places the contact between the ante-Neogene formations and the Neogene infilling can be observed. An indicative lithographic section studied near the small bridge over the stream Coşu shows an alternation of clayey blue marls, sometimes very dark, and numerous detrital layers, from sandy to conglomeratic. These layers are often lense-shaped bodies of variable extension that can gully the underlying sediments. Locally the section contains either thin horizons of calcareous algae concretions or thin carbonate intercalations, the latter corresponding to episodic deposits of carbonate muds. In the same area, $2 \mathrm{~km}$ east of Lăpugiu between Roşcani and Panc, a freshly cleared slope allowed sampling of some specimens of coral colonies also taken into account in this work.

From a stratigraphic point of view, fossiliferous deposits have been classically attributed to the Middle Miocene and more particularly to Lower Badenian, partially corresponding to the Mediterranean Langhian. The study of foraminifers has permitted to specify that the Lăpugiu langhian section covers the Lagenidae biozone. The calcareous nannoplankton contains species characteristic of the NN5 zone represented in Valea Coşului by the two subzones NN5a and NN5b. It should be noted that the Lagenidae zone and the NN5 biozone would correspond to the middle part of the Badenian according to recent recalibrations proposed for the Vienna Basin.

Recent field investigations have allowed the observation of coral colonies within sediments and the enrichment of the coral-species list, previously established from several works. After clarification of the coral identification criteria, a revision of the species cited by previous authors was carried out and permitted to establish an updated list of 65 species, 22 of which were newly reported.

The abundance of hermatypic corals in the succession of the Lăpugiu Badenian naturally reflects the establishment of favorable tropical climatic conditions. This warm palaeoclimatic context has also been put forward from various data. The mollusc fauna, especially gastropods, exhibits a wide diversity of clearly warm-water taxa (Persististrombus Kronenberg \& Lee, 2007, Tibia Gabb, 1868, Xenophora Troschel, 1852, Cassis Scopoli, 1777, Aspa H. Adams \& A. Adams, 1853, Ficus Röding, 1798, Chicoreus (Triplex) Perry, 1810, Pterynotus Swainson, 1833, Homalocantha Mörch, 1852, Vitularia Swainson, 1840, Melongena Schumacher, 1817, Tarantinaea Monterosato, 1917, Lyria Gray, 1847, Athleta Conrad, 1853, Oniscidia Mörch, 1852, Anazola Gray, 1853, Amalda H. Adams \& A. Adams, 1853 and several genera of cancellarids, terebrids) which could indicate of water temperature higher than $21^{\circ} \mathrm{C}$. More generally, the lower Badenian was marked in Central Paratethys by a development of thermophilic species. Indices of warm waters are also given by calcareous nannoplankton and the presence of ascidia spicules specific to reef environments. Palynological data also show predominance of thermophilic elements in the pollen spectrum. All these indications in the trend of a warm episode, even a subtropical episode, are probably related to the climatic optimum of the Middle Miocene evidenced in the whole Paratethys. From the palaeoceanographic point of view, the warm phase of the early Badenian period coincides with the invasion of Indo-Mediterranean fauna. This period would be marked by the reopening of communications with Indo-Pacific ocean realm causing a warming of waters of about $3-4^{\circ}$, naturally favorable to the development of a subtropical fauna such as the hermatypic corals.

The abundance and the diversity of hermatypic corals in the Lăpugiu strata classically indicate very shallow waters. The information provided by several groups of organisms represented in the whole fauna, such as molluscs, corroborate this fact, which is essential for the reconstitution of the palaeoenvironment. The mollusc's assemblage clearly indicates the infralittoral zone and thus the euphotic zone. It should be noted that the growth of a large variety of corals does not mean evolution towards a reefal environment properly speaking. The corals of the Lăpugiu site reflect probably a non-framework coral community characterized only by lateral juxtaposition of colonies and by the absence of vertical stacking. Such a characteristic is also observed within the coral deposits of the Miocene of Aquitaine (France).

The field data and the composition of the fauna preserved in Lăpugiu therefore suggest an unstable coastal environment where varied coral species grew, but represented by rather small colonies. This instability does not allow the aggregation in time of the colonies and therefore the formation of reefs. The coastal palaeogeography could comprises shallow bays notched by streams. The frequency of 
terrigenous episodes indicates that these coastal environments were subjected to recurrent sudden discharges of continental material into the marine environment and to remodeling of the coastal line. The abundance of plants remains, more or less lignified according to the case, also reflects this continental influence.

The high coral biodiversity of Lapugiu is exceptional for a single site, with 42 species of z-corals (corals with zooxanthellae) and 21 species of non-reef solitary and colonial corals. No other Miocene basin in Romania has such diversity. If the coral biodiversity of Lăpugiu appears remarkable in the context of the Neogene basins of Romania, it is interesting to extend the palaeogeographic field to the central Paratethys. Generally related to Leithakalk facies, coral faunas have been recorded in many countries with latitudinal amplitude from Bulgaria to Poland. The compilation of all data reveals a significantly larger coral biodiversity in Lăpugiu, especially considering that it concerns only one relatively limited fossil site in space. However, the biodiversity of hermatypic corals in Leithakalk-type facies in Romania could be underestimated due to the particular taphonomic and diagenetic conditions of the carbonate environments rather unfavorable to good preservation of the diagnostic characteristics of coral skeletons. Taphonomic conditions may have played a role in the fact that the coral colonies, as well as the shells of molluscs, were quickly displaced before degradation, redistributed to more protected and less oxygenated bay bottoms, and finally covered with clay mud allowing a better preservation of aragonite. The latitudinal position could also be a factor of diversification, the coral reefs being further south and assemblages of hermatypical corals appearing to be poorer to the North. From this point of view, the southern situation of the Făget basin could partially explain a certain differentiation, but only in relation to the basins situated much farther north (Hungary, Austria, Poland ...). Thus, Lăpugiu-type deposits may be considered to reflect the best-preserved Badenian coral palaeocommunity. However, it is worth noting that the coral fauna listed more south (Bulgaria for example) do not show more diversity. The special palaeogeography of the Lăpugiu region within palaeoreliefs forming spaced lands could have constituted a context of archipelagos, the mutiplication of islands favoring the development of coastal fauna. On the other hand, the position of the Făget basin in a communication channel must also allow circulation and exchange of larvae conducive to coral implantation on the extensive coastal areas. Finally, the coral diversity of Lăpugiu is also related to a wide variety of biotopes and substrates in environments ranging from infralittoral to circalittoral, which stands are often mixed or intercalated in a series that is finally thin.

The coral fauna of Lăpugiu de Sus should be placed more largely in the context of the mid-Miocene seas characterized by coral abundance and the acme of reef development linked to the global Middle Miocene Climatic Optimum. This period also corresponds to the most northerly extension of coral buildings. As a result, the taxonomic richness of Lăpugiu in z-genera, which until now has not been well taken into account, may be more related with expected climatic optimum. The information obtained from this deposit alone constitutes a necessary reference for any understanding of the palaeotemperature and palaeoceanography of this Middle Miocene period. Finally, coral biodiversity in z-genera and non-z-genera appears to be the most important of what is known in Paratethys. The remarkable coral biodiversity highlighted in Lăpugiu must also be related to the exceptional biodiversity observed for other groups. For example, molluscs have attracted attention with an impressive list of several hundred species described or cited by several authors since the 19th century. Whatever the various factors contributing to this fossil richness, it can legitimately be considered that the site of Lăpugiu corresponds fairly well to the criteria that determine the qualification of hot spot of fossil biodiversity. The coral biodiversity highlighted in this work would thus constitute an element to be taken largely into account in this perspective.

\section{INTRODUCTION}

Le site miocène de Lăpugiu de Sus, situé dans la province de Deva en Roumanie, est renommé depuis longtemps pour sa richesse en fossiles aux coquilles très bien conservées (Hörnes 1854 ; Stur 1863). On doit en fait sa découverte à Edward Albert Bielz en 1845 (in Stur 1863).

Parmi les très nombreux groupes représentés, les coraux apparaissent comme les plus repérables sur le terrain, certaines colonies pouvant atteindre des tailles décimétriques. Le premier travail important portant sur les scléractiniaires est le fait de Reuss (1871) qui propose une description et une figuration d'échantillons de nombreux gisements d'Autriche-Hongrie. Il dresse ainsi une liste de 18 espèces provenant plus spécialement de Lăpugiu de Sus (= Lapugy), ce qui en fait le site le plus riche. Par la suite Neugeboren (1876) apporte des compléments avec des exemplaires de diverses collections, dont celles du 


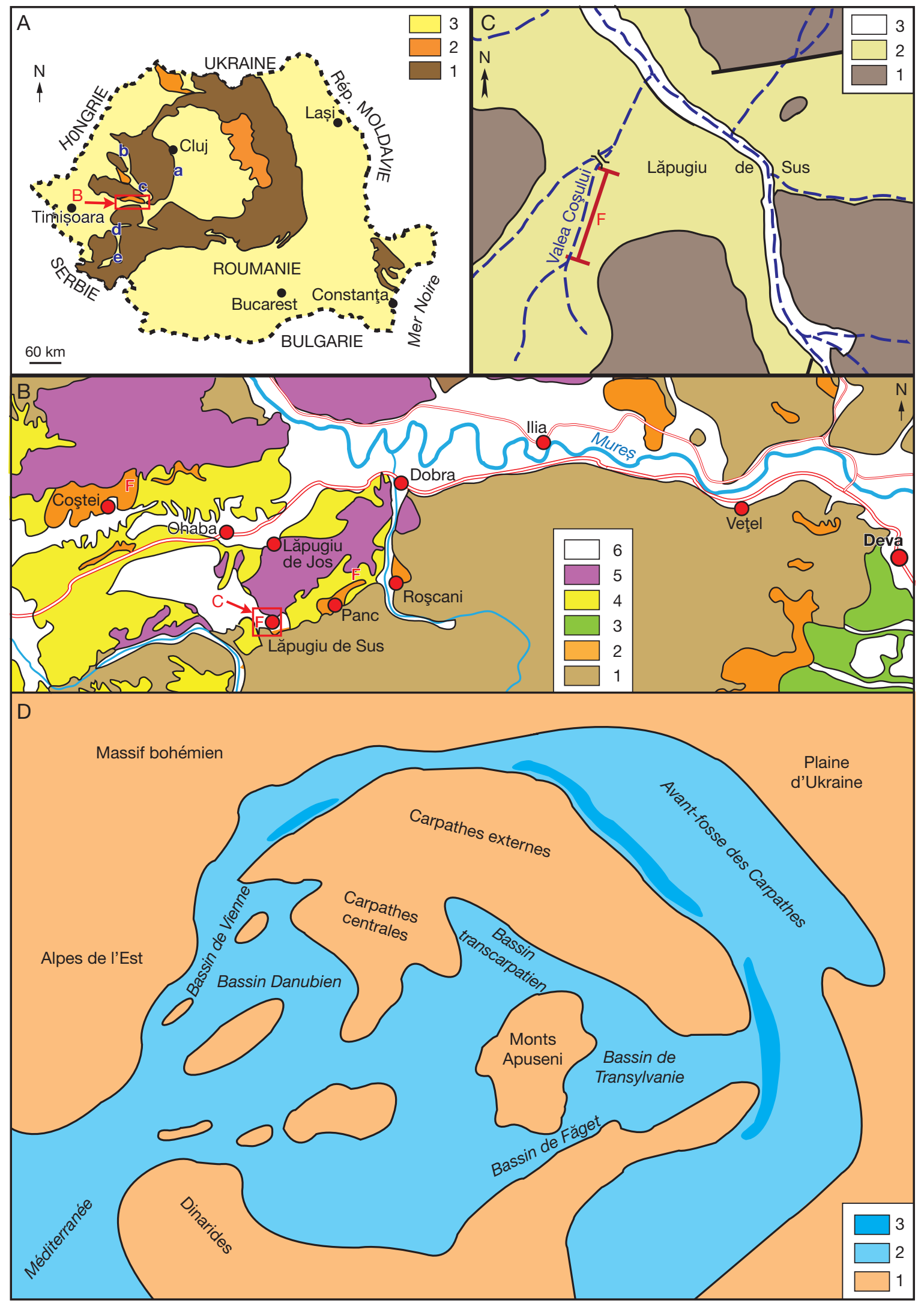

FIG. 1. - A, Carte géologique sommaire de la Roumanie, emplacement de la zone étudiée et situation des principaux bassins renfermant des coraux du Miocène moyen (d'après Saint Martin et al. 2007) : 1, substratum anté-néogène; 2, volcanisme; 3, Néogène-Quaternaire; a, Transylvanie occidentale; b, Bassin Zarand; c, Bassin de Beiuș; d, Bassin de Bahna; e, Bassin de Mehadia; B, carte géologique de la région de Deva (d'après la carte géologique au 1/200 000 de Deva): 1, Terrains anté-néogènes; 2, Badénien; 3, Sarmatien; 4, Pannonien; 5, volcanisme néogène-quaternaire; 6, Quaternaire; C, carte géologique simplifiée du secteur de Lăpugiu de Sus: 1, terrains anté-néogènes et terrains volcaniques; 2, Badénien fossilifère; 3, Quaternaire-Récent; F, zones de prélèvement de colonies coralliennes; D, carte paléogéographique de la Paratéthys centrale au Badénien (d’après Kováč et al. 2017, simplifiée). 
Musée Bruckenthal ou d'une association naturaliste de Sibiu (= Hermannstadt). Cet auteur ne propose pas de figuration des 28 espèces décrites, ne s'appuyant curieusement que sur celles du travail de Reuss (1871). Plusieurs spécimens ne sont pas déterminés spécifiquement. Une liste synthétique des coraux connus de Lăpugiu est établie par Koch (1900) à partir des travaux de Reuss (1871), de Neugeboren (1876) et de quelques compléments de Halaváts (1876) avec alors 37 espèces recensées. Aucune étude n'est ensuite consacrée aux coraux de Lăpugiu jusqu’à Kolosváry (1961) qui décrit seulement 3 espèces parmi des exemplaires déposés dans la collection de l'Institut Géologique de Hongrie. Enfin, plus récemment, Rus \& Popa (2008) ont décrit et figuré 24 espèces de coraux parmi les exemplaires de coraux provenant des collections du Musée de Paléontologie-Stratigraphie de l'Université Babeş-Bolyai de Cluj-Napoca.

De nouvelles investigations sur le terrain ont permis d'observer les colonies in situ dans les sédiments et d'enrichir la liste des espèces coralliennes. Après clarification des critères d'identification des coraux, une révision des espèces citées par les divers auteurs a été effectuée permettant d'établir une liste mise à jour de 65 espèces, dont 22 nouvellement signalées.

\section{CADRE GÉOLOGIQUE}

Le gisement fossilifère du village de Lăpugiu est situé dans le bassin néogène de Făget entre les Monts Apuseni au Nord et les Monts de Poiana Ruscă au Sud (Fig. 1A-C), qui constituait ainsi un passage entre le bassin pannonien et le bassin de Transylvanie appartenant au vaste domaine océanique épicontinental que constituait la Paratéthys au Miocène moyen (Fig. 1D). Les principaux affleurements riches en fossiles correspondent aux berges de plusieurs ruisseaux (Şuraru \& Papp 1993). Le site le plus étudié et le plus facile d'accès est celui de Valea Coşului à l'Ouest du village (Fig. 1C). Les reliefs environnants sont constitués de matériel anté-néogène et à certains endroits on peut observer le contact entre les formations anciennes et le remplissage néogène. Plusieurs travaux ont proposé une succession plus ou moins détaillée de la série miocène à Lăpugiu, mettant en évidence la mixité d'une sédimentation argileuse et détritique (Marinescu 1972; Marinescu \& Popescu 1978; Şuraru \& Papp 1993). Effectivement, une coupe indicative levée à proximité du petit pont qui enjambe le ruisseau montre une succession lithologique très variable marquée par une alternance de marnes argileuses bleues, parfois très sombres, et de nombreuses passées détritiques, de sableuses à conglomératiques (Figs 2;3). Il s'agit d'ailleurs souvent plutôt de lentilles de matériel détritique à l'extension variable pouvant raviner nettement les sédiments sous-jacents (Fig. 3C). Localement la série renferme de fins niveaux à dragées d'algues calcaires ou des intercalations carbonatées peu épaisses correspondant à des dépôts épisodiques de vases calcaires.

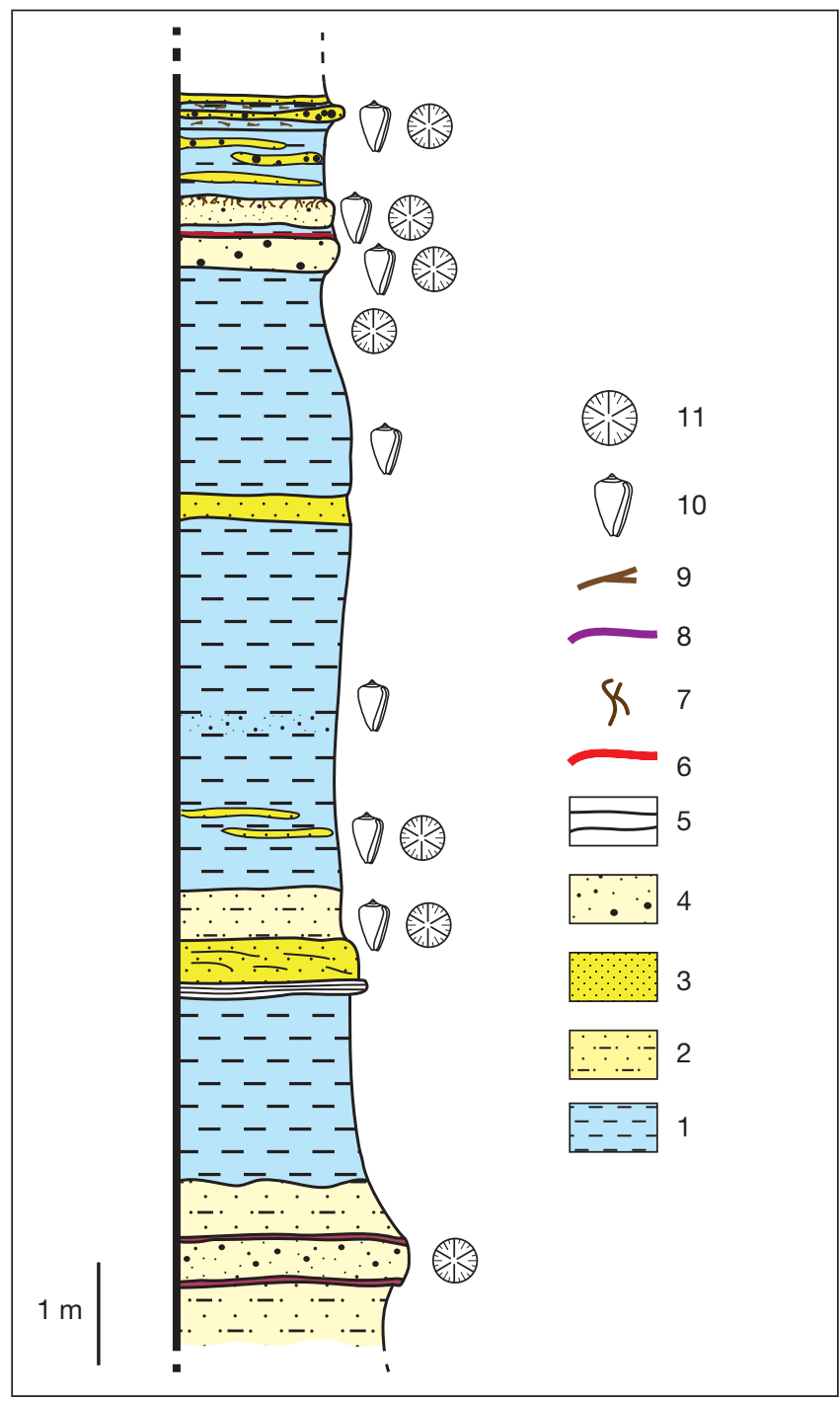

FIG. 2. - Coupe schématique le long de Valea Coșuliu à l'ouest de Lăpugiu de Sus: $\mathbf{1}$, lits argileux à passées sableuses; 2 , marnes sableuses; $\mathbf{3}$, sables; $\mathbf{4}$, lits ou lentilles détritiques, sableuses à conglomératiques; $\mathbf{5}$, bancs carbonatés indurés; 6, surfaces ferrugineuses; $\mathbf{7}$, bioturbations; 8 , lits sombres ligniteux; 9 , débris végétaux plus ou moins lignitisés; $\mathbf{1 0}$, mollusques; 11 , colonies coralliennes.

Dans le même secteur, $2 \mathrm{~km}$ à l'est de Lăpugiu entre Roșcani et Panc (Fig. 1B), un talus fraîchement dégagé a permis la récolte de quelques spécimens de colonies coraliennes également pris en compte dans ce travail (Fig. 4). Au Nord-Ouest le gisement de Costei (Fig. 1B) de Sus renferme surtout des mollusques, mais quelques coraux solitaires peuvent y être récoltés (cf. Reuss 1871).

Du point de vue stratigraphique, les dépôts fossilifères ont été attribués classiquement au Miocène moyen et plus particulièrement au Badénien inférieur (Moravien), correspondant partiellement au Langhien méditerranéen, dans un contexte transgressif (Kovač et al. 2007; Piller et al. 2007; Rögl et al. 2007, 2008; Filipescu 2011; Zuschin et al. 2011; Hohenegger et al. 2014). L'étude des associations de foraminifères a permis de préciser que la série étudiée recouvre ainsi la biozone à Lagenidae (Marinescu \& 

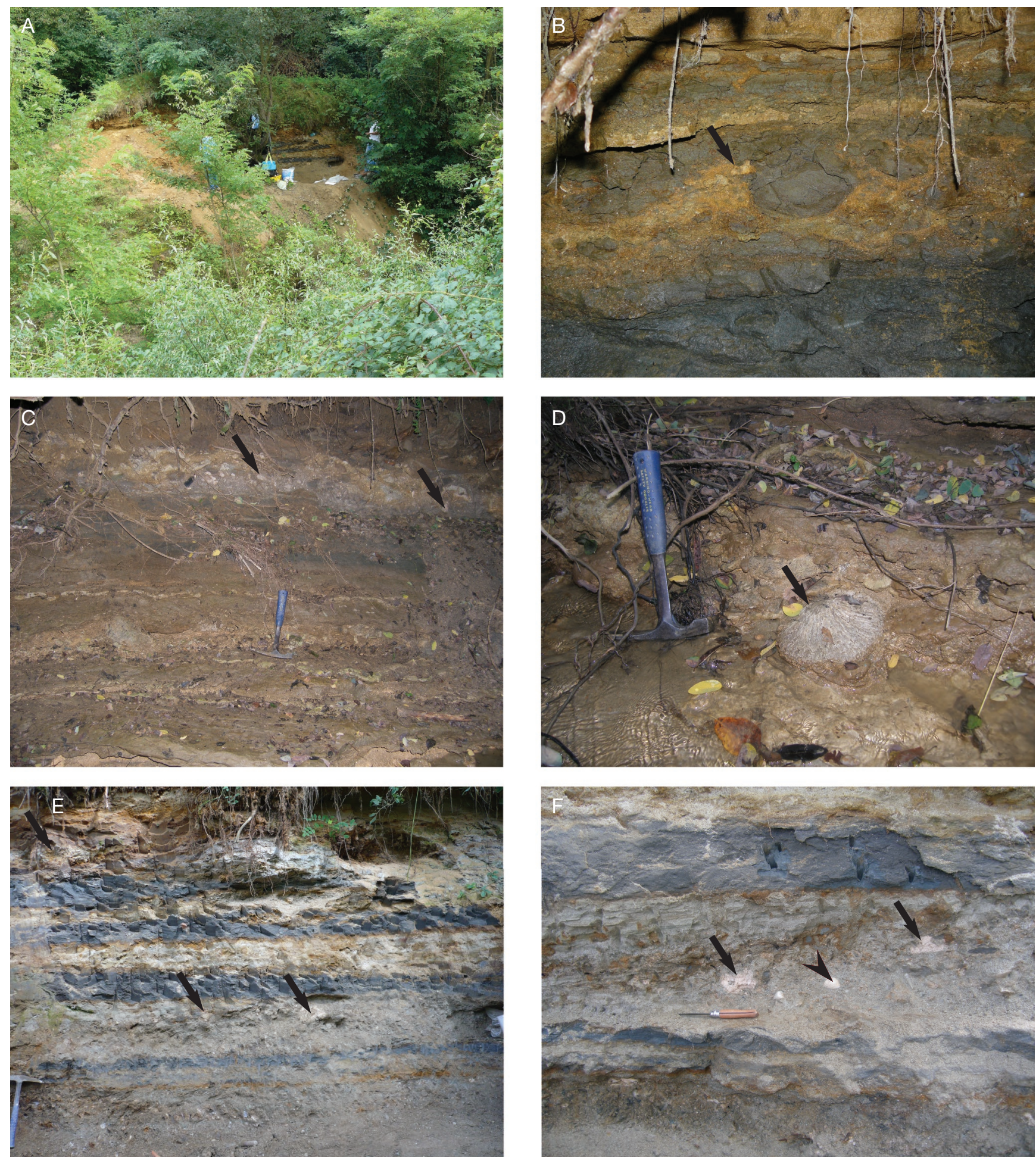

FIG. 3. - A, Vue partielle des affleurements sur la rive droite de Valea Coșuliu; B, lits à galets argileux et débris de coraux (flèche) ; C, alternances argilo-sableuses en bordure du ruisseau renfermant localement des colonies coralliennes (flèches) ; D, colonie corallienne renversée au sein d'un sédiment sableux ; E, alternance d'argiles sombres bleutées et de passées détritiques sableuses à conglomératiques renfermant en abondance des coquilles de mollusques et des colonies ou fragments de colonies coralliennes (flèches) ; $\mathbf{F}$, détail d'un niveau sableux riche en coquilles de mollusques (tête de flèche) et de colonies coralliennes (flèches). Photos J.-P. Saint Martin.

Popescu 1978 ; Şuraru \& Papp 1993; Boga 2013). Le nannoplancton calcaire renferme des espèces caractéristiques de la zone NN5 représentée dans Valea Coşului par les deux sous-zones NN5a et NN5b (Chira \& Marunțeanu 1999,
2000). Il est à noter que la zone à Lagenidae et la biozone NN5 correspondraient à la partie moyenne du Badénien selon les recalibrations proposées par Hohenegger et al. (2014) pour le bassin de Vienne. 


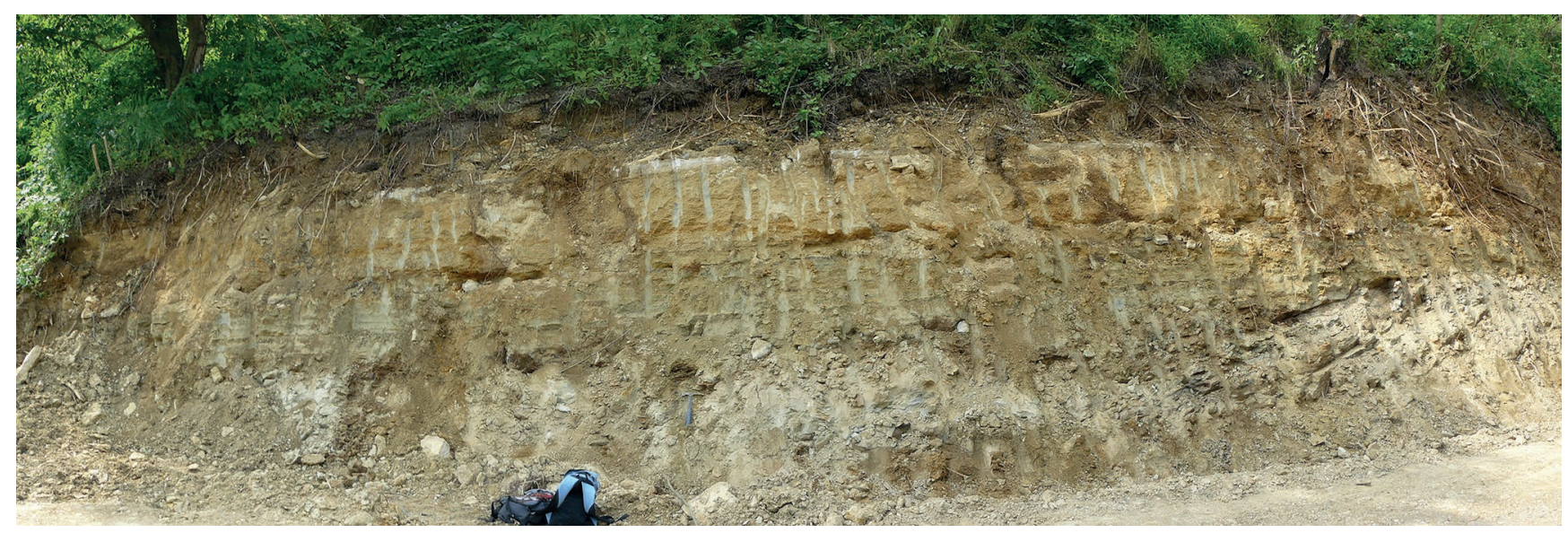

FIG. 4. - Affleurements sur la route de Roșcani à Panc montrant une sédimentation riche en éléments détritiques du substratum anté-néogène et des corps sédimentaires ravinants renfermant des coquilles de mollusques et des colonies coralliennes. Photo J.-P. Saint Martin.

\section{MATÉRIEL ET MÉTHODES}

\section{ORIGINE DU MATÉRIEL}

Le nouveau matériel paléontologique présenté ici a été entièrement récolté in situ. Il s'agit principalement des coraux qui font l'objet de la présente étude systématique et dans une moindre mesure des mollusques déterminés afin de préciser par leur paléoécologie la nature des environnements coralliens. Une partie des échantillons provient du lavage et du tamisage de sédiments en général détritiques et riches en mollusques. Des exemplaires isolés ont été prélevés directement dans le sédiment. Enfin d'autres exemplaires dégagés par l'érosion et le ruissellement ont été ramassés tout au long du ruisseau Coşu.

\section{BASES SYSTÉMATIQUES DES CORAUX}

Les critères d'identification, qu'ils soient génériques ou spécifiques, reposent sur la nature et les variations des différents composants squelettiques: éléments radiaires (septes, côtes, lames biseptales), columelle (nombreuses allures possibles), ornementation septale (dont présence ou absence de pali), nature de la muraille (présente ou absente), existence et allure de l'endothèque et de l'exothèque, caractère compact ou poreux du squelette, et bien entendu le caractère solitaire ou colonial; pour ce dernier, on utilise les rapports géométriques des calices (notamment le rapport $\mathrm{d} / \mathrm{D}$ entre le petit diamètre calicinal et le grand diamètres calicinal), et les relations entre eux (Chaix \& Cahuzac 2005; Chaix \& Saint Martin 2008; Chaix et al. 2015). Cette étude n’intègre pas les techniques d'étude des parties molles (entre autres moléculaires), ces dernières n'étant naturellement pas préservées chez les fossiles. Les caractères génériques sont qualitatifs (présence ou absence d'une structure squelettique, différences d'une même structure) alors que les caractères spécifiques sont quantitatifs (diamètres des calices, espacement entre eux, nombre d'éléments radiaires).

Les identifications de Reuss (1871), Neugeboren (1876) et Rus \& Popa (2008) ont été revues sur ces bases. Plusieurs cas de figure se sont présentés. Dans certains cas l'identification n'était pas exacte et l'espèce a été renommée. Dans d'autres cas, la même espèce a été désignée sous des noms différents selon les exemplaires ou à l'inverse des exemplaires désignés sous des noms d'espèces différentes correspondaient en fait à une seule espèce. Ces données sont résumées dans le Tableau 1.

\section{ÉTUDE SYSTÉMATIQUE}

Famille STYLOPHORIDAe Milne-Edwards \& Haime, 1857

Genre Stylophora Schweigger, 1819

ESPÈCE TYPE. — Madrepora pistillata Esper, 1797.

\section{DESCRIPTION}

Genre colonial, zooxanthellé, vivant en milieu récifal. Colonies souvent branchues, plocoïdes à très petits calices (diamètre calicinal de $3 \mathrm{~mm}$ au maximum). Columelle styliforme liée aux septes, peu nombreux en général.

\section{Stylophora calcinata (Mayer, 1864)}

(Fig. 5A)

Danaia calcinata Mayer, 1864: 191, pl. I, fig. 5.

Matériel. - Trois exemplaires de Roșcani, spécimen figuré: MNHN.F.A59907.

Répartition STRATIGRAPHIQUe ET GÉOGRAPHIQUe. - Miocène moyen: Portugal, Madère (Mayer 1864), Roumanie (Roșcani, ce travail).

\section{DESCRIPTION}

Colonies de forme plus ou moins digitées, à disposition plocoïde des calices (calices circulaires plus ou moins espacés), à squelette entièrement compact (septes, muraille, périthèque, etc.). La columelle est styliforme. Aucune ornementation périthécale n'est visible, contrairement à d'autres espèces. Les 
TABLEAU 1. - Liste des espèces de coraux scléractiniaires du Badénien déterminés à Lăpugiu (Roumanie) à partir de nouvelles récoltes et des travaux antérieurs réalisés à Lăpugiu et sur d'autres sites du Badénien de Paratéthys. Les noms d'espèces dans les listes fournies par les auteurs ont été, autant que possible, mis à jour, parfois révisés, et homogénéisés. Les déterminations douteuses ou établies à partir de sur des caractères peu visibles ou absents ont été retirées des listes. De même les espèces non identifiables d'après les travaux d'origine ont été omises. Symbole: +, présent; -, absent.

\begin{tabular}{|c|c|c|c|c|c|c|c|c|c|c|c|c|c|c|c|}
\hline \multirow[b]{2}{*}{ Coraux scléractiniaires du Badénien de Paratéthys } & \multirow[b]{2}{*}{ 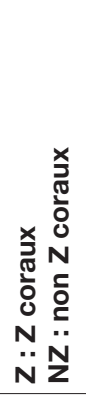 } & \multicolumn{5}{|c|}{ Lăpugiu } & \multicolumn{3}{|c|}{$\begin{array}{l}\text { Autres } \\
\text { gisements } \\
\text { roumains } \\
\end{array}$} & \multicolumn{6}{|c|}{ Ensemble Paratéthys } \\
\hline & & 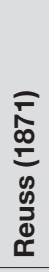 & 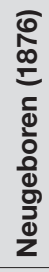 & 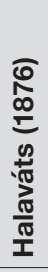 & 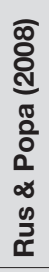 & 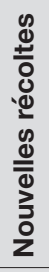 & 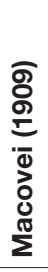 & 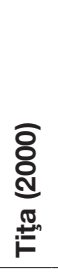 & 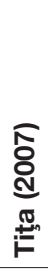 & $\begin{array}{l}\stackrel{0}{\circ} \\
\text { 응 } \\
\frac{0}{0}\end{array}$ & $\frac{0}{\frac{0}{0}}$ & $\begin{array}{l}\frac{0}{J} \\
\frac{0}{\oplus} \\
\frac{0}{0} \\
\end{array}$ & $\begin{array}{l}\text { 은 } \\
\text { 음 } \\
\text { 오 }\end{array}$ & 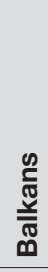 & $\frac{.0}{\frac{0}{\bar{T}}}$ \\
\hline Astrocoenia ornata Duncan, 1863 & Z & - & - & - & - & - & - & - & - & - & - & - & - & - & + \\
\hline Stylophora calcinata (Mayer, 1864) & $\bar{Z}$ & - & - & - & - & + & - & - & - & - & - & - & - & - & - \\
\hline Stylophora depauperata (Reuss, 1867) & Z & - & - & - & + & - & - & - & - & - & - & - & - & - & - \\
\hline Stylophora subreticulata Reuss, 1871 & Z & - & - & - & - & - & - & - & - & - & - & - & + & - & + \\
\hline Stylophora reussiana Montanaro-Gallitelli \&Tacoli, 1951 & Z & - & - & - & - & - & - & - & - & + & - & - & + & - & - \\
\hline Leptoseris? & $\bar{Z}$ & - & - & - & - & - & - & - & - & - & + & - & - & - & - \\
\hline Pavona minor (Zuffardi-Comerci, 1932) & Z & - & - & - & - & - & - & - & - & - & - & - & + & - & - \\
\hline Siderastraea radians (Pallas, 1766) & Z & - & - & - & - & + & - & - & - & - & - & - & - & - & - \\
\hline Siderastraea siderea (Ellis \&Solander, 1786) & Z & - & - & - & - & + & - & - & - & - & - & - & - & - & - \\
\hline Siderastraea italica (Defrance, 1826) & Z & - & - & - & - & - & - & - & - & + & + & - & + & - & - \\
\hline Siderastraea crenulata (Goldfuss, 1826) & Z & - & - & - & - & - & - & - & - & - & + & - & + & - & - \\
\hline Siderastraea felixi Roszkowska, 1932 & Z & - & - & - & + & - & - & - & - & - & - & - & - & - & - \\
\hline Siderastraea lomnickii Roszkowska, 1932 & Z & - & - & - & - & - & - & - & - & - & - & - & - & - & + \\
\hline Stephanophyllia elegans (Bronn, 1838) & $\mathrm{NZ}$ & - & - & - & - & - & + & - & - & + & + & - & - & - & - \\
\hline Stephanophyllia imperialis Michelin, 1841 & NZ & + & + & - & - & - & - & - & - & - & + & - & - & - & - \\
\hline Favia magnifica Reuss, 1871 & Z & + & + & - & - & - & + & - & + & - & + & - & + & - & - \\
\hline Favia somaliensis Gregory, 1900 & Z & - & - & - & + & - & - & - & - & - & - & - & - & - & - \\
\hline Favia gotschevi Kojumdgieva et Strachimirov, 1960 & Z & - & - & - & - & - & - & - & - & - & - & - & - & - & + \\
\hline Favites neglecta (d'Achiardi, 1868) & $\bar{Z}$ & - & - & - & - & + & - & - & - & - & - & - & - & - & - \\
\hline Favites detecta (Michelotti in Sismonda, 1871) & Z & - & - & - & + & + & - & - & - & - & - & - & - & - & - \\
\hline Favites neugeboreni (Reuss, 1871) & Z & + & + & - & + & + & + & - & - & - & - & - & - & + & + \\
\hline Favites conferta (Sismonda, 1871) & Z & - & - & - & + & - & - & - & - & - & - & - & - & - & - \\
\hline Favites cf. oligocenica Chevalier, 1955 & Z & - & - & - & - & + & - & - & - & - & - & - & - & - & - \\
\hline Caulastrea cf. furcata Dana, 1846 & Z & - & - & - & - & - & - & - & - & - & + & - & - & - & - \\
\hline Heliastraea (Aquitanastraea) guettardi (Michelin, 1841) & Z & - & - & - & - & + & - & - & - & - & - & - & - & - & - \\
\hline Heliastraea (Aquitanastraea) ramea (Duncan, 1863) & Z & + & + & - & - & - & + & - & - & - & - & - & + & - & - \\
\hline Heliastraea (Aquitanastraea) incrustans (Osasco, 1897) & Z & - & - & - & - & + & - & - & - & - & - & - & - & - & - \\
\hline Heliastraea (Aquitanastraea) solenastroides (Chevalier, 1954) & Z & - & - & - & - & + & - & - & - & - & - & - & - & - & - \\
\hline Heliastraea (Aquitanastraea) pelouaensis Chevalier, 1954 & Z & - & - & - & - & + & - & - & - & - & - & - & - & - & - \\
\hline Heliastraea (Heliastraea) brevis Duncan, 1863 & Z & - & - & - & - & + & - & - & - & - & - & - & + & - & - \\
\hline Heliastraea (Heliastraea) oligophylla Reuss, 1871 & $\bar{Z}$ & + & + & - & - & - & + & - & - & - & + & - & - & - & - \\
\hline Heliastraea (Heliastraea) saubriguensis Chevalier, 1962 & Z & - & - & - & - & + & - & - & - & - & - & - & - & - & - \\
\hline Heliastraea sp. & Z & - & - & - & - & - & + & - & - & - & - & - & - & - & - \\
\hline Heliastraea (Heliastr & Z & - & - & - & + & - & - & - & - & - & - & - & - & - & - \\
\hline Tarbellastraea ellisiana (Defrance, 1826) & $\bar{Z}$ & + & + & - & - & + & - & - & + & - & + & - & + & - & - \\
\hline Tarbellastraea reussiana (M.E. \& H., 1850) & Z & + & + & + & + & + & + & - & + & + & + & - & + & + & + \\
\hline Tarbellastraea distans Chevalier, 1962 & Z & - & - & - & - & - & - & - & - & - & - & - & - & - & + \\
\hline Antiguastraea aquitaniensis (Chevalier, 1962) & Z & + & + & - & - & + & - & - & - & - & - & - & - & - & - \\
\hline Plesiastraea (Paleoplesiastraea) desmoulinsi (M.E. \& H., 1851) & Z & + & + & - & - & + & + & - & - & + & - & - & + & - & - \\
\hline Plesiastraea (Paleoplesiastraea) tazarinensis (Chevalier, 1962) & Z & - & - & - & - & + & - & - & - & - & - & - & - & - & - \\
\hline Thegioastraea incerta (Osasco, 1897) & Z & - & - & - & - & - & - & - & - & - & - & - & + & - & - \\
\hline Thegioastraea sp. & Z & - & - & - & - & - & - & - & - & - & - & - & + & - & - \\
\hline Solenastrea sp. & Z & - & - & - & - & - & - & - & - & - & - & - & - & - & - \\
\hline Cladocora prevostana M.E. \& H., 1849 & NZ & + & + & - & - & - & - & - & - & - & - & - & - & - & - \\
\hline Cladocora reussi de Fromentel, 1861 & NZ & - & - & - & - & - & - & - & - & - & + & - & - & - & - \\
\hline Stylocora exilis Reuss, 1847 & Z & - & - & - & - & - & - & - & - & + & + & - & - & - & - \\
\hline Cladangia conferta (Reuss, 1847) & Z & - & - & - & + & + & - & - & - & + & + & - & + & - & - \\
\hline Rhizangia procurrens Reuss, 1871 & Z & + & + & - & - & - & - & - & - & - & - & - & + & - & - \\
\hline Oulangia speyeri (Reuss, 1865) & Z & - & - & - & - & + & - & - & - & - & - & - & - & - & - \\
\hline Oculina parvistella Reuss, 1871 & Z & + & + & - & - & - & - & - & - & - & - & - & - & - & - \\
\hline Astrohelia gaillardensis Chevalier, 1962 & Z & - & - & - & - & + & - & - & - & - & - & - & - & - & - \\
\hline Lithophyllia coronula (Michelotti, 1838) & Z & - & - & - & - & + & - & - & - & - & - & - & - & - & - \\
\hline Acanthophyllia ampla (Reuss, 1871) & NZ & + & + & - & - & + & - & - & - & - & + & - & + & - & + \\
\hline Acanthophyllia spinosa (Gerth, 1921) & $\mathrm{NZ}$ & - & - & - & + & - & - & - & - & - & - & - & - & - & - \\
\hline Acanthastraea horrida Reuss, 1860 & Z & - & - & - & - & - & - & - & - & - & + & - & - & - & - \\
\hline Mycetophyllia horrida Reuss, 1860 & Z & - & - & - & - & - & - & - & - & - & - & - & + & - & - \\
\hline
\end{tabular}




\begin{tabular}{|c|c|c|c|c|c|c|c|c|c|c|c|c|c|c|c|}
\hline \multirow[b]{2}{*}{ Coraux scléractiniaires du Badénien de Paratéthys } & \multirow[b]{2}{*}{ 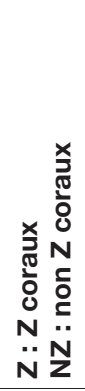 } & \multicolumn{5}{|c|}{ Lăpugiu } & \multicolumn{3}{|c|}{$\begin{array}{l}\text { Autres } \\
\text { gisements } \\
\text { roumains }\end{array}$} & \multicolumn{6}{|c|}{ Ensemble Paratéthys } \\
\hline & & 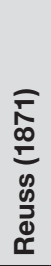 & 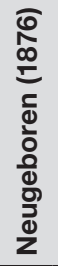 & 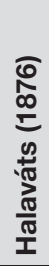 & 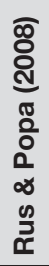 & 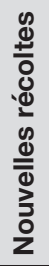 & 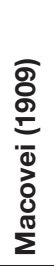 & 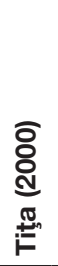 & 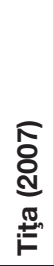 & $\begin{array}{l}\text { 동 } \\
\text { 응 } \\
0\end{array}$ & $\begin{array}{l}\frac{0}{c} \\
\frac{0}{0} \\
\frac{2}{3} \\
\frac{1}{4}\end{array}$ & $\begin{array}{l}\frac{0}{J} \\
\frac{0}{0} \\
\frac{0}{0} \\
\end{array}$ & $\begin{array}{l}\stackrel{0}{\frac{0}{5}} \\
\text { 동 } \\
\text { 오 }\end{array}$ & 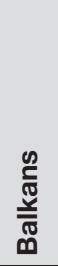 & $\begin{array}{l}. \frac{0}{\frac{3}{7}} \\
\frac{0}{5} \\
\frac{0}{3}\end{array}$ \\
\hline Syzygophyllia brevis Reuss, 1860 & Z & + & + & + & - & - & + & - & + & + & - & - & + & - & + \\
\hline Syzygophyllia crenaticostata (Reuss, 1869) & Z & - & - & - & - & + & - & - & - & - & - & - & - & - & - \\
\hline Syzygophyllia grandis Chevalier, 1962 & Z & - & - & - & - & + & - & - & - & - & - & - & - & - & - \\
\hline Mussismilia vindobonensis Chevalier, 1962 & Z & - & - & - & - & - & - & - & - & - & - & - & + & - & - \\
\hline Deltocyathus italicus (Michelotti, 1838) & $\mathrm{NZ}$ & - & - & - & - & - & + & - & - & - & - & - & - & - & - \\
\hline Deltocyathus conicus Zibrowius, 1980 & $\mathrm{NZ}$ & - & - & - & + & - & - & - & - & - & - & - & - & - & - \\
\hline Caryophyllia (Acanthocyathus) laterocristatus (M. Edw. \& H., 1848) & $\mathrm{NZ}$ & + & + & + & + & - & + & + & - & - & + & - & - & - & - \\
\hline Caryophyllia (Acanthocyathus) verrucosus M. Edw. \& H., 1848 & $\mathrm{NZ}$ & - & - & - & - & - & - & - & - & - & + & - & - & - & + \\
\hline Caryophyllia emaciata Reuss, 1871 & $\mathrm{NZ}$ & - & - & - & - & - & - & - & - & - & + & - & - & - & - \\
\hline Caryophyllia attenuata Reuss, 1871 & $\mathrm{NZ}$ & - & - & - & - & - & - & - & - & - & - & - & - & - & + \\
\hline Caryophyllia degenerans Reuss, 1871 & NZ & - & - & - & - & - & - & - & - & - & - & - & - & - & + \\
\hline Caryophyllia cladaxis Reuss, 1871 & $\mathrm{NZ}$ & - & - & - & - & - & - & - & - & - & - & - & - & - & + \\
\hline Caryophyllia truncata Reuss, 1871 & $\mathrm{NZ}$ & - & - & - & - & - & - & - & - & - & + & - & - & - & - \\
\hline Caryophyllia miocaenica Prochazka, 1893 & NZ & - & - & - & - & - & - & - & - & - & + & - & - & - & - \\
\hline Caryophyllia soosensis Prochazka, 1893 & NZ & - & - & - & - & - & - & - & - & - & + & - & - & - & - \\
\hline Caryophyllia sp. & $\mathrm{NZ}$ & - & - & - & - & - & - & - & - & + & - & - & - & - & - \\
\hline Coenocyathus depauperatus Reuss, 1871 & $\mathrm{NZ}$ & + & + & - & - & - & - & - & - & - & - & - & - & - & - \\
\hline Trochocyathus affinis Reuss, 1871 & NZ & + & + & - & - & - & - & - & - & - & + & - & - & - & - \\
\hline Trochocyathus fuchsi Reuss, 1871 & NZ & - & - & - & - & - & - & - & - & - & + & - & - & - & - \\
\hline Paracyathus firmus (Philippi, 1843) & $\mathrm{NZ}$ & + & + & - & - & - & - & - & - & - & - & - & - & - & - \\
\hline Paracyathus cupula Reuss, 1871 & $\mathrm{NZ}$ & - & - & - & - & - & - & - & - & + & - & - & - & - & - \\
\hline Paracyathus sp. & $\mathrm{NZ}$ & - & - & - & - & - & - & - & - & + & - & - & - & - & - \\
\hline Tethocyathus velatu & $\mathrm{NZ}$ & - & - & - & - & - & - & - & - & + & - & - & - & - & - \\
\hline Tethocyathus micro & NZ & - & - & - & - & - & - & - & - & + & - & - & - & - & - \\
\hline Tethocyathus walbersdorfensis Prochazka, 1893 & $\mathrm{NZ}$ & - & - & - & - & - & - & - & - & - & + & - & - & - & - \\
\hline Ceratotrochus (Ceratotrochus) multispinosus (Mich & $\mathrm{NZ}$ & + & + & - & - & - & - & - & - & - & + & - & - & - & - \\
\hline Ceratotrochus (Ceratotrochus) multiserialis (Michelotti, 1838) & NZ & + & + & - & - & - & - & - & - & - & + & - & - & - & + \\
\hline $\begin{array}{l}\text { Ceratotrochus (Edwardsotrochus) duodecimcostatus } \\
\text { (Goldfuss, 1826) }\end{array}$ & $\mathrm{NZ}$ & - & - & - & - & - & - & - & - & + & + & - & - & - & - \\
\hline Ceratotrochus (Conotrochus) typus Seguenza, 1864 & $\mathrm{NZ}$ & + & + & - & - & - & - & - & - & - & - & - & - & - & - \\
\hline Ceratotrochus granulatus Deminska-Rozkowska, 1932 & $\mathrm{NZ}$ & - & - & - & - & - & - & - & - & + & - & - & - & - & - \\
\hline Cylindrophyllia duncani (Reuss, 1871) & NZ & + & + & - & - & - & - & - & - & + & + & - & - & - & - \\
\hline Flabellum roissyanum Edwards \& Haime, 1848 & $\mathrm{NZ}$ & - & - & - & - & - & - & - & - & + & + & - & - & - & + \\
\hline Flabellum messsanense Seguenza, 1864 & $\mathrm{NZ}$ & - & - & - & - & - & - & + & - & - & - & - & - & - & - \\
\hline Flabellum multicostatum Reuss, 1871 & $\mathrm{NZ}$ & + & + & - & - & - & - & - & - & - & - & - & - & - & - \\
\hline Flabellum clavaeformis Prochazka, 1893 & NZ & - & - & - & - & - & - & - & - & - & + & - & - & - & - \\
\hline Flabellum austriacum Prochazka, 1893 & NZ & - & - & - & - & - & - & - & - & - & + & - & - & - & - \\
\hline Flabellum reussi Prochazka, 1893 & $\mathrm{NZ}$ & - & - & - & - & - & - & - & - & - & - & - & - & - & - \\
\hline Flabellum vindobonensis (Abel, 1897) & $\mathrm{NZ}$ & - & - & - & - & - & - & - & - & - & + & - & - & - & - \\
\hline Flabellum striatum Keferstein, 1859 & NZ & - & - & - & - & - & - & - & - & - & - & - & - & - & + \\
\hline Flabellum krejcii Kuhn, 1963 & $\mathrm{NZ}$ & - & - & - & - & - & - & - & - & - & - & - & - & - & - \\
\hline Flabellum sp. & $\mathrm{NZ}$ & - & - & - & - & + & - & - & - & - & - & - & - & - & - \\
\hline Porites arenosa (Esper, 1 & Z & - & - & - & - & + & + & - & - & - & - & - & - & - & - \\
\hline Porites collegniana Michelin, 1842 & Z & - & - & - & - & - & - & - & - & + & + & - & + & - & + \\
\hline Porites pusilla Felix, 1884 & $\bar{Z}$ & - & - & - & + & + & - & - & - & - & - & - & - & - & - \\
\hline Porites vindobonarum prima Kühn in Felix, 1927 & $\bar{Z}$ & - & - & - & - & - & - & - & - & + & - & - & - & - & + \\
\hline Porites maigensis gamachotensis Chevalier, 1962 & Z & - & - & - & - & + & - & - & - & - & - & - & - & - & - \\
\hline Porites collegniana lobatosepta Chevalier, 1962 & Z & - & - & - & - & + & - & - & - & - & - & - & - & - & - \\
\hline Porites mancietensis Chevalier, 1962 & $\bar{Z}$ & - & - & - & - & + & - & - & - & - & - & - & - & - & - \\
\hline Porites sp. & $\bar{Z}$ & - & - & - & + & - & - & - & - & - & - & - & - & - & - \\
\hline Alveopora meridionalis Chevalier, 1962 & Z & - & - & - & + & + & - & - & - & - & - & - & - & - & - \\
\hline Alveopora sp. & Z & - & - & - & + & - & - & - & - & - & - & - & - & - & - \\
\hline Balanophyllia (Balanophyllia) varians Reuss, 1854 & NZ & + & + & - & - & + & - & - & - & - & - & - & - & - & - \\
\hline Balanophyllia (Balanophyllia) irregularis Seguenza, 1864 & $\mathrm{NZ}$ & + & + & - & - & - & - & - & - & - & - & - & - & - & - \\
\hline Balanophyllia (Balanophyllia) concinna Reuss, 1871 & $\mathrm{NZ}$ & + & + & - & - & - & - & - & - & - & - & - & - & - & - \\
\hline Balanophyllia (Eupsammia) praelonga (Michelotti, 1838) & NZ & - & - & - & - & - & - & - & - & + & - & - & - & - & - \\
\hline Turbinaria cyathiformis (de Blainville, 1830) & Z & - & - & - & - & - & - & - & - & - & - & - & + & - & + \\
\hline Turbinaria sp. & Z & - & - & - & + & - & - & - & - & - & - & - & - & - & - \\
\hline cf. Astroides subirregularis (Osasco, 1897) & Z & - & - & - & - & - & - & - & - & - & - & - & + & - & - \\
\hline Araeacis auvertiaca (Michelin, 1844) & Z & - & - & - & + & - & - & - & - & - & - & - & - & - & - \\
\hline
\end{tabular}


calices renferment deux cycles complets de septes, de taille égale, et leur diamètre est de $1 \mathrm{~mm}$. Les septes, tous de taille égale, distinguent cette espèce de toutes celles qui renferment deux cycles complets de septes.

\section{DisCusSION}

Rus \& Popa (2008) présentent un exemplaire de Stylophora rapporté à Stylophora subreticulata Reuss, 1871 ; celui-ci possède deux cycles incomplets de septes (entre six et 12); or, l'échantillon figuré par ces auteurs ne possède que six septes par calice; comme son diamètre calicinal est de $1 \mathrm{~mm}$ (pour $S$. subreticulata, il est de $1,5 \mathrm{~mm}$ ), il se rapproche davantage de Stylophora depauperata (Reuss, 1867) du Miocène d'Indonésie.

\section{Famille SidERASTREIDAE Vaughan \& Wells, 1943}

Genre Siderastrea de Blainville, 1830

EsPÈCE TYPE. - Madrepora radians Pallas, 1766.

\section{DESCRIPTION}

Genre colonial à colonies surtout globuleuses, cérioïdes, pardfois thamnastérioïdes (sous-genre Siderofungia), à squelette plus ou moins poreux et columelle constituée d'une seule papille. Muraille synapticulothécale.

\section{Siderastrea radians (Pallas, 1766)}

(Fig. 5B)

Madrepora radians Pallas, 1766: 322.

Siderastraea radians - Cahuzac \& Chaix 1996: 113, 117, 121. Chaix \& Saint Martin 2008: 194 (avec une liste synonymique plus exhaustive).

MATÉRIEL. - Deux exemplairesde Lăpugiu, spécimen figuré: MNHN.F.A59908.

RÉPARTITION STRATIGRAPHiQUE ET GÉOGRAPHIQUE. — Oligocène supérieur: France (Cahuzac \& Chaix 1996).

Miocène inférieur: France (Cahuzac \& Chaix 1996), Mexique (Frost \& Langenheim 1974), Caraïbes (Zlatarski \& Martinez Estalella 1982).

Miocène moyen: France (Cahuzac \& Chaix 1996), Caraïbes (Zlatarski \& Martinez Estalella 1982), Crète (Chaix \& Saint Martin 2008); Roumanie (ce travail).

Miocène supérieur: Caraïbes (Zlatarski \& Martinez Estalella 1982), plate-formes carbonatées méditerranéennes (Chaix \& Saint Martin 2008).

Pliocène: Caraïbes (Zlatarski \& Martinez Estalella 1982), Vénézuela (Weisbord 1968).

Pléistocène: Caraïbes, Colombie, Vénézuela (Zlatarski \& Martinez Estalella 1982), Mauritanie (Chevalier \& Hébrard 1972), Cap-Vert et Angola (Chevalier 1970).

Actuel : Atlantique tropical ouest (Zlatarski \& Martinez Estalella 1982) et est (Chevalier 1966, 1970).

\section{DESCRIPTION}

De par ses caractères, cette espèce appartient bien au genre Siderastrea. On dénombre dans nos exemplaires entre 24 et
48 septes par calice, et le diamètre calicinal excède rarement $4,5 \mathrm{~mm}$; il n'existe pas dans cette espèce de calices polycentriques. Ce sont les caractères de $S$. radians.

\section{Siderastrea siderea (Ellis \& Solander, 1786)}

(Fig. 5C)

Madrepora siderea Ellis \& Solander, 1786: 168, tableau 49, fig. 2. Siderastrea sidereal-Milne-Edwards \& Haime 1849, t. XII: 141.

Siderastraea siderea - de Blainville sensu Pourtalès 1871: 81. - Cahuzac \& Chaix 1996: 113.

MATÉRIEL. - Deux exemplaires de Lăpugiu, spécimen figuré : MNHN.F.A59909.

RÉPARTITION STRATIGRAPHIQUE ET GÉOGRAPHIQUE. — Oligocène supérieur: France (Cahuzac \& Chaix 1996).

Miocène moyen: Jamaïque, Saint Domingue, Cuba (Vaughan1919), Roumanie (Lăpugiu, ce travail).

Pléistocène: Panama, Costa-Rica, Antilles (Vaughan 1919).

Actuel: Amérique Centrale et Sud-Est des USA et Antilles (Vaughan 1919).

\section{RAPPORTS ET DIFFÉRENCES}

Espèce voisine de $S$. radians, mais les deux exemplaires étudiés présentent des différences nettes, notamment quatre cycles complets (voire cinq incomplets) de costo-septes (entre 48 et 60) alors que S.radians n'a jamais quatre cycles complets développés. Nos exemplaires sont en nombre trop faible pour pouvoir effectuer une comparaison statistique entre les deux espèces. Le diamètre calicinal est ici de $6 \mathrm{~mm}$, alors qu'il n'atteint que $4,5 \mathrm{~mm}$ chez $S$. radians.

Rus \& Popa (2008) attribuent leurs Siderastrea à S. fröhlichiana (Reuss, 1847); ce dernier présente 48 septes, et un diamètre calicinal de $7 \mathrm{~mm}$; or l'échantillon décrit possède un peu plus de trois cycles de septes (un peu plus de 24), et un diamètre de $3 \mathrm{~mm}$; il se rapproche donc beaucoup plus de $S$. felixi Roszkowska, 1932, espèce décrite du Miocène moyen de Pologne.

Famille FAVIIDAE Gregory, 1900

Genre Favites Link, 1807

ESPÈCE TYPE. — Favites astrinus Link, 1807.

\section{DESCRIPTION}

Genre colonial, à colonies massives, cérioïdes, à petite columelle spongieuse, sans pali. Élements squelettiques non poreux. Muraille septothécale.

\section{Favites neglecta (Michelotti in d'Achiardi, 1868)} (Fig. 5D)

Aphrastraea? neglecta Michelotti in d'Achiardi, 1868: 13, pl. I, fig. 10-11. 

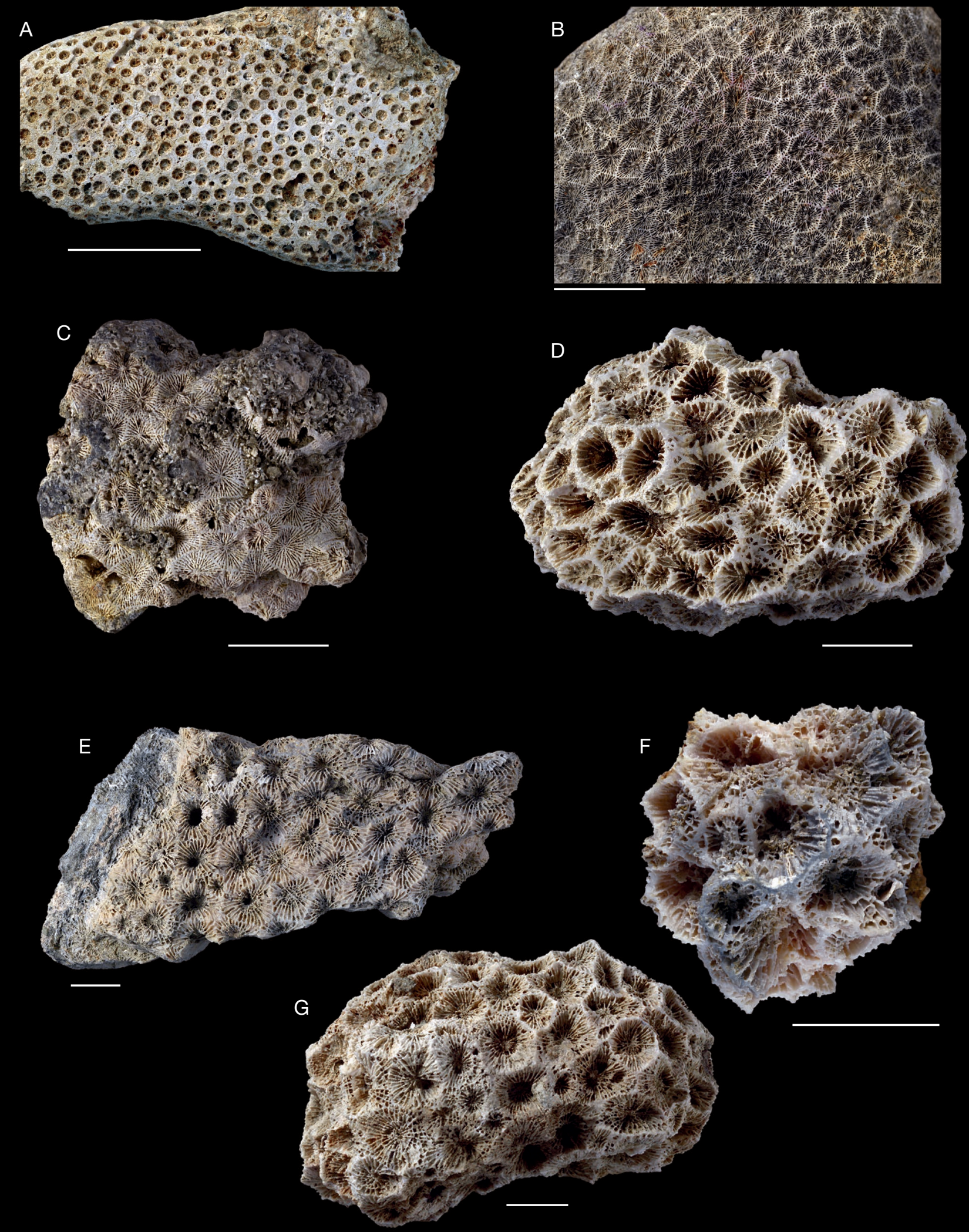

FIG. 5. - A, Stylophora calcinata (Mayer, 1864), MNHN.F.A59907; B, Siderastraea radians (Pallas, 1766), MNHN.F.A59908; C, Siderastraea siderea (Ellis \& Solander, 1786), MNHN.F.A59909; D, Favites neglecta (Michelotti in d'Achiardi, 1868), MNHN.F.A59910; E, Favites detecta (Michelotti in Sismonda, 1871), MNHN.F.A59911; F, Favites neugeboreni (Reuss, 1871), MNHN.F.A59912 ; G, Favites cf. oligocenica Chevalier, 1955, MNHN.F.A59913. Échelles: 1 cm. 
Favites neglecta-Chevalier 1962a: 138, pl. X, fig. 2, pl. XVII, fig. 16 et text fig. 45 a, b. - Cahuzac \& Chaix 1996: 112, 117, 119. Chaix et al. 2015: 372, fig. 3E-F.

MATÉRIEL. - Deux exemplaires de Lăpugiu, spécimen figuré : MNHN.F.A59910.

RÉPARTITION STRATIGRAPHIQUE ET GÉOGRAPHIQUE. — Espèce très répandue dans tout l'Oligo-Miocène atlantico-méditerranéen, sauf au Miocène supérieur.

Oligocène supérieur et Miocène inférieur: France (Chevalier 1962a Cahuzac \& Chaix 1996; Chaix et al. 2015), Turquie (Chevalier 1962a). Miocène moyen: collines de Turin (Michelotti 1838), France et Espagne (Chevalier 1962a), Roumanie (Lăpugiu, ce travail).

\section{DESCRIPTION}

Cette espèce possède tous les caractères du genre. Elle se distingue des espèces voisines par son nombre de septes (quatre cycles incomplets) et un diamètre calicinal pouvant atteindre $13 \mathrm{~mm}$.

\section{Favites detecta (Michelotti in Sismonda, 1871)}

(Fig. 5E)

Septastraea detecta Michelotti in Sismonda, 1871: 314, pl. VIII, fig. 6.

Favites detecta (Michelotti in Sismonda, 1871) - Cahuzac \& Chaix 1996: 112. — Rus \& Popa 2008: 326, pl. 1, fig. 4-5. — Chaix et al. 2015: 372, fig. 4A.

MATÉRIEL. - Quatre exemplaires de Lăpugiu, spécimen figuré: MNHN.F.A59911.

RÉPARTITION STRATIGRAPHIQUE ET GÉOGRAPHIQUE. - Espèce commune du Chattien au Miocène moyen dans le domaine atlanticoméditerranéen.

Oligocène supérieur: France (Cahuzac \& Chaix 1996).

Miocène inférieur: France (Cahuzac \& Chaix 1996), Corse (Chaix et al. 2015).

Miocène moyen : Italie (Sismonda 1871), Roumanie (Lăpugiu, ce travail)

\section{RAPPORTS ET DIFFÉRENCES}

Espèce qui ne se distingue de la précédente que par la plus petite taille de ses calices (10 $\mathrm{mm}$ maximum).

\section{Favites neugeboreni (Reuss, 1871)}

(Fig. 5F)

Prionastraea neugeboreni Reuss, 1871: 246, pl. X, fig. 2 - Neugeboren 1876: 50 .

Favites mimbastensis Chevalier, 1962a: 144, pl. XVII, fig. 4, tfs. 45c-47 - Cahuzac \& Chaix 1996: 119.

Favites cf. neglecta - Chevalier, 1962b : 25.

Favites oligocenica - Rus \& Popa 2008: 326, pl. 1, fig. 6.

MATÉRIEL. - Quatre exemplaires de Lăpugiu, spécimen figuré: MNHN.F.A59912.

Répartition STRATigraphiQue et GÉOgraphique. - Miocène inférieur: France (Cahuzac \& Chaix 1996).
Miocène moyen: Roumanie (dont Lăpugiu qui est la localitétype), Bulgarie (Gocev 1935; Kojumdgieva \& Strachimirov 1960). Miocène supérieur: Algérie et Maroc (Chaix \& Saint Martin 2008).

\section{RAPPORTS ET DIFFÉRENCES}

Espèce voisine des précédentes, elle s'en distingue par son nombre de septes (environ 30, soit quatre cycles incomplets) et des calices de $8,5 \mathrm{~mm}$ environ.

Favites cf. oligocenica Chevalier, 1955

(Fig. 5G)

Favites oligocenica Chevalier, 1955: 197, pl. VIII, fig. 3.

MatéRIEL. - Deux exemplaires de Lăpugiu, spécimen figuré: MNHN.F.A59913.

RÉPARTITION STRATIGRAPHIQUE ET GÉOGRAPHIQUE. — Oligocène inférieur: Grèce (Chevalier 1955)

Miocène moyen : Roumanie (Lăpugiu, ce travail).

\section{RAPPORTS ET DIFFÉRENCES}

Espèce très voisine de Favites detecta (Michelotti in Sismonda, 1871), elle s'en distingue par un nombre de costo-septes supérieur (44 à 50), $F$. detecta ne développant que quatre cycles incomplets $(\mathrm{n}<48)$; par ailleurs le diamètre calicinal est un peu plus grand (12 $\mathrm{mm}$ au lieu de 10$)$.

Il y a doute sur l'identification des échantillons roumains : ils sont peu nombreux, le nombre de septes est de 48 et le diamètre calicinal est de $11 \mathrm{~mm}$. Les deux espèces sont peut-être synonymes.

Genre Heliastraea Milne-Edwards \& Haime, 1857

EsPÈCE TYPE. - Heliastraea forskaeli Milne-Edwards \& Haime, 1857.

\section{DESCRIPTION}

Les Heliastraea sont des formes massives, à squelette compact, à disposition plocoïde des calices et columelle spongieuse, très bien redéfini par Chevalier (1954). Montastrea est un nomen nudum, jusqu'ici jamais défini clairement, et qui doit donc disparaître de la nomenclature (cf. Chevalier 1954: 105, 106).

\section{Sous-genre Aquitanastraea Chevalier, 1962}

ESPÈCE TYPE. - Aquitanastraea piveteaui Chevalier, 1954.

\section{DESCRIPTION}

Ce sous-genre et très voisin d'Heliastraea s. s.; il ne s'en distingue que par des microcolonnnes verticales reliant les dissépiments exothécaux. Chez Echinopora les côtes sont totalement transformées en épines et la muraille peut parfois être perforée. 
Heliastraea (Aquitanastraea) guettardi (Michelin, 1841) (Fig. 6A)

Astrea guettardi Michelin, 1841 in Michelin 1840-1847: 58, pl. 12, fig. 3 .

Heliastraea guettardi - Milne-Edwards \& Haime 1857, II: 461. Chevalier 1954: 153.

Aquitanastraea guettardi - Chevalier 1962a: 188, pl. IX, fig. 1.

MATÉRIEL. - 53 exemplaires de Lăpugiu, spécimen figuré: MNHN.F.A59914.

RÉPARTITION STRATIGRAPHIQUE ET GÉOGRAPHIQUE. - Espèce très localisée. Miocène moyen: Italie (Michelin 1840-1847) et Roumanie (Lăpugiu, ce travail).

\section{DESCRIPTION}

Comme tous les Aquitanastraea, cette espéce est masssive, plocoïde, à columelle spongieuse et costo-septes très dentés. Heliastraea (Aquitanastraea) guettardi est une espèce possédant de grands calices (diamètre $10 \mathrm{~mm}$ ) et un grand nombre d'éléments radiaires (50 à 60); ces deux caractères la distinguent de toutes les espèces voisines. La constitution de l'exothèque (nombreux dissépiments reliés par des micropiliers verticaux) l'intègre au sous-genre Aquitanastraea.

Heliastraea (Aquitanastraea) incrustans (Osasco, 1897) (Fig. 6B)

Heliastraea incrustans Osasco, 1897: 8, fig. 3.

Aquitanastraea incrustans - Chevalier 1954: 152, fig. 29, pl. 5, fig. 4; 1962a: 187.

Heliastraea (Aquitanastraea) incrustans - Cahuzac \& Chaix 1996: 112, 119, 121. - Chaix et al. 2015: 372, fig. 4B.

MATÉRIEL. - 35 exemplaires de Lăpugiu, spécimen figuré : MNHN.F.A59915.

RÉPARTITION STRATIGRAPHIQUE ET GÉOGRAPHIQUE. - Oligocène supérieur: France (Cahuzac \& Chaix 1996).

Miocène inférieur: France (Cahuzac \& Chaix 1996; Chaix et al. 2015).

Miocène moyen: France (Cahuzac \& Chaix 1996), Italie (Osasco 1897), Roumanie (Lăpugiu, ce travail).

\section{RAPPORTS ET DIFFÉRENCES}

Cette espèce, très voisine de la précédente, ne s'en distingue que par le nombre de ses éléments radiaires (quatre cycles incomplets) et son diamètre calicinal $(7 \mathrm{~mm})$.

Heliastraea (Aquitanastraea) pelouaensis (Chevalier, 1954) (Fig. 6C)

Heliastraeopsis pelouaensis Chevalier, 1954: 147, pl. VII, fig. 1.

Heliastraea (Heliastraea) pelouaensis - Cahuzac \& Chaix 1996: $117,119$.
MATÉRIEL. - Quatre exemplaires de Lăpugiu, spécimen figuré: MNHN.F.A59916.

RÉPARTITION STRATIGRAPHIQUE ET GÉOGRAPHIQUE. - Miocène inférieur: France (Chevalier 1954, 1962a; Cahuzac \& Chaix 1996). Miocène moyen: Italie (Chevalier 1954), Roumanie (Lăpugiu, ce travail).

\section{RAPPORTS ET DIFFÉRENCES}

Cette espèce, très voisine de la précédente, ne s'en distingue que par des calices un peu plus grands ( $8 \mathrm{~mm}$ au lieu de $7 \mathrm{~mm}$ ). Rappelons que toutes ces espèces d'Heliastraea s.l. sont très voisines et ne se distinguent que par les caractères mentionnés.

\section{REMARQUES}

Le changement de sous-genre proposé dans la liste synonymique est possible grâce à la bonne conservation de nos échantillons; à l'inverse, celle-ci ne permet pas toujours (pour d'autres faunes moins bien conservées), l'observation des micropiliers de l'exothèque, bien visibles sur la figuration de Chevalier (1954: 148, fig. 26).

\section{Heliastraea (Aquitanastraea) solenastroides (Chevalier, 1954)}

(Fig. 6D)

Heliastreopsis solenastroides Chevalier, 1954: 149, pl. I, fig. 6.

Heliastraea (Heliastraea) solenastroides-Cahuzac \& Chaix 1996: 119.

MATÉRIEL. — Un exemplaire de Lăpugiu (MNHN.F.A59917).

Répartition STRATIGRAPHIQUe ET GÉOGRAPHIQUe. - Miocène inférieur: France (Chevalier 1954; Cahuzac \& Chaix 1996).

Miocène moyen: Roumanie (Lăpugiu, ce travail).

\section{RAPPORTS ET DIFFÉRENCES}

Espèce encore très voisine des précédentes, mais on n'observe dans les calices que 22 costoseptes (trois cycles incomplets) alors que les calices, eux sont plutôt grands $(8 \mathrm{~mm})$.

Sous-genre Heliastraea Milne-Edwards \& Haime, 1857

ESPÈCE TYPE. — Heliastraea forskaeli Milne-Edwards \& Haime, 1857.

\section{DESCRIPTION}

Les Heliastraea (s.str.) se distinguent des Aquitanastraea par l'absence de micropiliers verticaux dans l'exothèque; de plus les côtes sont plus ou moins dissociées, et très épineuses.

Heliastraea (Heliastraea) brevis (Duncan, 1863) (Fig. 6E)

Astraea brevis Duncan, 1863: 37, pl. IV, figs 3a-3b.

Orbicella mellahica Gregory, 1906: 52, pl. VI, fig. 3-4.

Orbicella brevis - Vaughan 1919: 391, pl. 97, fig. 1. 
Non Heliastraea oligophylla major - Hegedűs 1970: 186, pl. 1, fig. 2. Heliastraea aff. mellahica-Scholz 1970: 196, pl. 3, fig. 3; pl. 4, fig. 2. Montastrea mellahica - Oosterbaan 1990: 9, pl. 1, fig. 6.

MATÉRIEL. — Un exemplaire de Lăpugiu (MNHN.F.A59918).

RÉPARTITION STRATIGRAPHIQUE ET GÉOGRAPHIQUe. - Miocène moyen: Antilles (Duncan 1863; Vaughan 1919), Égypte (Gregory 1906), Hongrie (Hegedűs 1970; Scholz 1970; Oosterbaan 1990), Roumanie (Lăpugiu, ce travail).

\section{DESCRIPTION}

L'espèce décrite ici comporte quatre cycles incomplets de septes et un diamètre calicinal de $5 \mathrm{~mm}$. Elle possède par ailleurs tous les caractères du sous-genre.

Heliastraea (Heliastraea) saubriguensis Chevalier, 1962 (Fig. 6F)

Heliastraea saubriguensis Chevalier, 1962a: 179, pl. VI, fig. 1 et tf. 60. Heliastraea (Heliastraea) saubriguensis - Cahuzac \& Chaix 1996: 121.

MATÉRIEL. - 47 exemplaires de Lăpugiu, spécimen figuré: MNHN.F.A59918.

RÉPARTITION STRATIGRAPHIQUE ET GÉOGRAPHIQUE. - Miocène moyen: France (Chevalier 1962a; Cahuzac \& Chaix 1996) et Roumanie (Lăpugiu, ce travail).

\section{RAPPORTS ET DIFFÉRENCES}

Espèce voisine de la précédente; elle s'en distingue par son nombre de septes (trois cycles complets, soit 24) et son diamètre calicinal $(6 \mathrm{~mm})$.

Genre Tarbellastraea Alloiteau, 1950

EsPÈCE TYPE. — Astrea ellisiana Defrance, 1826.

\section{DESCRIPTION}

Genre à colonie plocoïde, à petits calices circulaires, columelle lamellaire, endothèque et exothèque rares et subtabulaires.

\section{Tarbellastraea ellisiana (Defrance, 1826)} (Fig. 6G)

Astrea ellisiana Defrance, 1826: 382.

Stylina thyrsiformis Michelin, 1841 in Michelin 1840-1847: 50, 51, pl. 10, figs 6, 7.

?S. stricta Michelin, 1841 in Michelin 1840-1847: 50, 51, pl. 10, figs 6,7 .

Phyllocoenia carryana d'Orbigny, 1852: 147.

Heliastraea conoidea Reuss, 1871: 240, pl. 10, fig. 3. - Neugeboren 1876: 48.
Tarbellastraea ellisiana (pars) - Alloiteau 1957: 128, non pl. 8, figs 4, 15. - Chevalier 1962a: 194. - Cahuzac \& Chaix 1996: 117, 119, 121. — Budd et al. 1996: 546, pl. III, fig. 2 (non fig. 1 = Antiguastraea alveolaris (Catullo, 1856)).

Tarbellastraea carryensis Chevalier, 1962a: 195, pl. V, fig. 8, pl. VII, fig. 4, pl. XXIV, fig. 6. — Budd et al. 1996: 543, pl. I, figs 5, 6.

MATÉRIEL. - Un exemplaire de Lăpugiu, spécimen figuré : MNHN.F.A59920.

RÉPARTITION STRATIGRAPHIQUE ET GÉOGRAPHIQUE. - Miocène inférieur: France (Chevalier 1962a; Cahuzac \& Chaix 1996), Portugal (Chevalier \& Nascimento 1975).

Miocène moyen: France (Chevalier 1962a; Cahuzac \& Chaix 1996), Italie (Chevalier 1962a), Roumanie (Lăpugiu: Reuss 1871; Neugeboren 1876, et ce travail), Autriche et Hongrie (Reuss 1871).

Miocène supérieur: Afrique du Nord (Chaix \& Saint Martin 2008).

\section{DESCRIPTION}

Cette espèce, très abondante dans tout l'Oligo-Miocène atlantico-méditerranéen, possède quatre cycles incomplets de septes et des diamètres calicinaux allant de 2,5 à $4 \mathrm{~mm}$, tout comme Antiguastraea alveolaris (Catullo, 1856) qui est aussi plocoïde, avec une columelle lamellaire, mais dont exothèque et endothèque sont très différentes. Les mêmes différences s'observent pour Tarbellastraea reussiana (espèce étudiée plus loin) dont beaucoup d'exemplaires cités en littérature doivent être reclassés dans une autre espèce d'Antiguastraea que C. Chaix a récoltée tant dans l'Oligo-Miocène d'Aquitaine que dans le Miocène de Méditerranée.

Tarbellastraea reussiana (Milne-Edwards \& Haime, 1850) (Fig. 6H)

Astraea reussiana Milne-Edwards \& Haime, 1850, pars 4: 110.

Astraea astroites (pars?) - de Blainville 1834: 269.

Explanaria astroites Reuss, 1847: 17, pl. II, figs 7, 8.

Explanaria tenera (pars?) Reuss, 1847: 18, pl. III, fig. 2.

Astraea moravica (pars?) Reuss, 1847: 23, pl. IV, fig. 4.

Astraea raulini Milne-Edwards \& Haime, 1850, pars 4: 110.

Astraea prevostiana Milne-Edwards \& Haime, 1850, pars 4: 110.

Solenastraea manipulata Reuss, 1871: 243, pl. VIII, fig. 2.

Heliastraea acervularia Mayer-Eymar, 1883: 73, pl. XXIII, fig. 1.

Orbicella eggenburgensis formosa Kühn, 1925: 7, pl. I, fig. 3.

Solenastraea tizeroutinensis Chevalier, 1962a: 190, pl. XVII, figs 2, 21.

Tarbellastraea eggenburgensis andalousianensis Chevalier, 1962a: 203, pl. IX, fig. 13, pl. XXIII, fig. 9.

Tarbellastraea abditaxis Chevalier, 1962a: 204, pl. VI, fig. 10, pl. XXIII, fig. 8, text-fig. 66.

Tarbellastraea siciliae Chevalier, 1962a: 207, pl. VII, fig. 10, pl. XXIII, fig. 7. 

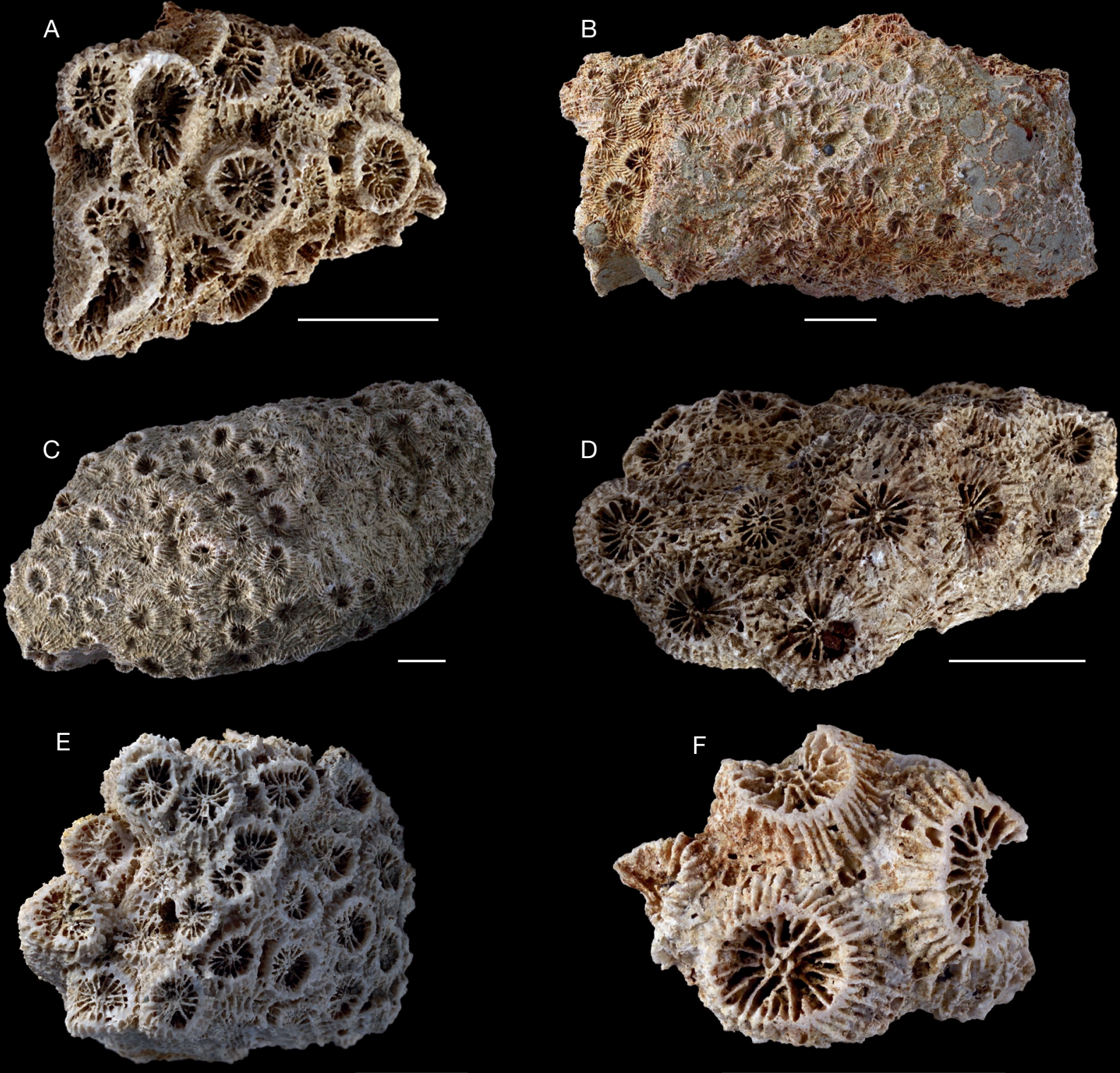

$\mathrm{H}$
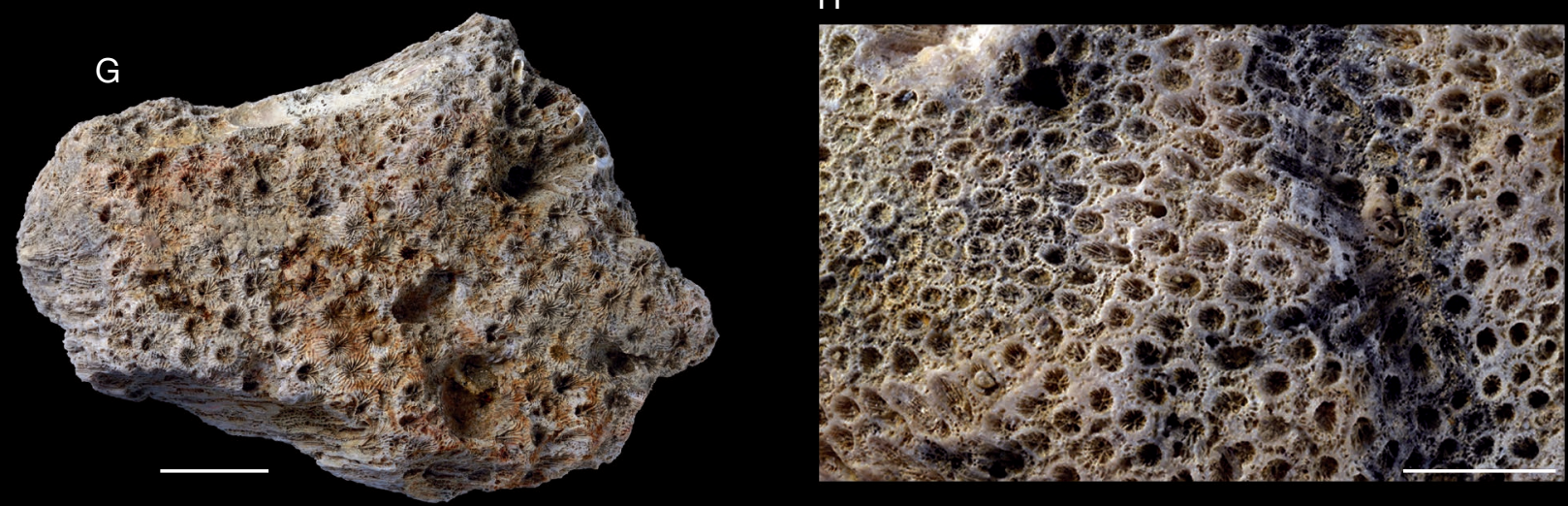

FIG. 6. - A, Heliastraea (Aquitanastraea) guettardi (Michelin, 1841), MNHN.F.A59914 ; B, Heliastraea (Aquitanastraea) incrustans (Osasco, 1897), MNHN.F.A59915; C, Heliastraea (Aquitanastraea) pelouaensis (Chevalier, 1954), MNHN.F.A59916; D, Heliastraea (Aquitanastraea) solenastroides (Chevalier, 1954), MNHN.F.A59917; E, Heliastraea (Heliastraea) brevis (Duncan, 1863), MNHN.F.A59918; F, Heliastraea (Heliastraea) saubriguensis Chevalier, 1962, MNHN.F.A59919; G, Tarbellastraea ellisiana (Defrance, 1826), MNHN.F.A59920; H, Tarbellastraea reussiana (Milne-Edwards \& Haime, 1850), MNHN.F.A59921. Échelles: $1 \mathrm{~cm}$. 
Paleoplesiastraea romettensis Chevalier, 1962a: 266.

Palaeoplesiastrea batteriae Chevalier, 1962a: 270, pl. X, fig. 6, pl. XXIV, fig. 8, text-fig. 99.

?Solenastraea cf. peroni Chevalier, 1962b: 27, pl. IV, fig. 2.

Tarbellastraea cf. conoidea Chevalier, 1962b: 29.

Paleoplesiastraea columnaeformis montjamonti Chevalier, 1962b: 33, pl. II, fig. 2, pl. III, fig. 14, pl. IV, fig. 4.

?Tarbellastraea sp. - Saint Martin \& Cornée 1996: 235, tableau 1.

Non Tarbellastraea abditaxis Budd, Bosellini \& Stemann 1996: 540, pl. I, figs 1, 2.

Tarbellastraea bragai Budd, Bosellini \& Stemann 1996: 542, pl. I, figs 3, 4 .

Heliastraea reussiana - Milne-Edwards \& Haime 1857: 474.

Heliastraea raulini-Milne-Edwards \& Haime 1857: 474.

Heliastraea prevostiana - Milne-Edwards \& Haime 1857: 475.

Orbicella reussiana (pars?) - Kühn 1925: 67.

Tarbellastraea raulini - Chevalier 1962a: 200, pl. XIII, fig. 3, textfig. 63a. — Budd et al. 1996: 550, pl. IV, figs 1-4.

Tarbellastraea reussiana (pars) - Chevalier 1962a: 205, pl. X, fig. 1, pl. XXIV, fig. 4.

Tarbellastraea reussiana echinulata - Chevalier 1962a: 206, pl. V, fig. 18, pl. XXIV, fig. 7.

Tarbellastraea prevostiana - Chevalier 1962a: 206.

Tarbellastraea reussiana - Chevalier 1962b: 28, pl. 2, fig. 1. - Chevalier in Rangheard 1969: 287. — Chaix et al. 1986: 220. - Saint Martin 1990: nombreuses citations. - Cahuzac \& Chaix 1996 117, 119, 121. — Budd et al. 1996: 550, pl. IV, figs 5, 6.

Tarbellastraea cf. reussiana - Saint Martin 1996: 242, 244, tableau 1.

Tarbellastraea tenera - Budd et al. 1996: 553, pl. II, figs 3-5.

Dans cette liste, plusieurs auteurs ont cité l'espèce Tarbellastraea reussiana pour Lăpugiu, sous les noms:

Explanaria astroites Reuss, 1847: 17, pl. II, figs 7-8.

Solenastrea manipulata Reuss, 1871: 243, pl. VIII, fig. 2.

Heliastraea Reussana - Neugeboren 1876: 48.

MATÉRIEL. - 77 exemplaires de Lăpugiu, spécimen figuré : MNHN.F.A59921.

RÉPARTITION STRATIGRAPHIQUE ET GÉOGRAPHIQUE. - Même répartition que pour Tarbellastraea ellisiana.

\section{DESCRIPTION}

En ce qui concerne les caractères propres à cette espèce, nous avons pu établir, à partir de nombreuses colonies du bassin de la Messara en Crète, que la variabilité de cette espèce est extrêmement grande: sur un seul fragment de colonie, les calices (adultes uniquement, possédant 24 septes) présentent des diamètres compris entre 0,9 et $3,5 \mathrm{~mm}$, et des distances centre-à-centre comprises entre 1,5 et $4 \mathrm{~mm}$. Cette variabil- ité, au sein d'une même colonie, a été observée sur tous les spécimens. Il en résulte que tous les Tarbellastraea possédant trois cycles complets de septes (soit 24), des diamètres calicinaux compris entre 0,9 et $3,5 \mathrm{~mm}$ (tous mesurés sur calices adultes) et des distances centre-à-centre comprises entre 1,5 et $4 \mathrm{~mm}$ appartiennent à la même espèce, soit Tarbellastraea reussiana, ce qui se traduit par la synonymie proposée plus haut. La morphologie des colonies est également extrêmement variable, elle peut être massive, lamellaire ou colonnaire, avec tous les intermédiaires possibles, en fonction des conditions de milieu locales. Ce fait confirme, si besoin en était, que l'allure des colonies, modulée par l'environnement, ne saurait être utilisée comme critère d'identification, seuls les caractères squelettiques pouvant être utilisés à cette fin.

\section{Genre Antiguastraed Vaughan, 1919}

ESPÈCE TYPE. - Astrea cellulosa Duncan, 1863.

\section{DESCRIPTION}

Genre colonial, récifal, plocoïde à petis calices, columelle plus ou moins lamellaire, éléments squelettiques compacts. Antiguastraea a été parfaitement défini par Vaughan (1919): «Growth form massive; asexual reproduct ion by intercorallite budding; septal margins very obscurely dentate, subentire; corallites usually joined by thin costae; columella lamellar, usually well developed and prominent; exothecal and endothecal dissepiments highly developed ». Les Antiguastraea, très voisins des Tarbellastraea, s'en distinguent par un squelette moins dense, et par endothèque et exothèque entièrement vésiculeuses, et surabondantes.

\section{Antiguastraea aquitaniensis (Chevalier, 1962)} (Fig. 7A)

Tarbellastraea aquitaniensis Chevalier, 1962a - 201, pl. 9 , fig. 12 et pl. 23, fig. 2.

Non Solenastraea tenera Reuss, 1871: 242, pl. 7, fig. 5. - Neugeboren 1876: 49 .

Antiguastraea sp. 1 - Cahuzac \& Chaix 1996: 117, 119, 121.

MATÉRIEL. - Nombreux exemplaires de Lăpugiu, spécimen figuré: MNHN.F.A59922.

Répartition STRatigraphiQue ET GÉOgRaPhiQue. - Miocène inférieur: France (Chevalier 1962a; Cahuzac \& Chaix 1996).

Miocène moyen: France (Cahuzac \& Chaix 1996); Italie (Chevalier 1962a) et Roumanie, Lăpugiu (Reuss 1871 ; Neugeboren 1876).

\section{DESCRIPTION}

Antiguastraea aquitaniensis possède trois cycles complets de septes (24) et un diamètre calicinal de $2 \mathrm{~mm}$. Tous les autres caractères sont ceux du genre Antiguastraea.

DisCUSSION

Toutes les espèces rattachées soit à Antiguastraea, soit à Tarbellastraea, souffrent d'une ambiguité majeure: la confusion 
effectuée par tous les auteurs, depuis la création du genre Tarbellastraea par Alloiteau (1957), avec le genre Antiguastraea qui pourtant a été parfaitement défini par Vaughan en 1919 (voir plus haut). Il est donc clair (notamment au vu des figures 3-3a, 4-4a de la planche 98 de Vaughan) que les seules différences entre Tarbellastraea et Antiguastraea résident dans la nature de l'endothèque et de l'exothèque (et non dans l'abondance de ces éléments, qui est un facteur dépendant du milieu) : endothèque et exothèque rares et tabulaires chez Tarbellastraea, surabondantes et vésiculeuses chez Antiguastraea. Les récoltes abondantes sur le terrain confirment l'existence de deux stocks correspondant précisément aux deux genres en question: un premier stock possède un squelette très peu dense, avec une endothèque et une exothèque uniquement vésiculeuses, par ailleurs très abondantes: c'est la définition même du genre Antiguastraea; l'autre stock possède un squelette relativement dense, dont l'endothèque et l'exothèque sont rares et uniquement tabulaires: il faut réserver à ce stock la dénomination de Tarbellastraea. C'est ainsi que tous les spécimens dénommés jusqu'ici Tarbellastraea ellisiana, possédant quatre cycles incomplets de septes et des diamètres calicinaux allant de 2,5 à $4 \mathrm{~mm}$, doivent être partagés entre cette espèce et Antiguastraea alveolaris (Catullo, 1856) qui est aussi plocoïde, avec une columelle lamellaire, mais dont exothèque et endothèque ont les propriétés décrites ci-dessus. Il en est de même pour Tarbellastraea reussiana dont beaucoup d'exemplaires cités en littérature doivent être reclassés dans une autre espèce d'Antiguastraea que C. Chaix a récoltée tant dans l'Oligo-Miocène d'Aquitaine que dans le Miocène de Méditerranée. Toutes les études sur Antiguastraea et Tarbellastraea sont à reprendre sur ces bases, les deux genres étant aisés à distinguer sur le terrain, et cohabitant parfois.

Genre Plesiastraea Milne-Edwards \& Haime, 1848

ESPĖCE TYPE. - Astrea versipora Lamarck, 116.

\section{DESCRIPTION}

Genre colonial, plocoïde, récifal, à petits calices, columelle papilleuse et couronne de pali devant les S3.

Sous-genre Paleoplesiastraea Chevalier, 1962

ESPÈCE TYPE. — Plesidstraea desmoulinsi Milne-Edwards \& Haime, 1851.

\section{DESCRIPTION}

Ce sous-genre difere de Plesiastraea s.s. par une granulation costale totalement irrégulière, alors qu'elle est disposée en une seule rangée hez Plesiastraea.

\section{Plesiastraea (Paleoplesiastraea) desmoulinsi}

(Milne-Edwards \& Haime, 1851)

(Fig. 7B)

Plesiastraea desmoulinsi Milne-Edwards \& Haime, 1851b: 100.
Stylina inopinata Reuss, 1871: 237, pl. 7, fig. 3.

Palaeoplesiastraea desmoulinsi-Chevalier 1962a: 264, pl. XIII, fig. 4, pl. XXIV, fig. 5, text-fig. 96, 97.

Plesiastraea (Palaeoplesiastraea) desmoulinsi-Cahuzac \& Chaix 1996: 112, 117, 119. - Chaix \& Saint Martin 2008: 185, fig. 2B. Chaix et al. 2015: 376 .

MATÉRIEL. - Trois exemplaires de Lăpugiu, spécimen figuré: MNHN.F.A59923.

RÉPARTITION STRATIGRAPHIQUE ET GÉOGRAPHIQUE. - Oligocène supérieur: France (Cahuzac \& Chaix 1996).

Miocène inférieur: France (Cahuzac \& Chaix 1996; Chaix et al. 2015), Iran (matériel Ghaedi non publié).

Miocène moyen: France (Cahuzac \& Chaix 1996), Espagne (Chevalier 1962a), Italie (Chevalier 1962a), Hongrie et Autriche (Reuss 1871), Pologne (Roszkowska 1932), Roumanie (Lăpugiu, ce travail). Miocène supérieur de Méditerranée (Chaix \& Saint Martin 2008).

\section{DESCRIPTION}

Plesiastraea $(P$.) desmoulinsi possède trois cycles complets de septes, des côtes bien développées, des pali larges, et un diamètre calicinal de 4 à $5 \mathrm{~mm}$. Les autres caractères sont ceux du sous-genre.

\section{Plesiastraea (Palaeoplesiastraea) tazarinensis \\ (Chevalier, 1962) \\ (Fig. 7C)}

Cyphastraea (?) tazarinensis Chevalier, 1962a: 263, pl. XI, fig. 4.

Non Cyphastraea coloi Chevalier, 1962a: 262, pl. XI, fig. 12

Plesiastraea (Palaeoplesiastraea) tazarinensis - Cahuzac \& Chaix 1996: 117, 119.

Plesiastraea (Palaeoplesiastraea) coloi-Chaix \& Saint Martin 2008: 186, fig. 2C.

MATÉRIEL. - Deux exemplaires de Lăpugiu, spécimen figuré : MNHN.F.A59924.

Répartition Stratigraphique et Géographique. - Miocène inférieur: France (Cahuzac \& Chaix 1996).

Miocène moyen : Roumanie (Lăpugiu, ce travail).

Miocène supérieur: Méditerranée (Chaix \& Saint Martin 2008).

\section{RAPPORTS ET DIFFÉRENCES}

Cette espèce se distingue de la précédente par des calices beaucoup plus petits ( $2 \mathrm{~mm}$ au lieu de 4$)$ et des pali plus petits.

\section{Famille AstrangIIDAE Verrill, 1870}

Genre Cladangia Milne-Edwards \& Haime, 1851

ESPÈCE TYPE. - Astrea semispherica Defrance, 1826.

\section{DESCRIPTION}

Genre colonial et récifal, plocoïde, à calices assez grands, columelle papilleuse et sans pali. 
Cladangia conferta (Reuss, 1847)

(Fig. 7D)

Cladocora conferta Reuss, 1847: 19, pl. III, figs 4-5.

Cladangia conferta - Reuss 1871: 247, pl. 16, fig. 1-7, pl. 18, fig. 3.

MatérIel. - Un exemplaire de Lăpugiu, spécimen figuré: MNHN.F.A59925.

RÉPARTITION STRATIGRAPHIQUE ET GÉOGRAPHIQUE. - Cette espèce semble localisée au Miocène moyen de la Paratéthys: Autriche, République tchèque (Reuss 1871). Elle est citée ici pour la première fois à Lăpugiu.

\section{DESCRIPTION}

Cladangia conferta, à côté de caractères typiques du genre, possède quatre cycles incomplets de septes, et un diamètre calicinal maximal de $7,5 \mathrm{~mm}$.

\section{Genre Oulangia Milne-Edwards \& Haime, 1848}

ESPÈCE TYPE. — Oulangia stokesiana Milne-Edwards \& Haime, 1850.

\section{DESCRIPTION}

Genre colonial, reptoïde, à columelle papilleuse. Le genre Oulangia Milne-Edwards \& Haime, 1848 est un corail colonial dont on ne retrouve le plus souvent que des polypiérites solitaires car le squelette qui entoure les calices (coenosteum) est assez ténu et fragile. Quand on les retrouve entières, les colonies sont très largement fixées par toute leur surface. Par ailleurs, les septes sont dentés et exserts, et la columelle est papilleuse; tous ces caractères sont identiques chez Brachytrochus Reuss, 1864.

\section{Oulangia speyeri (Reuss, 1864)}

(Fig. 7E)

Brachytrochus speyeri Reuss, 1864: 619, pl. 6, fig. 2a-b.

Oulangia speyeri - Cahuzac \& Chaix 1996: 112, 119, 121.

Matériel. - Un exemplaire de Lăpugiu, spécimen figuré: MNHN.F.A59926.

RÉPARTITION STRATIGRAPHIQUE ET GÉOGRAPHIQUE. — Oligocène supérieur: France (Cahuzac \& Chaix 1996), Allemagne (Reuss 1864). Miocène inférieur: France (Cahuzac \& Chaix 1996).

Miocène moyen: France (Cahuzac \& Chaix 1996) et Roumanie (Lăpugiu, ce travail).

\section{DESCRIPTION ET DISCUSSION}

Oulangia speyeri, en dehors des caractères génériques, se distingue des autres espèces du genre par ses quatre cycles incomplets de septes et son diamètre calicinal de $2 \mathrm{~mm}$.

Reuss (1871) et Neugeboren (1876) citent un autre Astrangiidae à Lăpugiu: Rhizangia procurrens Reuss, 1871. Les deux espèces sont très voisines, des exemplaires solitaires d'Oulangia pouvant être confondus avec des Rhizangia.
Famille OCULINIDAE Gray, 1847

Genre Astrohelia Milne-Edwards \& Haime, 1849

ESPÈCE TYPE. — Madrepora palmata Goldfuss, 1829.

\section{DESCRIPTION}

Genre colonial, récifal, de morphologie variable, plocoïde, à périthèque porcelanée et septes disposés peu ou prou irrégulièrement, à dentition septale peu visible. Columelle profonde à absente.

\section{Astrohelia gaillardensis Chevalier, 1962}

(Fig. 7F)

Astrohelia gaillardensis Chevalier, 1962a: 257, pl. VIII, fig. 15, pl. XI, fig. 13, tf. 95.

Matériel. - Cinq exemplaires de Roșcani, spécimen figuré: MNHN.F.A59927.

Répartition STRATIGRAPHiQUe ET GÉOGRAPHIQUe. - Miocène inférieur: France (Chevalier 1962a).

Miocène moyen: Roumanie (Roșcani, ce travail).

\section{RAPPORTS ET DIFFÉRENCES}

A. gaillardensis, hors des caractères génériques, se différencie des espèces voisines par ses trois cycles complets de septes (24) et son diamètre calicinal de $2,5 \mathrm{~mm}$.

\section{Famille Mussidae Ortmann, 1890}

Genre Lithophyllia Milne-Edwards \& Haime, 1857

ESPÈCE TYPE. - Madrepora lacera Pallas, 1766.

\section{DESCRIPTION}

Genre de corail solitaire, vivant surtout en récif, à base patelloïde puis croissance cylindrique, à squelette fortement denté, à muraille parathécale et columelle spongieuse, sans pali.

\section{Lithophyllia coronula (Michelotti, 1838)}

$$
\text { (Fig. 7G) }
$$

Fungia coronula Michelotti, 1838: 94.

Anthophyllum detritum Michelin, 1841 in Michelin 1840-1847: 48, pl. 10, fig. 1.

Montlivaultia patula Michelotti in Sismonda, 1871: 338, pl. VII, fig. 4.

Lithophyllia patula-Chevalier 1962a: 278, text-fig. 100b. - Cahuzac \& Chaix 1996: 112, 117, 119, 121.

MATÉRIEL. - Trois exemplaires de Lăpugiu, spécimen figuré : MNHN.F.A59928. 


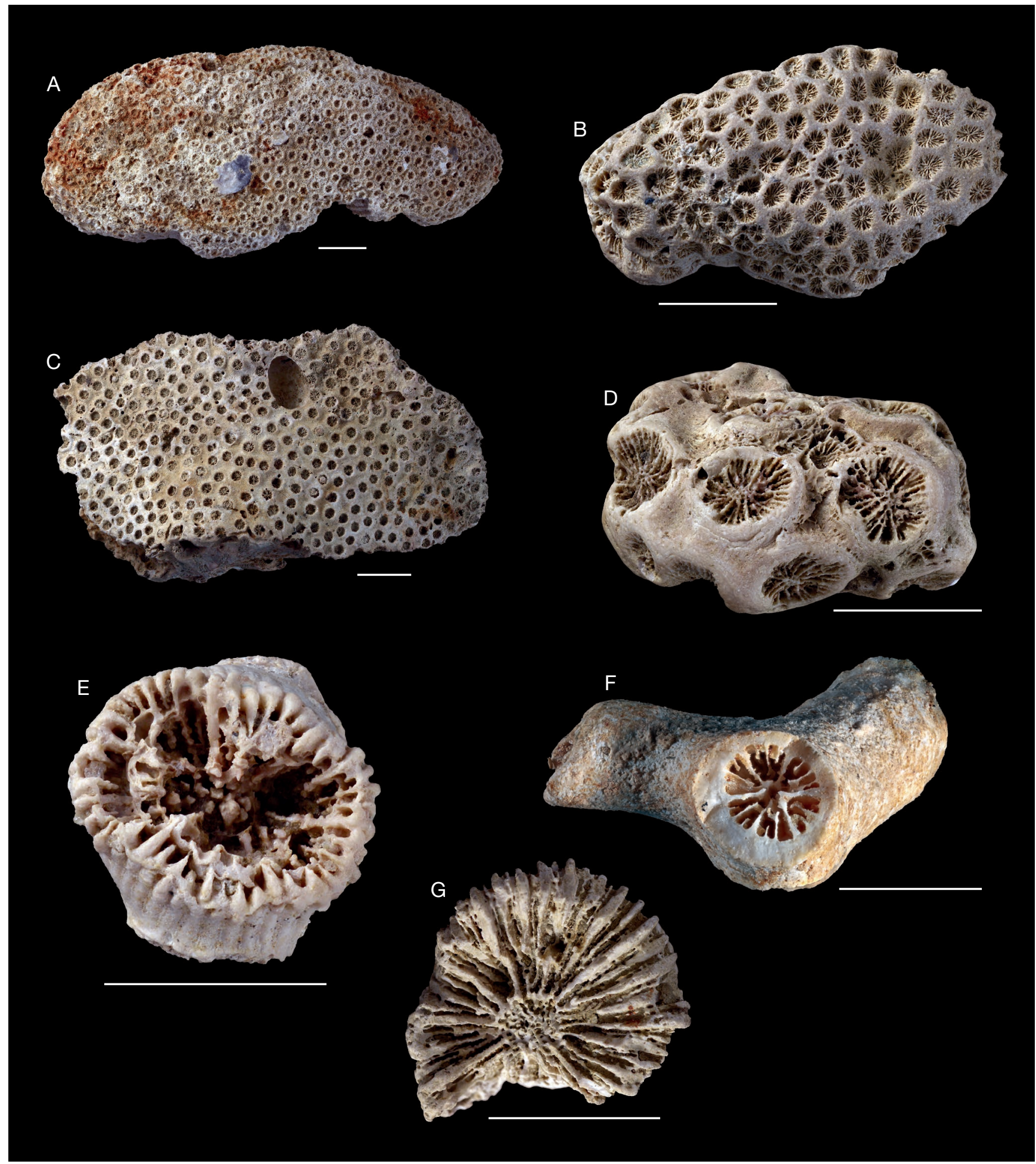

FiG. 7. - A, Antiguastraea aquitaniensis (Chevalier, 1962a), MNHN.F.A59922; B, Plesiastraea (Paleoplesiastraea) desmoulinsi (Milne-Edwards \& Haime, 1851), MNHN.F.A59923; C, Plesiastraea (Palaeoplesiastraea) tazarinensis (Chevalier, 1962), MNHN.F.A59924; D, Cladangia conferta (Reuss, 1847), MNHN.F.A59925; E, Oulangia speyeri (Reuss, 1864), MNHN.F.A59926; F, Astrohelia gaillardensis Chevalier, 1962, MNHN.F.A59927; G, Lithophyllia coronula (Michelotti, 1838) MNHN.F.A59928. Échelles: A-D, 1 cm; E, F, 0,5 cm.

RÉPARTITION STRATIGRAPHIQUE ET GÉOGRAPHIQUE. — Oligocène supérieur: France (Cahuzac \& Chaix 1996), Italie (Michelotti 1838). Miocène inférieur: France (Cahuzac \& Chaix 1996).

Miocène moyen : France (Cahuzac \& Chaix 1996), Italie (Michelotti 1838 ; Chevalier 1962a), Roumanie (Lăpugiu, ce travail).

\section{DESCRIPTION}

L'espèce figurée ici, qui possède tous les caractères de Lithophyllia, se distingue des autres par son nombre de septes (cinq cycles incomplets, soit un nombre compris entre 48 et 96 septes), et son diamètre calicinal (35 $\mathrm{mm}$ au maximum). 
Genre Acanthophyllia Wells, 1937

ESPÈCE TYPE. - Caryophyllia deshayesiana Michelin, 1850.

\section{DESCRIPTION}

Genre solitaire, assez grand, à squelette robuste et denté, de forme trochoïde sans stade discoïde à la base, à columelle spongieuse.

Acanthophyllia ampla (Reuss, 1871)

(Fig. 8A, B)

Lithophyllia ampla Reuss, 1871: 231, pl. 6, fig. 2. — Neugeboren 1876: 46.

Acanthophyllia ampla - Wells 1956: F417, fig. 319, 1, a-b.

MATÉRIEL. - Deux exemplaires de Lăpugiu, spécimen figuré: MNHN.F.A59929.

RÉPARTITION STRATIGRAPHIQUE ET GÉOGRAPHIQUE. - Miocène moyen: Hongrie (Reuss 1871), Roumanie (dont Lăpugiu qui est la localité type de l'espèce, ce travail).

\section{DESCRIPTION}

Les caractéristiques de $A$. ampla, hormis les caractères génériques, sont six cycles incomplets de septes (soit entre 96 et 192 septes, selon la taille de l'individu) et un diamètre calicinal pouvant atteindre $9 \mathrm{~cm}$, ovale, à côtes toutes identiques.

\section{Genre Syzygophyllia Reuss, 1860}

ESPÈCE TYPE. — Syzygophyllia brevis Reuss, 1860.

\section{DESCRIPTION}

Genre colonial, phacéloïde, à squelette fortement denté, à liaison lamellaire entre les centres calicinaux.

\section{Syzygophyllia (Syzygophyllia) crenaticosta (Reuss, 1869)} (Fig. 8C, D)

Rhabdophyllia crenaticosta Reuss, 1869: 237, pl. 18, figs 4-6.

Syzygophyllia (Syzygophyllia) crenaticosta - Cahuzac \& Chaix 1996: 117, 119 (avec erreur de date [1868 au lieu 1869] dans l'auteur du taxon).

MATÉRIEL. - Deux exemplaires de Lăpugiu, spécimen figuré: MNHN.F.A59930.

RÉPARTITION STRATIGRAPHIQUE ET GÉOGRAPHIQUe. — Oligocène inférieur: Italie (Reuss 1869).

Miocène inférieur: France (Cahuzac \& Chaix 1996)

Miocène moyen: Roumanie (Lăpugiu, ce travail).

\section{DESCRIPTION}

Cette espèce, en dehors des caractères génériques, se distingue par ses quatre cycles incomplets de septes. C'est la seule espèce du genre à avoir si peu de septes.
Syzygophyllia (Syzygophyllia) grandis Chevalier, 1962

(Fig. 8E, F)

Syzygophyllia grandis Chevalier, 1962a: 282, pl. 15, figs 13-15.

Syzygophyllia (Syzygophyllia) grandis-Cahuzac \& Chaix 1996: 112, 117, 119 (avec erreur de date [1961 au lieu 1962] dans l'auteur du taxon).

MATÉRIEl. - Cinq exemplaires de Lăpugiu, spécimen figuré: MNHN.F.A59931.

RÉPARTITION STRATIGRAPHIQUE ET GÉOGRAPHIQUE. - Oligocène supérieur: France (Cahuzac \& Chaix 1996).

Miocène inférieur: France (Cahuzac \& Chaix 1996).

Miocène moyen: Italie (Chevalier 1962a), Roumanie (Lăpugiu, ce travail).

\section{DESCRIPTION}

Espèce voisine de la précédente, elle ne s'en distingue que par ses cinq cycles incomplets de septes et son diamètre calicinal pouvant atteindre $30 \mathrm{~mm}$.

Famille FLABELLIDAE Bourne, 1905

Genre Flabellum Lesson, 1831

ESPÈCE TYPE. — Flabellum pavoninum Lesson, 1831.

\section{DESCRIPTION}

Genre solitaire, non récifal, de morphologie turbinée à trochoïde, à septes non dentés et columelle trabéculaire.

\section{Flabellum sp.}

(Fig. 8G)

COMMENTAire. - Seul un exemplaire (MNHN.F.A59932) de Flabellum a été récolté à Roșcani et il n'est pas identifiable spécifiquement.

Famille PORITIDAE Gray, 1842

Genre Porites Link, 1807

ESPÈCE TYPE. - Madrepora porites pars Pallas, 1766.

\section{DESCRIPTION}

Genre colonial, à squelette poreux, cérioïde, à petits calices, vivant en récifs. Columelle rudimentaire, trabéculaire.

\section{Porites arenosa (Esper, 1797)}

(Fig. 9A)

Madrepora arenosa Esper, 1797: 80, tableau LXV.

Porites arenacea - Lamarck 1816: 270 (2è éd. p. 455). — Deslongchamps et al. 1824: 651. — Ehrenberg 1834: 119. — Dana 1846: 567.

Porites arenaceus - de Blainville 1826: 50.

Porites cf. collegniana - Chevalier in Rangheard 1969: 287. 


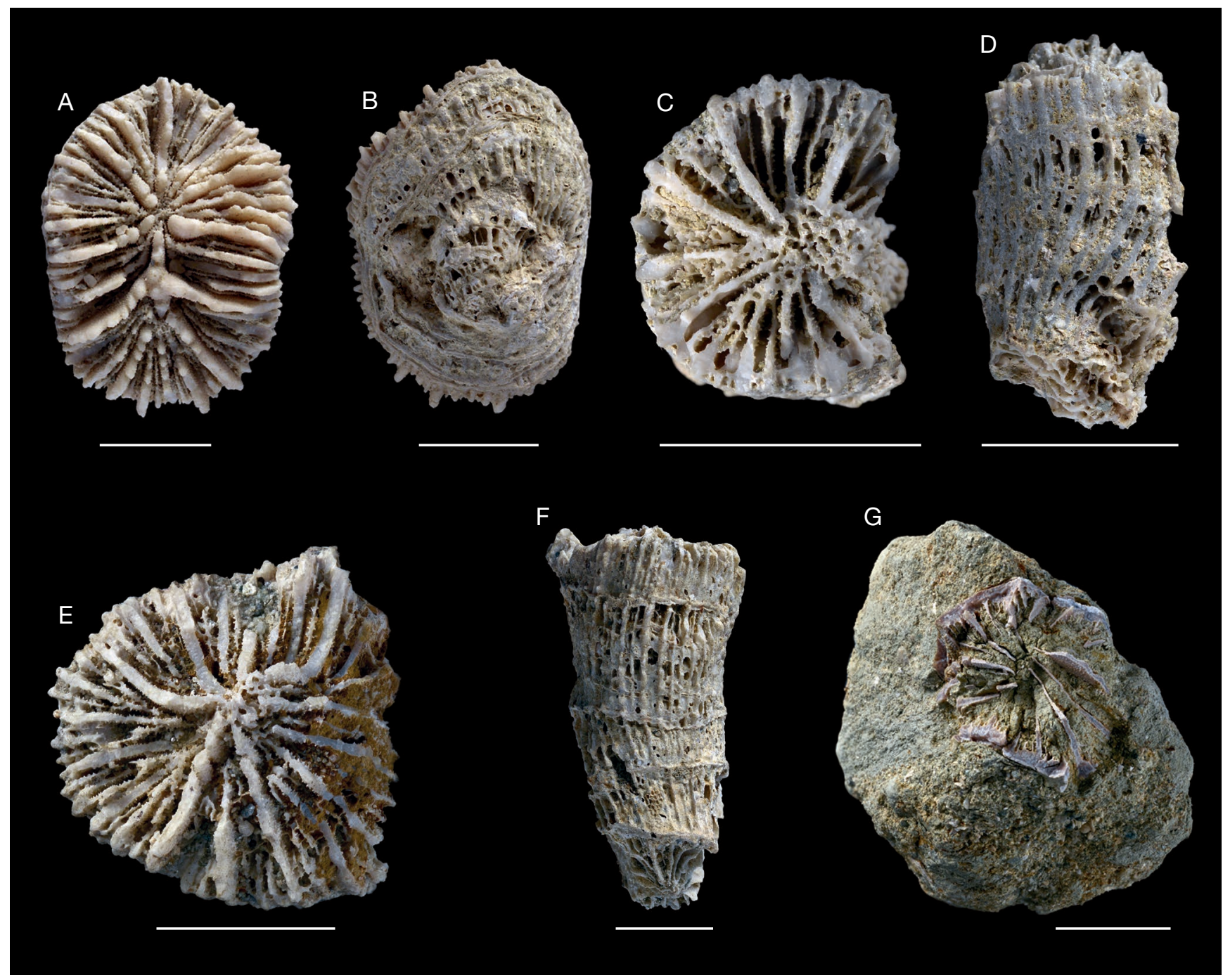

Fig. 8. - A, B, Acanthophyllia ampla (Reuss, 1871), MNHN.F.A59929; C, D, Syzygophyllia (Syzygophyllia) crenaticosta (Reuss, 1869), MNHN.F.A59930 ; E, F, Syzygophyllia grandis Chevalier, 1962, MNHN.F.A59931; G, Flabellum sp., MNHN.F.A59932. Échelles : $1 \mathrm{~cm}$.

Porites arenosa-Milne-Edwards \& Haime 1851:29; 1860: 180. Cahuzac \& Chaix 1996: 117, 119.

MATÉRIEL. - Deux exemplaires de Lăpugiu, spécimen figuré: MNHN.F.A59933.

RÉPARTITION STRATIGRAPHIQUE ET GÉOGRAPHIQUE. — Miocène inférieur: France (Cahuzac \& Chaix 1996).

Miocène moyen : les faunes des localités de cet âge dans lesquelles pourrait se rencontrer cette espèce (Italie par exemple) n'ont pas été révisées récemment, nos récoltes permettent de signaler l'espèce pour la première fois au Miocène moyen.

Miocène supérieur: Espagne, Grèce, Crète (Chaix \& Saint Martin 2008), Algérie (Chaix \& Saint Martin 2008), Baléares (Chaix \& Saint Martin 2008), Maroc (Chaix \& Saint Martin 2008), Italie, Sicile (Chaix \& Saint Martin 2008).

Pliocène: faunes non révisées.

Actuel: tout l'Indo-Pacifique (Milne-Edwards \& Haime 1860).

\section{DESCRIPTION}

Ce taxon a récemment été subdivisé en de multiples espèces (cf. Veron 2000a, b, c) dont l'identification repose sur des caractères extrêmement variables en fonction de l'environnement (morphologie de la colonie, "épaisseur» du squelette, pali plus ou moins nets, etc.). Pourtant, cette espèce, telle que décrite par Milne-Edwards \& Haime (1860) est très caractéristique et facile à identifier : 12 septes, une couronne de pali peu développés, une columelle nette faite d'une papille, un diamètre calicinal moyen mais peu variable de $1,5 \mathrm{~mm}$.

\section{Porites pusilla Felix, 1884}

(Fig. 9B)

Porites pusilla Felix, 1884: 445, pl. V, fig. 6. — Cahuzac \& Chaix 1996: 113, 119.

Non Porites leptoclada Reuss, 1871 - Rus \& Popa 2008: 330, pl. 3, fig. 9.

MATÉRIEL. - 103 exemplaires de Lăpugiu, spécimen figuré: MNHN.F.A59934. 
RÉPARTITION STRATIGRAPHIQUe ET GÉOGRAPHIQUe. - Oligocène supérieur: France (Cahuzac \& Chaix 1996).

Miocène inférieur: France (Cahuzac \& Chaix 1996).

Miocène moyen: Roumanie (Lăpugiu, ce travail).

Miocène supérieur: Égypte (Felix 1884).

\section{RAPPORTS ET DIFFÉRENCES}

Cette espèce se distingue des autres par ses 12 septes par calice et son diamètre calicinal de $1 \mathrm{~mm}$.

Porites maigensis gamachotensis Chevalier, 1962 (Fig. 9C)

Porites maigensis gamachotensis Chevalier, 1962a: 453, pl. XXI, fig. 21, pl. XXVI, fig. 2 et text-fig. 168). — Cahuzac \& Chaix 1996: 117.

MATÉRIEL. - Cinq exemplaires de Lăpugiu, spécimen figuré: MNHN.F.A59935

Répartition STRATigraphique et GÉographique. - Miocène inférieur: France (Chevalier 1962a; Cahuzac \& Chaix 1996).

Miocène moyen: Roumanie (Lăpugiu, ce travail).

RAPPORTS ET DIFFÉRENCES

Cette sous-espèce présente une morphologie lamellaire, un nombre de septes compris entre 12 et 15 , et un diamètre calicinal de $1,5 \mathrm{~mm}$.

\section{Porites collegniana lobatosepta Chevalier, 1962} (Fig. 9D)

Porites lobatosepta Chevalier, 1962a: 454, pl. XX, fig. 14 et pl. XXI, fig. 14.

Porites collegniana lobatosepta - Chaix \& Saint Martin 2008: 200, fig. 5C.

MATÉRIEl. - Deux exemplaires de Lăpugiu, spécimen figuré : MNHN.F.A59936.

Répartition stratigraphique eT GÉOgRaphique. - Miocène moyen: Roumanie (Lăpugiu, ce travail), cette sous-espèce étant signalée pour la première fois au Miocène moyen.

Miocène supérieur: Algérie et Maroc (Chaix \& Saint Martin 2008), Espagne (Chaix \& Saint Martin 2008), Italie (Chevalier 1962a; Chaix \& Saint Martin 2008), Grèce, Crète (Chaix \& Saint Martin 2008).

\section{DESCRIPTION}

Exactement la même que pour Porites collegniana collegniana, seule la morphologie de la colonie differe: rameuse dichotomique au lieu de massive; ce n'est pas en principe un critère de distinction spécifique car trop variable en fonction de l'environnement local, aussi ne l'avons-nous pas maintenue au rang d'espèce telle que Chevalier l'avait établie. Cependant, nous en faisons un critère de distinction subspécifique car il y a trop peu d'intermédiaires entre les deux formes pour tout intégrer dans une seule sous-espèce. Porites collegniana lobatosepta représente sans doute une forme d'adaptation, extrêmement constante, de $P$. collegniana aux conditions de la Méditerranée occidentale au Miocène supérieur.
Porites mancietensis Chevalier, 1962

(Fig. 9E)

Porites mancietensis Chevalier, 1962a: 454, pl. XXI, fig.13, pl. XXVI, fig. 6 et tf. 169, 171a. — Cahuzac \& Chaix 1996: 121. — Chaix \& Cahuzac 2005: tableau 5, pl. I, fig. 6.

MATÉRIEL. - Un exemplaire de Roșcani, spécimen figuré: MNHN.F.A59937.

Répartition STRATigraphiQue et GÉOgraphiQue. - Miocène moyen: France (Chevalier 1962a), Roumanie (Roșcani, ce travail).

\section{RAPPORTS ET DIFFÉRENCES}

Cette espèce se distingue nettement des autres par ses minuscules calices $(1 \mathrm{~mm})$ et ses trois cycles incomplets de septes (entre 12 et 16), P. pusilla ne développant jamais plus de 12 septes.

Genre Alveopora de Blainville, 1830

EsPÈCE TYPE. - Madrepora daedalea Forskael, 1775.

\section{DESCRIPTION}

Genre voisin de Porites, mais à squelette extrêmement poreux, très peu dense, à septes réduits à des épines séparées les-unes des autres, disposées de manière peu ou pas rayonnante.

Alveopora meridionalis Chevalier, 1962

(Fig. 9F)

Alveopora meridionalis Chevalier, 1962a: 440, pl. XX, fig. 1, 7, 9 et tf. 159. — Cahuzac \& Chaix 1996: 113.

MATÉRIEL. - Un exemplaire de Lăpugiu, spécimen figuré: MNHN.F.A59938.

RÉPARTITION STRATIGRAPHIQUE ET GÉOGRAPHIQUE. - Oligocène supérieur: France (Cahuzac \& Chaix 1996).

Miocène moyen: Roumanie (Lăpugiu, ce travail).

\section{DESCRIPTION}

Cette espèce, hormis les caractères génériques, se reconnaît à son nombre de rangées d'épines septales (entre 12 et 22) et son diamètre calicinal (4 mm maxi).

\section{Famille DeNDrophylliIDAE Gray, 1847}

Genre Balanophyllia Wood, 1844

ESPÈCE TYPE. - Balanophyllia calyculus Wood, 1844.

\section{DESCRIPTION}

Genre solitaire, à squelette poreux, fixé (par opposition avec son sous-genre Eupsammia), à éléments radiaires disposés suivant le plan de Pourtalès, et à columelle spongieuse. 


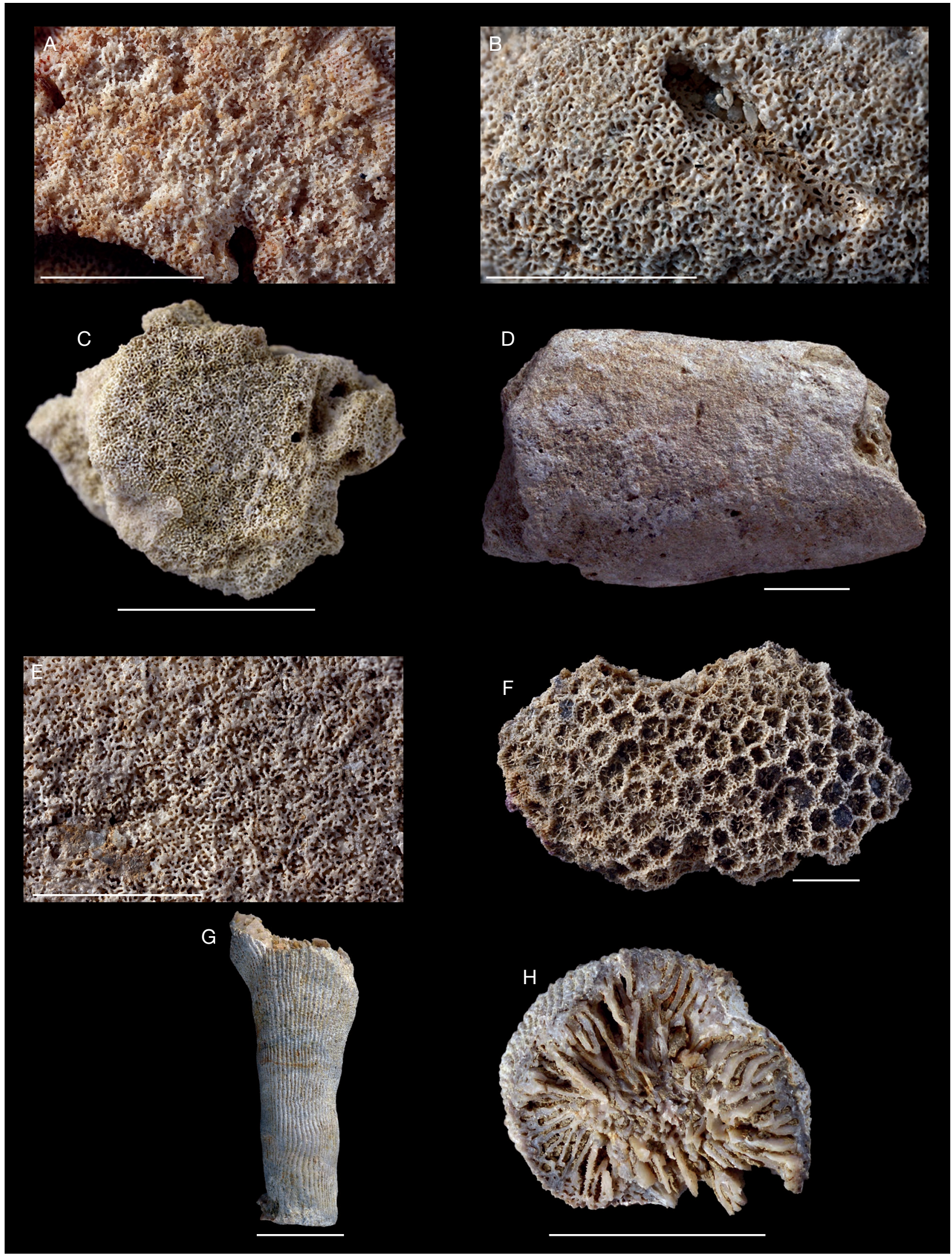

FIG. 9. - A, Porites arenosa (Esper, 1797), MNHN.F.A59933; B, Porites pusilla Felix, 1884, MNHN.F.A59934 ; C, Porites maigensis gamachotensis Chevalier, 1962, MNHN.F.A59935 ; D, Porites collegniana lobatosepta Chevalier, 1962, MNHN.F.A59936 ; E, Porites mancietensis Chevalier, 1962 , MNHN.F.A59937 ; F, Alveopora meridionalis Chevalier, 1962, MNHN.F.A59938; G, H, Balanophyllia varians Reuss, 1854, MNHN.F.A59939. Échelles : A, B, E, 0,5 cm; C, D, F-H, 1 cm. 
Balanophyllia varians Reuss, 1854

(Fig. 9G, H)

Balanophyllia varians Reuss, 1854: 747, 751. - Chevalier 1962a: 461, text-fig. 175c et 176c (avec synonymie). - Cahuzac \& Chaix 1996: $113,121$.

Cyathina multicostata Reuss, 1847: 15.

MATÉRIEL. - Un exemplaire de Lăpugiu (MNHN.F.A59939).

RÉPARTITION STRATIGRAPHIQUE ET GÉOGRAPHIQUE. - Oligocène supérieur: France (Cahuzac \& Chaix 1996).

Miocène moyen: France (Chevalier 1962a) Italie (Chevalier 1962a), Pologne (Reuss 1854) République tchèque (Reuss 1847), BosnieHerzégovine (Reuss 1854), Roumanie (Lăpugiu, ce travail).

Pliocène: France (Hallegouet et al. 1995; Chaix 1989; Chevalier et al. 1989; Lauriat-Rage et al. 1989), Grèce (Rhodes, récoltes Chaix 1997).

\section{DESCRIPTION}

Le plan de Pourtalès est très irrégulier dans cette espèce (d'où son nom). Elle possède cinq cycles incomplets d'éléments radiaires (soit 96 septes), des côtes toutes identiques, et une columelle peu à moyennement développée. Le rapport $\mathrm{d} / \mathrm{D}$ oscille entre 0,81 et 1 . La priorité ne peut s'appliquer pour Cyathina multicostata, cette espèce n'ayant été que citée, sans diagnose ni figuration; c'est Reuss en 1854 qui signale la synonymie des 2 espèces, en donnant une bonne description de Balanophyllia varians.

\section{ESPÈCES CITÉES DE LĂPUGIU ABSENTES DE NOTRE MATÉRIEL}

Plusieurs espèces de Faviidae ont été antérieurement décrites et citées (Tableau 1): Faviamagnifica Reuss, 1871 (Reuss \& Neugeboren ?1876), Favia somaliensis Gregory, 1900 (Rus \& Popa (2008) sous le nom Favia gotschevi Kojumdgieva, 1960), Favites conferta (Sismonda, 1871) (Rus \& Popa (2008) sous le nom Montastrea sp.), Heliastraea (Aquitanastraea) ramea Duncan, 1863 (Reuss 1871 et Neugeboren 1876), Heliastraea (Heliastraea) oligophylla Reuss, 1871 (Reuss 1871 et Neugeboren 1876) et Cladocora prevostana Milne-Edwards \& Haime, 1849 (Reuss 1871 et Neugeboren 1876). Deux espèces de Mussidae ont aussi été citées: Acanthophyllia spinosa (Gerth, 1921) (in Rus \& Popa 2008) sous le nom de Syzygophyllia brevis Reuss, 1860) et Syzygophyllia brevis Reuss, 1860 (Reuss 1871 ; Neugeboren 1876).

Reuss (1871) et Neugeboren (1876) ont décrit des coraux solitaires du genre Stephanophyllia: Stephanophyllia imperialis Michelin, 1841 et S. elegans (Bronn, 1838). Des espèces de Caryophylliidae et de Flabellidae, formes solitaires, ont aussi été citées (Reuss 1871; Neugeboren 1876; Halaváts 1876; Rus \& Popa 2008; cf. Tableau 1). Enfin, notre matériel ne comporte pas les espèces de Dendrophylliidae précédemment signalées (cf. Tableau 1). Les Dendrophylliidae affectionnant généralement des milieux profonds, leur rareté dans notre matériel provient probablement du fait que nos récoltes ont été menées dans les faciès littoraux.

\section{DISCUSSION}

\section{IMPLICATIONS PALÉOENVIRONNEMENTALES}

La présence dans leurs cellules gastrodermiques de zooxanthelles, algues unicellulaires symbiotiques, caractérise certains genres de coraux (zoxanthellés ou z-coraux). De faibles profondeurs favorisant la photosynthèse des zooxanthelles et des eaux chaudes constituent des facteurs donnant en général à ces coraux la capacité à construire des récifs. Wells (1933) a ainsi introduit la notion de coraux hermatypiques (ou récifaux) pour caractériser cette aptitude. De la même façon, les genres de coraux dépourvus de zooxanthelles étaient alors désignés comme ahermatypiques (ou non récifaux). L'équivalence entre la présence des zooxanthelles et le caractère hermatypique a été largement adoptée dans la littérature car elle semble vérifiée pour la grande majorité des coraux (par exemple pour des références assez récentes: Kucera 2009; Hopley 2011; Zeigler 2014; Cohen et al. 2016). Cependant, il a été noté quelques exceptions: certains genres de coraux à zooxanthelles ne construisent pas de récifs et des genres de coraux sans zooxanthelles peuvent être à l'origine de constructions importantes, surtout en eaux profondes (Schuhmacher \& Zibrowius 1985; Zibrowius 1988; Stanley \& Cairns 1988). Pour cette raison une classification tripartite peu usitée a été proposée permettant de prendre également en compte le problème des récifs anciens: la présence ou non de zooxanthelle (z-coraux/ non z-coraux), la capacité ou non à construire des récifs dans des eaux peu profondes (hermatypique/ahermatypique), la capacité ou non à former des structures élevées durables en eaux profondes ou peu profondes (constructeurs/non constructeurs). Quoi qu'il en soit, le caractère zooxanthellé des coraux constitue un élément important pour la reconstitution des environnements en matière de thermophilie, de bathymétrie, de potentiel récifal. En ce qui concerne le Cénozoïque avec des faunes coralliennes très proches de l'Actuel, on peut estimer comme z-coraux des genres possédant des zooxanthelles encore représentés aujourd'hui ou lorsqu'il s'agit de genres ayant la morphologie et les caractères du squelette des coraux connus comme des participants à la construction de récifs actuels (Bosellini \& Perrin 2008).

L'abondance des coraux hermatypiques dans la succession du Langhien de Lăpugiu traduit naturellement la mise en place de conditions climatiques tropicales propices, ce qui en fait un indicateur invoqué pour estimer la température des eaux (Chira et al. 2000). Ce contexte paléoclimatique chaud a été également mis en avant à partir de données diverses. La malacofaune, par exemple, présente une grande richesse en espèces thermophiles, particulièrement parmi les Conidae et les Cypraeidae (Chira et al. 2000; Chira \& Voia 2001). Un assez grand nombre de genres d'autres familles de gastéropodes ont été récoltés avec la faune de coraux et complètent encore le cortège de formes thermophiles. Il s'agit de Persististrombus Kronenberg \& Lee, 2007 (Strombidae), Tibia Gabb, 1868 (Rostellariidae), Xenophora Troschel, 1852 (Xenophoridae), Cassis Scopoli, 1777 (Cassidae), Aspa H. Adams \& A. Adams, 1853 (Bursidae), Ficus Röding, 1798 (Ficidae), Chicoreus (Triplex) Perry, 1810, Pterynotus Swainson, 1833, Homalocantha 
Mörch, 1852 et Vitularia Swainson, 1840 (les quatre genres de Muricidae), Melongena Schumacher, 1817 (Melongenidae), Tarantinaea Monterosato, 1917 (Fasciolariidae), Lyria Gray, 1847 et Athleta Conrad, 1853 (Volutidae), Oniscidia Mörch, 1852 (Harpidae), Anazola Gray, 1853 et Amalda H. Adams \& A. Adams, 1853 (Olividae), ainsi que plusieurs genres de Cancellaridae et de Terebridae. Parmi les bivalves, Demarcq (1987) mentionne une grande diversité de Pectinidae indiquant la présence d'eaux claires et chaudes. Selon Chira et al. (2000), l'assemblage malacologique indiquerait une température moyenne des eaux supérieure à $21^{\circ}$. Harzhauser et al. (2003) ont montré en outre que le Badénien inférieur a été marqué en Paratéthys centrale par un développement d'espèces de mollusques thermophiles. Des indices d'eaux chaudes sont aussi donnés par le nannoplancton calcaire et la présence de spicules d'ascidies propres aux environnements récifaux (Chira \& Marunțeanu 1999, 2000). Les données palynologiques font état d'une dominance d'éléments thermophiles dans le spectre pollinique (Petrescu et al. 1990; Țabără \& Chirilă 2012). Toutes ces indications allant dans le sens d'un épisode à caractère chaud, voire subtropical, sont sans doute à relier à l'optimum climatique global du Miocène Moyen (Zachos et al. 2001) mis en évidence en Paratéthys (Böhme 2003; Báldi 2006; Harzhauser \& Piller 2007). Du point de vue paléoocéanographique, la phase chaude du début du Badénien coïncide avec l'invasion de faunes indo-méditerranéennes (Moisescu \& Popescu 1980; Chira et al. 2000; Harzhauser \& Piller 2007; Harzhauser et al. 2007; Landau et al. 2009). Cette période serait marquée par la réouverture des communications avec le domaine océanique Indo-Pacifique (Rögl 1998, 1999) provoquant selon Karami et al. (2011) un réchauffement des eaux d'environ $3-4^{\circ}$, ce qui aurait été bien sûr favorable au développement d'une faune à caractère subtropical comme celle des coraux hermatypiques.

Labondance et la diversité des coraux hermatypiques dans les niveaux de Lăpugiu indiquent également classiquement des eaux très peu profondes. Les informations fournies par plusieurs groupes d'organismes représentés dans l'ensemble de la faune, comme les mollusques corroborent cette donnée essentielle pour la reconstitution du paléoenvironnement (Chira \& Voia 2001; Tămaş et al. 2013). Ainsi, la présence simultanée en abondance des gastéropodes Conidae, Cypraeidae, Terebridae, Harpidae et Cerithiidae (genre Thericium Monterosato, 1890) signent des environnements à substrats meubles mêlés à quelques substrats durs (ex: des coraux transportés) qui sont assez semblables aux fonds sableux coralliens actuels. La plupart de ces formes de gastéropodes sont sténohalines. Ce cortège de formes affectionnant de faibles profondeurs se rencontrent aussi avec des Bittium Gray, 1847, des Rissoidae, des Rissoinidae et des Jujubinus Monterosato, 1884 qui vivent dans des milieux riches en végétaux (herbiers) et indiquent un milieu de vie dans la zone euphotique. Toutes ces formes sont aussi mélangées à des Terebralia Swainson, 1840, des Melanopsis Férussac, 1807 et des Granulolabium Cossmann, 1889 qui suggèrent des apports ponctuels d'environnements fluviatiles et lagunaires.
Il est à remarquer que la croissance d'une grande variété de coraux ne se traduit en rien par une évolution vers un milieu de type récifal à proprement parler. Pratiquement aucun échantillon ne montre l'évidence de rapports de proximité (colonies poussant sur ou aux dépens d'autres colonies) ou de compétition entre les espèces coralliennes. De véritables constructions, de taille relativement modeste, sont cependant connues dans d'autres bassins néogènes proches, sous des faciès de type Leithakalk riches en algues calcaires et en constructions où la biodiversité corallienne est alors beaucoup plus faible (Saint Martin et al. 2007). Riegl \& Piller (2000a) ont proposé une classification de trois faciès à coraux du Badénien: 1) récifs coralliens-biohermes (coral reef-bioherm); 2) tapis coralliens-biostromes (coral carpet-biostrome); et 3) communautés coralliennes non constructrices (non-framework coral communities). Malgré le fait que les communautés coralliennes ne sont jamais retrouvées en place dans le sédiment, on peut considérer que les coraux du site de Lăpugiu correspondent assez bien à la dernière catégorie, caractérisée uniquement par une juxtaposition latérale des colonies latérale et par l'absence de succession verticale. Une telle caractéristique est aussi remarquée dans les gisements à coraux du Miocène d'Aquitaine (Cahuzac \& Chaix 1993, 1996).

Les données de terrain et la composition de la faune préservée à Lăpugiu suggèrent donc un environnement instable de fonds coralliens côtiers, riches en mollusques, où croissent des espèces coralliennes variées aux colonies cependant peu volumineuses, d'un ordre inférieur au mètre. Cette instabilité ne permet pas l'agrégation dans le temps des colonies et donc la formation de récifs. La paléogéographie littorale devait se présenter sous forme de baies peu profondes échancrées par des cours d'eau. Vers le centre du bassin la profondeur pouvait cependant être plus importante comme le suggèrent certaines faunes recensées, la profondeur pouvant varier ainsi entre quelques mètres et environ 90 mètres (Petrescu et al. 1990 ; Chira \& Voia 2001). La fréquence des épisodes terrigènes indique que ces environnements côtiers étaient soumis de manière récurrente à des décharges soudaines amenant du matériel continental dans le milieu marin et remaniant les dépôts antérieurs. L’abondance des restes végétaux, plus ou moins lignifiés selon les cas, traduit aussi cette influence continentale. La présence d'un cône de pin préservé dans le sédiment a ainsi été signalée (Givulescu \& Codrea 1997), ce qui est confirmé par la récolte lors de nos investigations d'un autre cône de pin dans les mêmes niveaux fossilifères.

Le recensement synthétique des coraux de Lăpugiu à partir de la littérature et les nouvelles récoltes permettent d'établir une liste, sans doute non exhaustive, de 65 espèces. Par rapport aux travaux antérieurs on note un enrichissement de 22 espèces nouvellement identifiées. Cette importante biodiversité corallienne, exceptionnelle pour un seul site, peut se décliner en 21 espèces de coraux solitaires et coloniales ahermatypiques, et 42 espèces de z-coraux. Aucun autre bassin miocène de Roumanie ne présente une telle diversité. Des coraux scléractiniaires ont cependant été signalés (Fig. 1A) dans le bassin de Bahna (Macovei 1909; Tița 2007), le bassin de Zarand (Nicorici \& Sagatovici 1973), le bassin de 
Beiuș (Păuca 1936), le bassin de Caransebeș-Mehadia (Pop 1960 ; Iliescu et al. 1968; Tița 1999, 2000) et le bassin de Transylvanie occidentale (Koch 1900; Saint Martin et al. 2007). La liste des scléractiniaires hermatypiques cités dans ces travaux fait apparaître surtout la dominance des genres Tarbellastraea et Heliastraea (Tableau 1). Dans certains cas ce sont surtout des coraux solitaires qui sont décrits (Tița 1999, 2000), surtout en milieux marneux et profond.

Si la biodiversité corallienne de Lăpugiu apparaît remarquable dans le contexte des bassins néogènes de Roumanie, il est intéressant d'élargir le champ paléogéographique à la Paratéthys centrale. Généralement reliée aux faciès de type Leithakalk, la faune corallienne a été recensée dans de nombreux pays avec une amplitude latitudinale qui va de la Bulgarie à la Pologne (Pisera 1996). C'est essentiellement dans les bassins néogènes autrichiens (bassin de Vienne et bassin styrien) que les travaux sur les dépôts badéniens ont été les plus nombreux, permettant d'établir des listes de biodiversités coralliennes (Reuss 1847; Reuss 1871; Schouppé 1949; Kühn 1963; Muckenhuber 1964; Dullo 1983; Friebe 1988, 1990, 1991, 1993; Riegl \& Piller 2000a, b; Piller \& Kleemann 1991; Schmid et al. 2001; Gross et al. 2007; Reuter \& Piller 2011; Reuter et al. 2012; Wiedl et al. 2012, 2013; Reuter \& Piller 2014). Quelques coraux sont signalés dans le Badénien de la République tchèque (Doláková et al. 2008). Géographiquement plus proches, plusieurs gisements du Badénien inférieur de Hongrie ont fourni une faune corallienne également intégrée dans des dépôts de type Leithakalk (Hegedüs 1970; Scholz 1970; Hegedüs \& Jankovich 1970; Oosterbaan 1990; Saint Martin et al. 2000). D'autres indications peuvent être tirées de travaux portant sur plusieurs secteurs de l'ensemble balkanique: Croatie (Sremac et al. 2015), Serbie (Jovanović 2011), Bulgarie (Kojumdgieva \& Strachimirov 1960). Enfin, les faunes coralliennes les plus nordiques ont été décrites en Pologne, avec cependant un nombre d'espèces hermatypiques limité (Roszkowska 1932; Roniewicz \& Stolarski 1991; Stolarski 1991; Górka 2002). La compilation de toutes ces données (Tabl. 1) fait apparaittre une biodiversité corallienne significativement plus importante à Lăpugiu, surtout si on considère qu'elle ne concerne qu'un seul site fossilifère, relativement limité dans l'espace. Cependant, Saint Martin et al. (2007) ont suggéré que la biodiversité des coraux hermatypiques dans les faciès de type Leithakalk en Roumanie pourrait se trouver minorée en raison des conditions taphonomiques et diagénétiques particulières aux environnements carbonatés (Dullo 1983), conditions plutôt défavorables à une bonne préservation des caractères diagnostiques des squelettes coralliens. Cette remarque vaut bien évidemment pour l'ensemble de la Paratéthys. On pourrait ainsi considérer que les gisements de type Lăpugiu reflètent une paléocommunauté de fonds coralliens (ou "non coral-framework community", Reuter et al. 2012) mieux préservée. D'autres explications sont également envisageables. Les conditions taphonomiques ont pu jouer un rôle dans la mesure où les colonies coralliennes ainsi d'ailleurs que les coquilles de mollusques ont été rapidement déplacées avant dégradation, redistribuées vers des fonds de baie plus protégés et moins oxygénés, et finalement recouverts de vases argileuses permettant une meilleure préservation de l'aragonite. Selon Kováč et al. (2007), la position latitudinale pourrait également constituer un facteur de diversification, les récifs coralliens étant situés plus au sud et les assemblages de coraux hermatypiques semblant plus pauvres au nord. De ce point de vue, la situation méridionale du bassin de Făget pourrait partiellement expliquer une certaine différenciation, mais seulement par rapport aux bassins situés sensiblement plus au nord (Hongrie, Autriche, Pologne...). Or, il est à remarquer que les faunes coralliennes recensées plus au Sud (Bulgarie par exemple) ne montrent pas plus de richesse. La paléogéographie particulière de la région de Lăpugiu au sein de paléoreliefs formant des terres espacées aurait pu constituer un contexte d'archipels, la mutiplication des îles favorisant le développement de faunes littorales. De plus, la position du bassin de Făget dans une zone de communication devait permettre des échanges et des circulations de larves propices à l'implantation corallienne sur les nombreux espaces côtiers. Enfin, la diversité corallienne de Lăpugiu est aussi à mettre en relation avec une grande variété de biotopes et de substrats dans des environnements, allant de l'infralittoral au circalittoral, dont les peuplements se trouvent bien souvent mêlés ou intercalés dans une série finalement peu épaisse. Cette dernière considération peut rejoindre les constatations de Zuschin et al. (2011) concernant les biais d'évaluation des paléobiodiversités selon la place dans un cycle de dépôt.

Selon Rus \& Popa (2008), la faune corallienne de Lăpugiu de Sus, remarquable par son abondance et sa diversité serait à mettre dans le contexte de mers du Miocène moyen caractérisées par l'abondance des coraux et l'acmé du développement récifal relié à l'Optimum Climatique du Miocène moyen ressenti mondialement (Kiessling et al. 1999). De fait, cette période correspond également à l'extension la plus septentrionale des édifices coralliens (Perrin 2002; Perrin \& Kiessling 2010; Vertino et al. 2014). La richesse taxinomique des coraux à zooxanthelles (z-genres) pourrait constituer un marqueur pour l'estimation des températures de surface des océans, et notamment pour le domaine paratéthysien où la compilation des données par Bosellini \& Perrin (2008) fait apparaître un maximum de $11 \mathrm{z}$-genres, mais sans prendre en compte les données du Badénien de Roumanie. En conséquence, la diversité des genres coralliens aussi bien dans le domaine méditerranéen qu'en Paratéthys semble plus basse qu'on pourrait s'y attendre en regard des paléotempératures supposées (Bosellini \& Perrin 2008, 2010). Le nombre de z-genres pour un seul site serait ainsi en moyenne inférieur à cinq, pouvant aller jusqu'à dix seulement occasionnellement (Perrin \& Bosellini 2012). Mais il est à remarquer que lorsque la diversité est proche de dix il ne s'agit pas en réalité d'un seul site mais d'une région ou d'un bassin. De ce fait, la richesse taxinomique en z-genres de Lăpugiu, qui jusqu’à présent n'a pas été bien prise en compte, pourrait être plus en rapport avec les indications climatiques attendues. Les 
informations tirées de ce seul gisement constituent de fait une référence nécessaire pour toute compréhension de la paléotempérature et la paléoocéanographie de cette période du Miocène moyen. Finalement, la biodiversité corallienne en z-genres et non z-genres apparaît comme la plus importante connue en Paratéthys.

\section{UN HOT SPOT DE LA BIODIVERSITÉ ?}

La biodiversité corallienne remarquable mise en évidence à Lăpugiu doit sans doute être aussi mise en rapport avec l'exceptionnelle biodiversité constatée pour d'autres groupes. Les fossiles de ce site figurent ainsi dans de très nombreuses collections muséales ou personnelles d'Europe. Ce sont surtout les mollusques (principalement les gastéropodes et les bivalves) qui ont attiré l'attention avec une liste impressionnante de plusieurs centaines d'espèces décrites ou citées par plusieurs auteurs depuis le 19ème siècle (Hörnes 1856; Neugeboren 1856a; Halaváts 1876; Neugeboren 1878; Hauer \& Stache 1863; Hoernes 1878; Hoernes \& Auinger 1879-1891; Boettger 1887; Boettger 1895; Koch 1898; Koch 1900; Boettger 1900; Boettger 1903-1904; Nițulescu 1930; Moisescu 1955), liste plus récemment encore mise à jour (Mienis 1975; Chira 1994; Pacaud 2003; Fehse \& Vicián 2008; Landau et al. 2009; Caze et al. 2010; Popa et al. 2014; Kovács \& Balázs 2015). Alors que Koch (1900) fait état de 833 espèces de mollusques, le nombre total à la suite des travaux plus récents pourrait atteindre le millier selon Chira \& Vioa (2001). D'autres groupes de macrofaune comme les mollusques polyplacophores (Dell'Angelo et al. 2007), les brachiopodes (Moisescu 1955; Brăbulescu \& Rado 1984; Dulai 2015) ou les annélides tubicoles (Meznerics 1944) ont fait également l'objet de recensement ou révisions. Les bryozoaires ont été étudiés par Ghiurcă (1961). Les microfaunes de foraminifères et d'ostracodes ont été depuis très longtemps largement détaillées (Neugeboren 1847, 1849-1850, 1851, 1852, 1856b, c, 1860, 1869; Şuraru \& Papp 1993; Chira \& Marunțeanu 1999, 2000; Chira \& Voia 2001; Boga 2013; Popescu \& Crihan 2005).

Une telle abondance et une telle biodiversité, y compris celles des coraux scléractiniaires, ne manquent donc pas d'attirer l'attention. Quelles que soient les divers facteurs qui concourent à cette richesse en fossiles, on peut légitimement estimer que la région de Lăpugiu correspond assez bien à une aire marine ayant montré une haute biodiversité taxinomique et fonctionnelle, critères pouvant déterminer le qualificatif de hotspot de la biodiversité fossile (Willis et al. 2006; Mihaljević et al. 2017), comme cela a été envisagé pour les gisements fossilifères éocènes du Bassin de Paris (Merle 2008; Renema 2011; Huyghe et al. 2012; Sanders et al. 2015). La notion de hotspot de la biodiversité des récifs coralliens, et conséquemment des coraux hermatypiques, a d'ailleurs été mise en avant pour montrer le déplacement des zones majeures de biodiversité marines en fonction de la tectonique des plaques (Leprieur et al. 2016). Le domaine méditerranéen représentait ainsi au Miocène moyen un espace de forte biodiversité. La biodiversité corallienne mise en évidence dans ce travail constituerait ainsi un élément à prendre largement en considération dans cette perspective.

\section{Remerciements}

Nous remercions sincèrement notre collègue Sorin Filipescu (Université Cluj-Napoca, Roumanie) pour les informations données sur le site de Lăpugiu et son aide bienveillante. Les photographies des spécimens figurés ont été réalisées par Lilian Cazes et Philippe Loubry (MNHN). Les revues de B. Lathuilière et de $\mathrm{G}$. Conesa ont grandement contribué à améliorer cet article.

\section{RÉFÉRENCES}

ACHIARDI A. D' 1868. - Studio comparativo fra I coralli dei terreni terziari del Piemonte e dell'Alpi venete. Pisa, Nistri, 74 p.

Alloiteau J. 1950. - Types et échantillons de polypiers de l'ancienne collection Defrance. Mémoires du Muséum national d'Histoire Naturelle de Paris série C, Sciences de la Terre 1 (2): 105-148.

Alloiteau J. 1957. - Contribution à la systématique des madréporaires fossiles. Éditions du CNRS, Paris, 2 volumes, $462 \mathrm{p}$.

BÁLDI K. 2006. - Paleoceanography and climate of the Badenian (Middle Miocene, 16.4-13.0 Ma) in the Central Paratethys based on foraminifera and stable isotope (d18O and d13C) evidence. International Journal of Earth Sciences 95: 119-142. https://doi.org/10.1007/s00531-005-0019-9

BRĂBULESCU A. \& RADO G. 1984. - Contributions à la connaissance des brachiopodes badéniens de Roumanie. 75 Years of the Laboratory of Paleontology, Special Volume: 173-183.

Blainville H. M. D. DE 1826. — Porite, in Cuvier F. (ed.), Dictionnaire des Sciences naturelles. Levrault, Strasbourg, Paris; Le Normant, Paris, XLIII: 49-52. https://biodiversitylibrary. org/page/25309114

Blainville H. M. D. DE 1830. — Zoophytes, in Cuvier F. (ed.), Dictionnaire des Sciences naturelles. Levrault, Strasbourg, Paris; Le Normant, Paris, LX: 1-546. https://biodiversitylibrary.org/ page/25318346

BlainVille H. M. D. DE 1834. - Manuel d'actinologie ou de zoophytologie, 2 vols. F. G. Levrault, Paris. 695 p. https://doi. org/10.5962/bhl.title. 8768

BoetTger O. 1887. - Drei neue Conus aus dem Miocaen von Lapugy und von Bordeaux. Jahrbucher der Deutschen Malakozoologischen Gesellschaft 14: 4-8.

BOETTGER O. 1895. - Zur Kenntnis der Fauna der mittelmiocänen Schichten von Kostej im Banat. Verhandlungen und Mittheilungen des Siebenbürg für Naturwissenschaften zu Hermannstadt 46: 49-66. https://biodiversitylibrary.org/page/33653148

Boettger O. 1900. - Zur Kenntnis der Fauna der mittelmiocänen Schichten von Kostej im Krassó-Szörényer Komitat. Verhandlungen und Mittheilungen des Siebenbürg für Naturwissenschaften zu Hermannstadt 51: 1-200. https://biodiversitylibrary.org/page/35260319

BoetTGer O. 1903-1904. - Zur Kenntnis der Fauna der mittelmiocänen Schichten von Kostej im Krassó-Szörényer Komitat (Gasteropoden und Anneliden). Verhandlungen und Mittheilungen des Siebenbürg für Naturwissenschaften zu Hermannstadt 54: 1-99 (https://biodiversitylibrary.org/page/34802831) ; 55: 101-244 (https://biodiversitylibrary.org/page/34803026).

BogA C. R. 2013. - The study of the foraminifera and ostracoda microfauna of the Badenian deposits from Coșului Valley, Lăpugiu de Sus, Hunedoara county. The 9th Romanian Symposium on Paleontology, Iași, Abstracts: 7.

Bosellini F. R. \& PerRin C. 2008. — Estimating Mediterranean Oligocene-Miocene sea surface temperatures: an approach based on coral taxonomic richness. Palaeogeography, Palaeoclimatology, Palaeoecology 258: 71-88. https://doi.org/10.1016/j. palaeo.2007.10.028 
Bosellini F. R. \& Perrin C. 2010. - Coral diversity and temperature: a palaeoclimatic perspective for the Oligo-Miocene of the Mediterranean region, in MutTi M., Piller W. E. \& Betzler C. (eds), Carbonate Systems During the Oligocene-Miocene Climatic Transition. IAS Special Publication 42: 227-242. https:// doi.org/10.1002/9781118398364.ch13

BöHme M. 2003. - The Miocene Climatic Optimum: evidence from ectothermic vertebrates of Central Europe. Palaeogeography, Palaeoclimatology, Palaeoecology 195: 389-401. https://doi. org/10.1016/S0031-0182(03)00367-5

BOURNE G. C. 1905. - Report on the solitary corals collected by Professor Herdman at Ceylon, in 1902, in HERDMAN W. A. (ed.), Report to the government of Ceylon on the pearl oyster fisheries in the Gulf of Manaar. Royal Society London, supplementary reports 29, part 4: 187-242. https://biodiversitylibrary.org/page/1936048

BronN H. G. 1838. — Lethaea geognostica. E. Schweizerbart's Verlagshandlung, band II: 769-1346.

Budd A. F., Bosellini F. R. \& Stemann T. A. 1996. - Systematics of the Oligocene to Miocene reef coral Tarbellastraea in the northern Mediterranean. Palaeontology 39 (3): 515-560. https:// biodiversitylibrary.org/page/49760986

CAHUZaC B. \& Chaix C. 1993. — Les faunes de coraux (Anthozoaires Scléractiniaires) de la façade atlantique française au Chattien et au Miocène. Proceedings of the 1st R.C.A.N.S. congress, Lisboa, October 1992. Ciências da Terral Earth Sciences Journal 12: 57-69.

CaHuzac B. \& Chaix C. 1996. - Structural and faunal evolution of Chattian-Miocene reefs and corals in western France and the northeastern Atlantic Ocean. SEPM, Society for Sedimentary Geology, Concepts in Sedimentology and Paleontology 5: 105-127. https://doi.org/10.2110/csp.96.01.0105

Catullo T. A. 1856. - Dei terreni di sedimento superiore delle Venezie e dei fossili Bryozoari, Antozoari e Spongiari. Angelo Sicca, Padova, $88 \mathrm{p}$.

Caze B., Saint Martin J.-P., Merle D. \& Saint Martin S. 2010. Intérêt des motifs colorés résiduels des coquilles de mollusques pour la valorisation des sites paléontologiques et des collections: l'exemple du Badénien de Roumanie, in SAINT MarTIN J.-P., SAINT Martin S., OAie G., Seghedi A. \& Grigorescu D. (ed.), $L e$ patrimoine paléontologique. GeoEcoMar, Bucarest: 27-38.

CHAIX C. 1989. - Les Scléractiniaires du Pliocène de Normandie. Bulletin du Muséum national d'Histoire naturelle, 4ème série, Section C, Sciences de la terre, paléontologie, géologie, minéralogie 11 (1): 3-13.

ChaIX C. \& CAHUZAC B. 2005. - Les faunes de Scléractiniaires dans les faluns du Miocène moyen d'Atlantique-Est (bassins de la Loire et d'Aquitaine) : paléobiogéographie et évolution climatique. Annales de Paléontologie 91 (1):33-72. https://doi.org/10.1016/j. annpal.2004.11.001

Chaix C. \& SAINT MARTIN J.-P. 2008. — Les faunes de scléractiniaires hermatypiques dans les plates-formes carbonatées méditerranéennes au Miocène supérieur, in SAINT MARTIN J.-P. (ed.), Biodiversity and biodiversity crises in North African Neogene. Geodiversitas 30 (1): 181-209.

Chaix C., Moissette P. \& Saint Martin J.-P. 1986. - Réflexions sur les biocénoses/taphocénoses en milieu récifal (Messinien d'Algérie). Bulletin du Muséum national d'Histoire naturelle, 4ème série, Section C, Sciences de la terre, paléontologie, géologie, minéralogie 8 (2): 219-230.

Chaix C., Galloni F., Ferrandini M. \& Cornée J. J. 2015. Les coraux scléractiniaires du Burdigalien supérieur du Bassin de Bonifacio (Corse-du-Sud). Geodiversitas 37 (3): 367-381. https:// doi.org/10.5252/g2015n3a5 https://doi.org/10.5252/g2015n3a5

CHeVAlier J.-P. 1954. - Contribution à la révision des polypiers du genre Heliastraea. Annales Hébert et Haug VIII: 106-190.

Chevalier J.-P. 1955. — Polypiers, in BrunN J. H., Chevalier J.-P. \& MARIE P. (ed.), Quelques formes nouvelles de polypiers et de foraminiferes de l'Oligocène et du Miocène du NW de la Grèce. Bulletin de la Société géologique de France, sér. 6, 5 (1-3): 195-202. https://doi.org/10.2113/gssgfbull.S6-V.1-3.193
Chevalier J.-P. 1962a. — Recherche sur les madréporaires et les formations récifales miocènes de la Méditerranée occidentale Atlas paléontologique. Thèse d'état (Paris). Mémoires de la Société géologique de France, série A, 93, 562 p.

CHEVALIER J.-P. 1962b. — Les Madréporaires miocènes du Maroc. Notes et Mémoires du Service géologique du Maroc 173: 5-74.

Chevalier J.-P. 1966. — Contribution à l'étude des Madréporaires des côtes occidentales de l'Afrique tropicale. Bulletin de l'Institut français d'Afrique noire, sér. A, 3-4: 912-975 et 1356-1405.

Chevalier J.-P. 1970. — Les Madréporaires du Néogène et du Quaternaire de l'Angola. Annales du Musée royal d'Afrique centrale, Sciences Géologiques 68: 11-32.

Chevalier J.-P. \& HÉBRARD L. 1972. — Découverte de Madréporaires dans le Pléistocène supérieur de Mauritanie. Congrès Panafricain de Préhistoire, Actes, Gème sér., Dakar 1967. Chambéry, Impr. Réunies: 453-456.

Chevalier J.-P. \& Nascimento A. 1975. - Notes sur la géologie et la paléontologie du Miocène de Lisbonne. XVI - Contrinution à la connaissance des madréporaires et des faciès récifaux du Miocène inférieur. Boletim de la Sociedade Geologica de Portugal 19 (3): 247-281.

Chevalier M., Borne V., Brébion P., Buge E., Chaix C., Courbouleix S., Delanoe Y., Esteoule-Choux J., Farjanel G., Lauriat-Rage A., Margerel J.-P. , Pouit D., Roman J., Tauth N. \& ViaUd J. M. 1989. — Le complexe cénozoïque de Corcouésur-Logne (Loire-Atlantique). Études sédimentologiques, paléontologiques et premières reconnaissances géophysiques. Géologie de la France 1-2: 153-170.

CHIRA C. 1994. - Catalogue of the Bivalvia collection of Lăpugiu de Sus preserved at the Paleontology-Stratigraphy Museum of the University of Cluj, in NiCORICI E. (ed.), The Miocene from the Transylvanian Basin-Romania. Editura Carpatica, Cluj-Napoca: 75-80.

CHIRA C., FILIPESCU S. \& CODREA V. 2000. — Palaeoclimatic evolution in the Miocene from the Transylvanian Depression reflected in the fossil record, in HART M. B. (ed.), Climates: past and present. Geological Society, London, Special Publications 181: 55-64. https://doi.org/10.1144/GSL.SP.2000.181.01.06

Chira C. \& Marunteanu M. 1999. — Middle Miocene (Lower Badenian) calcareous nannofossils from the Mures passageway and Făget basin, România. Acta Palaeontologica Romaniae 2: 73-82.

CHIRA C. \& MARUNȚEANU M. 2000. - Calcareous nannofossils and dinoflagellates from the Middle Miocene of the Transylvanian Basin, Romania. INA8 8th International Nannoplankton Association Conference Abstracts, 3 p.

Chira C. \& VoIA I. 2001. - Middle Miocene (Badenian) Conidae from Lăpugiu de Sus, Romania: Systematical and palaeoecological data. Studia Universitatis Babeş-Bolyai. Geologia 46 (2): 151-160. https://doi.org/10.5038/1937-8602.46.2.13

COHEN I., DubinSKY Z. \& EREZ J. 2016. — Light enhanced calcification in hermatypic corals: new insights from light spectral responses. Frontiers in Marine Science 2: 1-12. https://doi. org/10.3389/fmars.2015.00122

DANA J. D. 1846. - Structure and Classification of Zoophytes. Geological United States Exploring Expedition, Londres, 1838-1842, 7 (Zoophytes), 740 p. https://doi.org/10.5962/bhl.title.4789

Defrance M. 1826. - Polypier, in Cuvier F. (ed.), Dictionnaire des Sciences naturelles, Levrault, Strasbourg, Paris; Le Normant, Paris, XLII: 372-398. https://biodiversitylibrary.org/page/25308359

Dell'Angelo B., Grigis M. \& Bonfitto A. 2007. - Notes on fossil chitons. 2. Polyplacophora from the Middle Miocene of Lăpugiu (Romania). Bolletino Malacologico 43 (1-8): 39-50.

DemarcQ G. 1987. - Paleothermic evolution during the Neogene in Mediterranea through the marine megafauna. Magyar Állami Földtani Intézet évkönyve 70: 371-375.

DeslongChamps E., Lamouroux J. V. F. \& De SAint-Vincent B. 1824. - Histoire naturelle des Zoophytes ou animaux rayonnés. Encyclopédie méthodique, II-VIII, 819 p. https://doi.org/10.5962/ bhl.title.64045 
Doláková N., Brzobohatý R., Hladilová Š. \& Nehyba S. 2008. - The red-algal facies of the Lower Badenian limestones of the Carpathian Foredeep in Moravia (Czech Republic). Geologica Carpathica 59 (2): 133-146.

Dulai A. 2015. - Central Paratethyan Middle Miocene brachiopods from Poland, Hungary and Romania in the Naturalis Biodiversity Center (Leiden, the Netherlands). Scripta Geologica 149: 185-211.

Dullo W. C. 1983. - Diagenesis of fossils of the Miocene Leitha Limestone of the Paratethys, Austria: an example for faunal modifications due to changing diagenetic environments. Facies 8: 1-112. https://doi.org/10.1007/BF02536740

DUNCAN P. M. 1863. - On the fossil corals of the West Indian islands - Part I. Quarterly Journal of the Geological Society of London 19 (1-2): 406-458. https://biodiversitylibrary.org/page/35328377

Ellis J. \& SOlander D. 1786. - Natural History of Many Curious and Uncommon Zoophytes. Benjamin White \& Son, London, 206 p. https://doi.org/10.5962/bhl.title.64985

Ehrenberg C. G. 1834. — Beitrag zur Kenntniss der Corallenthiere des rothen Meeres. Abhandlungen der königlich-preussischen Akademie der Wissenschaften (Physische Classe): 225-380.

EsPer E. J. C. 1797. - Die Pflanzenthiere in Abbildungen nach der Natur mit Farben erleuchtet nebst Beschreibungen. Nürnberg, 230 p. https://doi.org/10.5962/bhl.title. 118730

FeHSE D. \& VICIÁN Z. 2008. - On the identity of Projenneria neumayri (Hilber, 1879) with the description of a new species of the genus Projenneria Dolin, 1997 from the Badenian of the Central Paratethys. Földtani Közlöny 138 (4): 357-362.

FELIX J. P. 1884. - Korallen aus ägyptischen Tertiärbildungen. Zeitschrift der Deutschen Geologischen Gesellshaft 36: 415-453.

FILIPESCU S. 2011. - Cenozoic lithostratigraphic units in Transylvania, in BUCUR I \& SĂSĂRAN E (eds), Calcareous Algae from Romanian Carpathians. Field trip Guidebook. Presa Universitară Clujeană: 37-48.

FORSKAEL P. 1775. - Descriptiones animalium, avium, amphibiorum, piscium, insectorum, vermium/quae in itinere orientali observavit. Hauniae, 163 p. https://doi.org/10.5962/bhl.title.2154

FrIEBE J. G. 1988. — Paläogeographische Überlegungen zu den Leithakalkarealen (Badenien) der Mittelsteirischen Schwelle (Steiermark). Geologische-Paläontontologische Mitteilungen Innsbruck 15: 41-57.

FrIEBE J. G. 1990. — Lithostratigraphische Neugliederung und Sedimentologie der Ablagerungen des Badenium (Miozän) um die Mittelsteirische Schwelle (Steirisches Becken, Österreich). Jahrbuch der Geologische Bundesanstalt 133 (2): 223-257.

FrIEBE J. G. 1991. - Middle Miocene reefs and related facies in Eastern Austria. II. Styrian basin. VI. International Symposium on Fossil Cnidaria including Archaeocyatha and Porifera, Münster 1991, Excursion B4 Guidebook: 29-47.

FRIEBE J. G. 1993. - Sequence stratigraphy in a mixed carbonate-siliciclastic depositional system (Middle Miocene; Styrian Basin, Austria). Geologische Rundschau 82: 281-294. https://doi. org/10.1007/BF00191834

Frost S. H. \& LangenHeim R. L. 1974. - Cenozoic Reef Biofacies. Tertiary Larger Foraminifera and Scleractinian Corals from Chiapas, Mexico. Northern Illinois University Press, De Kalb, Illinois, XI, 388 p.

Gerth H. 1921. - Anthozoa, in Martin K. (ed.), Die Fossilen von Java. Sammlungen des Geologischen Reichsmuseum in Leiden, n.s., vol. 1, 2 Abt., heft 3: 387-445.

GHIURCĂ V. 1961. - Contributii la cunoasterea faunei de bryozoare din Transilvania (II). Revizuirea taxonomica a bryozoarelor de la Lăpugiu si Buituri publicate de A. Koch. Studia Universitatis Babes-Bolyai. Series Geologia-Geographia 1: 137-140.

Givulescu R. \& Codrea V. 1997. - Pinus badenica n. sp. - A new Lower Badenian pine from Lăpugiu de Sus. Romanian Journal of Paleontology 77: 39-42.

GoCEV P. 1935. - Beitrag zur Kenntniss der Korallen des Tortons von Pleven (Nord Bulgarien). Zeitschrift der Bulgarischen Geologischen Gesellschaft, Jahrg. 7, Heft 1: 1-7.
Goldfuss G. A. 1826-1833. - Petrefacta germaniae. Aruz \& Cie, Dusseldorf, 252 p. https://doi.org/10.5962/bhl.title.115962

GÓRKA M. 2002. - The Lower Badenian (Middle Miocene) coral patch reef at Grobie (southern slopes of the Holy Cross Mountains, Central Poland), its origin, development and demise. Acta Geologica Polonica 52 (4): 521-533.

GraY J. E. 1842. - Synopsis of the Contents of the British Museum. 44th ed. British Museum, London IV, 308 p.

GRAY J. E. 1847. - An outline of an arrangement of stony corals. The Annals and Magazine of Natural History 19: 120-128. https:// doi.org/10.1080/037454809496460

GREGORY J. W. 1900. — Geology and fossil corals and echinoids of Somaliland. Quarterly Journal of the Geological Society of London 56: 26-45. https://doi.org/10.1144/GSL.JGS.1900.056.01-04.06

Gregory J. W. 1906. — Fossil corals from eastern Egypt, Abu Roasch and Sinai. Geological Magazine 5 (3): 50-58, 110-118. https://doi.org/10.1017/S0016756800122885

Gross M., Fritz I., Piller W. E. , Soliman A., Harzhauser M., Hubmann B., Moser B., Scholger R., SutTner T. C. , Bojar H.-P. 2007. - The Neogene of the Styrian Basin - Guide to excursions. Joannea Geologie und Paläontologie 9: 117-193.

HAIME J. 1852. - Catalogue raisonné des fossils nummulitiques du comté de Nice, in Bellardi L. (ed.), Mémoires de la Société géologique de France, série 2, 4: 205-300. https://biodiversitylibrary.org/page/42342219

HalaVÁts G. 1876. — Die mediterrane Fauna von Felsö-Lapugy. Földtani Közlöny 6: 229-240.

Hallegouet B., Margerel J.-P., Lauriat-Rage A., Carbonel P., CarRIOL R. P. \& CHAIX C. 1995. — Le Pliocène de Brest-Landerneau (Finistère, France). Interim Colloquium RCANS: Atlantic neogene climates and paleoenvironnements (24-27 Mai 1995, Tours): 13.

Harzhauser M., Kroh A., Mandic O., Piller W. E., GÖHlich U., Reuter M. \& Berning B. 2007. - Biogeographic responses to geodynamics: A key study all around the Oligo-Miocene Tethyan Seaway. Zoologischer Anzeiger 246: 241-256. https:// doi.org/10.1016/j.jcz.2007.05.001

Harzhauser M., Mandic O. \& Zuschin M. 2003. — Changes in Paratethyan marine molluscs at the Early/Midlle Miocene transition: diversity, palaeogeography and palaeoclimate. Acta Geologica Polonica 53 (4): 323-339.

Harzhauser M. \& Piller W. E. 2007. — Benchmark data of a changing sea-palaeogeography, palaeobiogeography and events in the Central Paratethys during the Miocene. Palaeogeography, Palaeoclimatology, Palaeoecology 253: 8-31. https://doi.org/10.1016/j. palaeo.2007.03.031

Hauer F. \& Stache G. 1863. - Geologie Siebenburgens. Braumuller, Wien, $636 \mathrm{p}$.

Hegedüs G. 1970. - Tortonai korallok Herendrol. Földtani Közlöny 100: 185-191.

Hegedüs G. \& JANKOVICH I. 1970. — Badenien korallzátony márkházaról. M. A. Földtani Intézet Évi Jelentéze 1970-röl: 39-53.

Hoernes R. 1878. - Ueber das Vorkommen des Genus Conus in den marinen Neogen Ablagerungen der öster.-ungar. Monarchie. Verhandlungen der Kaiserlich-Koniglichen Geologischen Reichsanstalt 9: 191-196.

Hoernes R. \& Auinger M. 1879-1891. - Die Gasteropoden der Meeres Ablagerungen der ersten und zweiten Miocaenen Mediterran-Stufein der Österreichisch-Ungarischen Monarchie. Abhandlungen der Geologischen Bundesanstalt 12, 382 p.

Hohenegger J., Ćorić S. \& Wagreich M. 2014. - Timing of the Middle Miocene Badenian stage of the Central Paratethys. Geologica Carpathica 65 (1): 55-66. https://doi.org/10.2478/ geoca-2014-0004

Hopley D. 2011. - Encyclopedia of Modern Coral Reefs - Structure, Form and Process. Springer Science, Business Media, 1205 p.

HÖRNES M. 1854. - Ergebnisse der Reise nach Ungarn und Siebenbürgen im Sommer 1854. Sitzungen der Kaiserlich-Königlichen Geologischen Reichsanstalt 5: 886-887. 
HÖRNES M. 1856. — Die fossilen Mollusken des tertiär-beckens von Wien. Abhandlungen der Kaiserlich-Königlichen Geologischen Reichsanstalt 3: 1-736.

Huyghe D., Merle M., Lartaud F., Cheype E. \& Emmanuel L. 2012. - Middle Lutetian climate in the Paris Basin: implications for a marine hotspot of paleobiodiversity. Facies 58: 587-604. https://doi.org/10.1007/s10347-012-0307-3

Iliescu O., Hinculov A. \& Hinculov L. 1968. - Basinul Mehadia, Studiu geologic si paleontologic. Memorii Institutului Geologic 9: 1-198.

JOVANOVIĆ J. 2011. - Badenian fauna of corals in vicinity of Negotin (NE Serbia). The 4th International workshop on the Neogene from the Central and South-Eastern Europe (NCSEE), Abstracts: 20.

Karami M. P., Leeuw A. De, Krijgsman W., Meijer P. Th. \& WORTEL M. J. R. 2011. - The role of gateways in the evolution of temperature and salinity of semi-enclosed basins: An oceanic box model for the Miocene Mediterranean Sea and Paratethys. Global and Planetary Change 79: 73-88. https://doi.org/10.1016/j. gloplacha.2011.07.011

KIESSLING W., FlÜgEL E. \& GolonKA J. 1999. — Paleoreef Maps: evaluation of a comprehensive database on Phanerozoic reefs. AAPG Bulletin 83 (10): 1552-1587.

Косн A. 1898. - Neuere Beobachtungen und Aufsamlungen in Felso-Lapugy. Földtani Közlöny 28: 209-226.

KocH A. 1900. — Die Tertiärbildungen des Beckens der siebenbürgischen Landesteile. II. Neogen Abteilung. Mittheilungen aus dem Jahrbuch der königlich ungarischen geologischen Anstalt, 370 p.

Kojumdgieva E. \& STRACHIMIRov B. 1960. - Tortonien, in Les fossiles de Bulgarie, vol. 7. Sofia, Académie des Sciences de Bulgarie, $317 \mathrm{p}$.

Kolosváry G. 1961. — Adatok Erdély mezozóos és neozóos korallfaunanájának ismeretéthez. Földtani Intézet Évi Jelentéze 1961 (2): 227-258

KovÁCS Z. \& BALÁZS P. 2015. — Conidae (Neogastropoda) assemblage from the Middle Miocene of the Făget Basin (Romania) in the collection of the Hungarian Natural History Museum, Budapest. Fragmenta Palaeontologica Hungarica 32: 11-48. https:// doi.org/10.17111/FragmPalHung.2015.32.11

Kováč M., Andreyeva-Grigorovich A., Bajraktarević Z., Brzobohatý R., Filipescu S., Fodor L., Harzhauser M., Nagymarosy A., Oszczypko N., Pavelić D., RÖGl F., Saftić B., Sliva L. \& STUdencKa B. 2007. - Badenian evolution of the Central Paratethys Sea: paleogeography, climate and eustatic sea-level changes. Geologica Carpathica 58 (6): 579-606.

Kováč M., HudÁčKová N., Halásová E., Kováčova M., Holcová K., OszCZypKo-Clowes M., BÁldi K., Less G., Nagymarosy A., Ruman A., KluČiar T. \& Jamrich M. 2017. — The Central Paratethys palaeoceanography: a water circulation model based on microfossil proxies, climate, and changes of depositional environment. Acta Geologica Slovaca 9 (2): 75-114.

KuCERA M. 2009. - Determination of past sea temperatures, in Steele J. H., Turekian K. K. \& ThOrpe S. A. (eds), Encyclopedia of Ocean Sciences. Academic Press: 98-113.

KÜHN O. 1963. - Korallensteinkerne im österreichischen Miozän. Annalen des Naturhistorischen Museums Wien 66: 101-112.

KÜHN O. 1925. - Die Korallen des Miocäns von Eggenburg. Abhandlungen der Geologischen Bundensanstalt 22: 1-8.

LAMARCK J.-B. M. DE 1816. - Histoire naturelle des animaux sans vertèbres. Tome second. Verdière, Paris, 568 p. https://doi. org/10.5962/bhl.title.12712

Landau B., Harzhauser M. \& Beu A. G. 2009. - A Revision of the Tonnoidea (Caenogastropoda, Gastropoda) from the Miocene Paratethys and their palaeobiogeographic implications. Jahrbuch der Geologische Bundesanstalt 149 (1): 61-109.

lauriat-Rage A., Brébion P., Buge E., Chaix C., Chevalier M., Margerel J.-P. , Pajaud D., Pouit D., Roman J. \& Viaud J. M. 1989. - Le gisement redonien (Pliocène) de la Marnière
(La Limouzinière, Loire-Atlantique) - Biostratigraphie, paléobiologie, affinités paléobiogéographiques. Géologie de la France 1-2: 117-152.

Leprieur F., Descombes P., Gaboriau T., Cowman P. F., ParRavicini V., Kulbicki M., Melian C. J., de Santana C. N., Heine C., Mouillot D., Bellwood D. R., Pellissier L. C. 2016. - Plate tectonics drive tropical reef biodiversity dynamics. Nature Communications 7, 11461, 8 p. https://doi.org/10.1038/ ncomms 11461

LESSON R. P. 1831-1835. - Illustrations de zoologie ou recueil de figures d'animaux peintes d'après nature. Paris, Arthus-Bertrand, 60 pls. https://doi.org/10.5962/bhl.title.35614

LiNK H. F. 1807. - Beschreibung der Naturalien. Sammlungen Universität Rostok 3: 161-165.

Macovei G. 1909. - Basenul terțiar de la Bahna (Mehedinți). Anuarul Institului Geologic al României 3: 57-164.

MARINESCU F. 1972. - Néogène de la dépression de Transylvanie, du Banat et du Bassin dacique. Guide de l'excursion de la Ve Réunion du groupe de travail pour la Paratéthys. Institut géologique, Guide de l'excursion 9, $112 \mathrm{p}$.

MARINESCU F. \& POPESCU G. 1978. — Lévolution néogène des bassins intramontagneux du secteur occidental des Carpates méridionales. Revue roumaine de Géologie, Géophysique et Géographie, Géologie 22: 61-70.

MAYER K. 1864. - Die Tertiär-Fauna der Azoren und Madeiren. Selbstverlage des Autors, Zürich, 107 p.

MAYER-EYMAR K. 1883. - Die Versteinerungen der tertiären Schichten von der westlichen Insel im Birketel-Qurun See, in Zittel K. A. (ed.), Paleontographica, t. XXX, 1er volume, II, Pal. Teil.: 67-77. https://www.biodiversitylibrary.org/item/109782

MerLe D. 2008. - Le Lutétien: un exemple de point-chaud de la paléobiodiversité, in MERLE D. (coord.), Stratotype Lutétien. Muséum national d'Histoire naturelle, Paris; Biotope, Mèze; BRGM, Orléans: 174-181.

MeZnerics I. 1944. - Ditrupa-reste aus Ungarns. Annales historiconaturales Musei nationalis hungarici 37: 40-47.

MiCHELIN J. L. H. 1840-1847. - Iconographie zoophytologique. Description par localités et terrains des polypiers fossiles de France et pays environnants. P. Bertrand éditeur, Paris, 348 p. https:// doi.org/10.5962/bhl.title.11504

Michelin J. L. H. 1850. — Description d'une nouvelle espèce de caryophyllie. Revue et Magasin de Zoologie, 2e série, 2: 238-239. https://biodiversitylibrary.org/page/2339969

MichelotTI I. 1838. - Specimen zoophytologiae diluvianae. Aug. Taurinorum: Heredes Seb. Botta, Turin, $227+9$ p. https://doi. org/10.5962/bhl.title.11568

MienIS H. K. 1975. - A note on the Miocene Neritopsis from Lapugy, Romania. Soosiana 3: 35, 36.

Mihaljević M., Korpanty C., Renema W., Welsh K. \& PanDOLFI J. M. 2017. — Identifying patterns and drivers of coral diversity in the Central Indo-Pacific marine biodiversity hotspot. Paleobiology 43 (3): 343-364. https://doi.org/10.1017/ pab.2017.1

Milne-Edwards H. \& Haime J. 1848-1851. - Recherches sur la structure et la classification des Polypiers récents et fossiles. (vol. 16: 1851: Poritidae). Annales des Sciences naturelles, série 3, 16: 21-70

Milne-Edwards H. \& Haime J. 1851. - Monographie des polypiers fossiles des terrains paléozoïques. Archives du Muséum d'Histoire naturelle 5: 1-200 + 201-502.

Milne-EdWARDS H. \& HAIME J. 1857-1860. — Histoire naturelle des coralliaires ou polypes proprement dits. Librairie encyclopédique de Roret, Paris, t. 1 (1857: VIII + 1-95) ; 2 (1857: 1-268); 3 (1860: 1-219); atlas (1857: 31 pls). https://doi.org/10.5962/ bhl.title. 11574

MOISESCU G. 1955. — Date noi asupra faunei de molluște si brachiopode tortoniene de la Lăpugiu superior. Revista Universității C. I. Parhon şi a Politehnicii București 8: 255-286. 
MoisesCU G. \& POPESCU G. 1980. — Chattian-Badenian biochronology in Romania by means of molluscs. Anuarul Institutului de Geologie și Geofizica 56: 205-224.

MuCKENHUBER L. 1964. — Miozän-Korallen des Wiener Beckens. Sitzungsberichte der Akademie der Wissenschaften mathematischnaturwissenschaftliche Klasse 173: 301-308.

Neugeboren L. 1847. — Uber die Foraminiferen des Tegels von Felso-Lapugy. Berichte über die Mittheilungen von Freunden der Naturwissenschaften in Wien 2 (10): 163, 164.

NeUGEBOREN L. 1849-1850. - Foraminiferen von Felsö Lapugy unweit Dobra im Carlsburger District. Verhandlungen und Mittheilungen des Siebenbürg für Naturwissenschaften 2: 45-48. https:// biodiversitylibrary.org/page/11525282

NeUGebOREN L. 1851. - Foraminiferen von Ober Lapugy. Verhandlungen und Mittheilungen des Siebenbürg für Naturwissenschaften 2 118-123 (https://biodiversitylibrary.org/page/11525376), 124135 (https://biodiversitylibrary.org/page/11525382), 140-145 (https://biodiversitylibrary.org/page/11525601).

NEUGEBOREN L. 1852. — Foraminiferen von Ober-Lapugy beschriben und nach des Naturgezeichnet. Verhandlungen und Mittheilungen des Siebenbürg für Naturwissenschaften 3: 34-42 (https:// biodiversitylibrary.org/page/11525730), 50-59 (https://biodiversitylibrary.org/page/11525746).

Neugeboren L. 1856a. — Beitrage zur Kenntniss der Tertiär-Mollusken aus dem Tegelgebilde von Ober-Lapugy. Verhandlungen und Mittheilungen des Siebenbürg für Naturwissenschaften 7 (1): 107-116. https://biodiversitylibrary.org/page/11526791

Neugeboren L. 1856b. — Die Foraminiferen aus der Ordnung der Stichostegier von Ober-Lapugy in Siebenbürgen. Denkschriften Österreichische Akademie der Wissenschaften (Mathematisch-Naturwissenschaftliche Klasse) 12 (2): 65-108. https://biodiversitylibrary. org/page/33415737

NEUGEBOREN L. 1856c. — Über die Foraminiferen aus der Ordnung der Stichostegier von Ober-Lapugy. Sitzungsberichte der Akademie der Wissenschaften (Naturwissenschaftliche Klasse) 19: 333-335. https://biodiversitylibrary.org/page/36377968

NeugEBOREN L. 1860. - Berichtigungen zu den in den Jahrgängen 1, 2 und 3 der Verhandlungen und Mittheilungen über Foraminiferen von Ober-Lapugy erschienenen Aufsätze. Verhandlungen und Mittheilungen des Siebenbürg für Naturwissenschaften 11 (4): 55-57. https://biodiversitylibrary.org/page/11528647

Neugeboren L. 1869. - Neue miocene Spiroloculinen aus dem Tegel von Ober-Lapugy. Verhandlungen und Mitteilungen des Siebenbürgischen Vereins für Naturwissenschaften zu Hermannstadt 20: 26-28. https://biodiversitylibrary.org/page/33600436

Neugeboren L. 1876. - Systematisches Verzeichniss der in den Miocän-Schichten bei Ober-Lapugy in Siebenbürgen vorkommenden fossilen Korallen. Verhandlungen und Mittheilungen des Siebenbürg für Naturwissenschaften 27: 41-51. https://biodiversitylibrary.org/page/34052286

Neugeboren L. 1878. - SystematischesVerzeichniss der in dem Tegelgebilde von Ober Lapugy vorkommenden Conchiferen. Verhandlungen und Mittheilungen des Siebenbürg für Naturwissenschaften 29: 109-136. https://biodiversitylibrary.org/page/34056202

Nicorici E. \& SAGATOVICI A. 1973. - Studiul faunei Badenian superioare de la Minişul de Sus (Bazinul Zarand). Anuarul Institutul Geologic 40: 112-194

NițUlESCU O. 1930. - Contribuții la studiul geologic al regiuni Lăpugiu-de-sus (Hunedoara). Revista Muzeului de GeologieMineralogie 4 (1): 27-43.

OOSTERBAAN A. F. F. 1990. - Notes on a collection of Badenian (Middle Miocene) corals from Hungary in the National Muzeum of Natural History at Leiden (The Nederlandens). Contributions to Tertiary and Quaternary Geology 27 (1): 3-15.

OrBIGNY A. C. V. D. D' 1852. - Prodrome de paléontologie stratigraphique universelle des animaux mollusques et rayonnés, faisant suite au cours élémentaire de paléontologie et de géologie stratigraphiques. Vol. 3. Masson, Paris, 190 p. https://www.biodiversitylibrary.org/item/98989
Ortmann A. 1890. — Die Morphologie des Skeletts der Steinkorallen in Beziehung zur Kolonienbildung. Zeitschrift fürWissenshatliche Zoologie 50: 278-316. https://biodiversitylibrary.org/ page/42561641

OSASCO E. 1897. - Di alcuni corallari miocenici del Piemonte. Atti della Accademia delle scienze di Torino 32: 640-652. https:// biodiversitylibrary.org/page/12212169

PACAUd J.-M. 2003. - First fossil records of the Recent Ovulid genus Pseudocypraea Schilder, 1927 (Mollusca, Gastropoda) with description of a new species. Geodiversitas 25 (3): 451-462.

PALLAS P. S. 1766. - Elenchus zoophytorum sistens generum adumbrationes generaliores et specierum cognitarum succintas descriptiones, cum selectis auctorum synonymis. Hagae conitum $n^{\circ} 176 \mathrm{~b}$, Francfort-sur le-Main, 451 p. https://doi.org/10.5962/bhl.title.6595

PĂUCA M. 1936. - Le bassin néogène de Beiuş. Annuaire de l'Institut géologique de Roumanie 17: 133-223.

PERRIN C. \& BoselLINI F. 2012. - Paleobiogeography of scleractinian reef corals: Changing patterns during the Oligocene-Miocene climatic transition in the Mediterranean. Earth-Science Reviews 111: 1-24. https://doi.org/10.1016/j.earscirev.2011.12.007

PERRIN C. 2002. - Tertiary: The emergence of modern reef ecosystems, in Kiessling W., Flügel E. \& Golonka J. (eds), Phanerozoic Reef Patterns. SEPM, Special Publications 72: 587-621. https://doi.org/10.2110/pec.02.72.0587

Perrin C. \& Kiessling W. 2010. — Latitudinal trends in OligoMiocene reef patterns and their relationship to climate, in MUTTI M., Piller W. E. \& Betzler C. (eds), Carbonate systems during the Oligocene-Miocene climatic transition. IAS Special Publication 42: 17-34

Petrescu I., Mészaros N., Chira C. \& Filipescu S. 1990. Lower Badenian paleoclimate at Lăpugiu de Sus (Hunedoara County), on account of paleontological investigation. Studia Universitatis Babes-Bolyai, Geologia 35 (2): 13-22.

Piller W. \& KleEmann K. 1991. - Middle Miocene reefs and related facies in Eastern Austria. I. Vienna basin. VIth International Symposium on Fossil Cnidaria including Archaeocyatha and Porifera, Münster 1991, Excursion B4 Guidebook: 2-28.

Piller W. M., Harzhauser M. \& Mandic O. 2007. - Miocene Central Paratethys stratigraphy - Current status and future directions. Stratigraphy 4: 151-168.

PISERA A. 1996. - Miocene reefs of the Paratethys: a review. SEPM, Society for Sedimentary Geology, Concepts in Sedimentology and Paleontology 5: 97-104. https://doi.org/10.2110/csp.96.01.0097

PoP E. I. 1960. — Étude géologique du bassin de Mehadia. Anuarul Comitetului Geologic 29-30: 119-141.

Popa M. V., Duma A., SĂPlĂCAN A. 2014. — Badenian gastropods from the collections of the Mures County Museum. Analele Stiintifice ale Universitații "Alexandru Ioan Cuza" din Iaşi Seria Geologie 60 (2): 5-30.

PopesCU G. \& CRiHAn I. M. 2005. — Middle Miocene Foraminifera from Romania: order Buliminida, Part 1. Acta Palaeontologica Romaniae 5: 379-396.

POURTALÈs L. F. DE 1871. — Deep-sea corals. Illustrated Catatalogue of the Musem of Comparative Zoölogy, at Harvard College 4: 1-93. https://biodiversitylibrary.org/page/4200163

RANGHEARD Y. 1969. - Étude géologique des îles d'Ibiza et de Formentera (Baléares). Thèse de Sciences, Université de Besançon, France, $478 \mathrm{p}$.

RENEMA W. 2011. - Biodiversity hotspots were cold during the Eocene. Berichte der Geologischen Bundesanstalt 85: 136.

Reuss A. E. VON 1847. — Die fossilen Polyparien des wiener Tertiärbeckens. Ein monographischer Versuch, in HAIDINGER W. (ed.) Naturwissenschaften Abhandlungen 2: 1-109.

REUSS A. E. VON 1854. - Beiträge zur geognostichen Kenntniss Mährens. Jahrbuch der Kaiserlich Königlichen Geologischen Reichsanstalt 4: 659-765. https://biodiversitylibrary.org/page/48762599

REuss A. E. VON 1860. — Die marinen Tertiärschichten Böhmens und ihre Versteinerungen. Sitzungsberichte der Kaiserlichen Aka- 
demie der Wissenschaften Wien 39: 207-285. https://biodiversitylibrary.org/page/34807863

REUSS A. E. vON 1864. - Zur Fauna des deutschen Oberoligocäns. Sitzungsberichte der Kaiserlichen Akademie der Wissenschaften Wien 50: 614-687. https://biodiversitylibrary.org/page/6445706

Reuss A. E. vON 1867. — Die fossile Fauna der Steinsalzablagerung von Wieliczka in Galizien. Sitzungsberichte der Kaiserlichen Akademie der Wissenschaften Wien 55: 17-182. https://biodiversitylibrary.org/page/39323016

Reuss A. E. VON 1869. - Die fossilen Anthozoen und Bryozoen der Schichtengruppe von Crosara. Denkschriften der Kaiserlichen Akademie der Wissenschaften Wien (Mathematisch-Naturwissenschaftliche Klasse) 29: 215-298. https://biodiversitylibrary. org/page/28668262

REUSS A. E. VON 1871. — Die Fossilen Korallen des Österreichisch-Ungarischen Miocäns. Denkschriften der Kaiserlichen Akademie der Wissenschaften Wien (Mathematisch-Naturwissenschaftliche Klasse) 31: 197-270. https://biodiversitylibrary. org/page/27923011

Reuter M. \& Piller W. E. 2011. — Volcaniclastic events in coral reef and seagrass environments: evidence for disturbance and recovery (Middle Miocene, Styrian Basin, Austria). Coral Reefs 30: 889-899. https://doi.org/10.1007/s00338-011-0798-3

Reuter M. \& Piller W. E. 2014. - Neogene of the Styrian Basin. Berichte des Institutes für Erdwissenschaften Karl-FranzensUniversität Graz 20 (2): 22-43.

Reuter M., Piller W. E. , Erhart C. 2012. — A Middle Miocene carbonate platform under silici-volcaniclastic sedimentation stress (Leitha Limestone, Styrian Basin, Austria). Depositional environments, sedimentary evolution and palaeoecology. Palaeogeography, Palaeoclimatology, Palaeoecology 350-352: 198-211. https://doi.org/10.1016/j.palaeo.2012.06.032

Riegl B. \& Piller W. E. 2000a. - Reefs and coral carpets in the Miocene Paratethys (Badenian, Leitha Limestone, Austria), in Moosa M. K., Soemodihardjo S., Soegiarto A., Romimohtarto K., Nontji A., Soekarno \& Suharsono (eds), Proceedings of the $9^{\text {th }}$ International Coral Reef Symposium (Bali, Indonesia) 1: 211-216.

Riegl B. \& Piller W. E. 2000b. - Biostromal coral facies-a Miocene example from the Leitha Limestone (Austria) and its actualistic interpretation. Palaios 15: 399-413. https://doi. org/10.1669/0883-1351(2000)015<0399:BCFAME>2.0.CO;2

RÖGL F. 1998. - Palaeogeographic considerations for Mediterranean and Paratethys seaways (Oligocene to Miocene). Annalen des Naturhistorischen Museums in Wien 99A: 279-310.

RÖGL F. 1999. - Mediterranean and Paratethys. Facts and hypotheses of an Oligocene to Miocene paleogeography (short overview). Geologica Carpathica 50 (4): 339-349.

Rögl F., Ćorić S., Harzhauser M., Jimenez-Moreno G., Kroh A., Schultz O., Wessely G. \& Zorn I. 2008. - The Middle Miocene Badenian stratotype at Baden-Sooss (Lower Austria). Geologica Carpathica 59 (5): 367-374.

Rögl F., Ćorić S., Hohenegger J., Pervesler P., Roetzel R., Scholger R., Spezzaferri S. \& Stingl K. 2007. Cyclostratigraphy and transgressions at the Early/Middle Miocene Karpatian/Badenian) boundary in the Austrian Neogene Basins (Central Paratethys). Scripta Facultatis Scientarium Naturalium Universitatis Masarykianae Brunensis, Geology 36: 7-13.

RonieWICZ E. \& STOlarski J. 1991. - Miocene Scleractinia from the Holy Cross Mountains, Poland; Part 2 - Archaeocoeniina, Astraeina, and Fungiina. Acta Geologica Polonica 41 (1-2): 69-83.

RoszKowska M. 1932. - Korale mioceńskie polski. Annales Societatis Geologorum Poloniae 8 (1): 97-171.

Rus M. \& Popa M. V. 2008. - Taxonomic notes on the Badenian Corals from Lăpugiu de Sus (Făget Basin, Romania). Acta Palaeontologica Romaniae 6: 325-337.
SAINT MARTIN J.-P. 1990. — Les formations récifales coralliennes du Miocène supérieur d'Algérie et du Maroc. Muséum national d'Histoire naturelle, Paris, 351 p. (Mémoires du Muséum national d'Histoire naturelle, sér. C - Sciences de la Terre (1950-1992); 56).

SAINT MARTIN J.-P. 1996. - Messinian coral reefs of Western Oranie (Algeria). SEPM, Concepts in Sedimentology and Paleontology series 5: 239-246. https://doi.org/10.2110/csp.96.01.0239

Saint Martin J.-P. \& Cornée J. J. 1996. — The Messinian Reef Complex of Melilla, Northeastern Rif, Morocco. SEPM, Concepts in Sedimentology and Paleontology Series 5: 227-237. https://doi. org/10.2110/csp.96.01.0227

Saint Martin J.-P., Müller P., Moissette P. \& Dulai A. 2000. Coral microbialite environment in a Middle Miocene reef of Hungary. Palaeogeography, Palaeoclimatology, Palaeoecology 160: 179-191. https://doi.org/10.1016/S0031-0182(00)00065-1

Saint Martin J.-P., Merle D., Cornée J. J. , Filipescu S., Saint MARTIN S. \& BUCUR I. 2007. - Les constructions coralliennes du Badénien (Miocène moyen) de la bordure occidentale de la dépression de Transylvanie (Roumanie). Comptes Rendus Palevol 6: 37-45. https://doi.org/10.1016/j.crpv.2006.07.006

Sanders M. T., Merle D. \& Villier L. 2015. — The molluscs of the "Falunière" of Grignon (Middle Lutetian, Yvelines, France): quantification of lithification bias and its impact on the biodiversity assessment of the Middle Eocene of Western Europe. Geodiversitas 37 (3): 345-365. https://doi.org/10.5252/g2015n3a4

SCHOLZ G. 1970. — A Visegradi Fekete-hegy Tortonai korallok faunaja. Földtani Közlöny 100: 192-206.

SCHouppé A. 1949. - Die Fauna des Steinbruches von Retznei bei Ehrenhausen. Mitteilungen des naturwissenschaftlichen Vereins für Steiermark 77-78: 142-144.

Schmid H. P., Harzhauser M. \& KrOH A. 2001. - Hypoxic events on a Middle Miocene carbonate platform of the Central Paratethys (Austria, Badenian, $14 \mathrm{Ma}$ ). Annalen des Naturhistorischen Museums in Wien 102A: 1-50.

SCHUHMACHER H. \& Zibrowius H. 1985. - What is hermatypic? a redefinition of ecological groups in corals and other organisms. Coral Reefs 4: 1-9. https://doi.org/10.1007/BF00302198

SCHWEIGGER A. F. 1819. - Beobachtungen auf naturhistorischen Reisen: Anatomisch-physiologische Untersuchung über Corallen; nebst ein Anhange, Bemerkungen über die Bernstein enthaltend. Reimer, Berlin, 127 p. https://doi.org/10.5962/bhl.title.14416 SISMONDA E. 1871. - Matériaux pour servir à la paléontologie du terrain tertiaire du Piémont. Memorie della Reale Accademia della Scienze di Torino, série 2, 25: 257-361. https://biodiversitylibrary. org/page/36389689

Sremac J., Bošnjak Makovec M., Vrsaljko D., Karaica B., Tripalo K.; Fio Firi K., MajSTORović Bušić A. \& MarjanaC T. 2015. - Reefs and bioaccumulations in the Miocene deposits of the North Croatian Basin - Amazing diversity yet to be described. The Mining-Geology-Petroleum Engineering Bulletin 2015: 19-29. https://doi.org/10.17794/rgn.2016.1.2

Stanley G. D. JR \& Cairns S. D. 1988. - Constructional Azooxanthellate Coral Communities: An Overview with Implications for the Fossil Record. Palaios 3: 233-242. https://doi. org/10.2307/3514533

STOLARSKI J. 1991. - Miocene Scleractinia from the Holy Cross Mountains, Poland; Part 1 - Caryophylliidae, Flabellidae, Dendrophylliidae, and Micrabaciidae. Acta Geologica Polonica 41 (1-2): 37-67.

STUR D. 1863. - Bericht über die geologische Übersichtaufnahme des südwestlichen Siebenbürgenim Sommer 1860. Jahrbuch der kaiserlich-königlichen Geologischen Reichsanstalt 13 (1): 33-120. https://biodiversitylibrary.org/page/35580730

ŞuRARU N. \& PAPp C. 1993. - Einige Angabenüber den Mikrofossilen inhalt der Ablagerungen des Badeniens von Lăpugiu de Sus (Kreis Hunedoara). Studia Universitatis Babeș-Bolyai, Geologia 38 (2): 27-36. 
ȚABĂRĂ D. \& CHIRILĂ G. 2012. — Palaeoclimatic estimation from Miocene of Romania, based on palynological data. Carpathian Journal of Earth and Environmental Sciences 7 (2): 195-208.

TĂMAS A., TÃMAS T. M. \& POPA M. V. 2013. - Badenian small gastropods from Lăpugiu de Sus (Făget Basin, Romania). Rissoidae Family. Acta Palaeontologica Romaniae 9: 57-66.

TiȚA R. 1999. - Biostratigraphical study of the Badenian deposits from Delinești (România). Travaux du Muséum national d'Histoire naturelle Grigore Antipa 41: 473-486.

TiȚA R. 2000. - Study on Miocene corals (Scleractinian) from Delinești (România). Travaux du Muséum national d'Histoire naturelle Grigore Antipa 42: 373-382.

TiȚA R. 2007. - Comments on the Badenian fauna (Middle Miocene) from Bahna (Southern Carpathians, Romania). Travaux du Muséum national d'Histoire naturelle Grigore Antipa 50: 543-554.

VAughan T. W. 1919. - Fossils corals from Central America, Cuba and Porto-Rico, with an account of the American Tertiary, Pleistocene and Recent Coral Reefs. United States National Museum Bulletin 103: 189-524. https://doi.org/10.5962/bhl.title.46527

VAUGHAN T. W. \& WeLLS J. W. 1943. — Revision of the suborders, families and genera of the Scleractinia. Geological Society of America, Special Papers 44: XV + 363 p. https://doi.org/10.1130/SPE44

VÁVRA N. 1977. - Bryozoa tertiaria, in ZAPFE H. (ed.), Catalogus Fossilium Austriae. Vb/3: 1-189.

Veron J. E. N. 2000a. - Corals of the World. Volume 1. Australian Institute of Marine Science, $463 \mathrm{p}$.

Veron J. E. N. 2000b. - Corals of the World. Volume 2. Australian Institute of Marine Science, $429 \mathrm{p}$.

VerON J. E. N. 2000c. - Corals of the World. Volume 3. Australian Institute of Marine Science, $490 \mathrm{p}$.

VerRILl A. E. 1870. - Review of the corals and polyps of the west coast of America. Transactions of the Connecticut Academy of Arts and Sciences 1, 2: 377-558. https://biodiversitylibrary. org/page/27729638

Vertino A., Stolarski J., Bosellini F. \& TaViani M. 2014. Mediterranean corals through time: from Miocene to Present, in Goffredo S. \& DubinSKY Z. (eds), The Mediterranean Sea: Its History and Present Challenges. Springer Science+Business Media Dordrecht: 257-272. https://doi.org/10.1007/978-94007-6704-1_14

Weisbord N. E. 1968. - Some late Cenozoic stony corals from northern Venezuela. Bulletins of American Paleontology 55: 6-267. https://biodiversitylibrary.org/page/10653928
WELLS J. W. 1933. - Corals of the Cretaceous of the Atlantic and Gulf coastal plains and western interior of the United States. Bulletins of American Paleontology 18 (67): 85-288. https://biodiversitylibrary.org/page/30409254

Wells J. W. 1937. - New genera of Mesozoic and Cenozoic corals. Journal of Paleontology 11: 73-77. https://www.jstor.org/ stable/1298314

Wells J. W. 1956. - Scleractinia, in Moore R. C. (ed.), Treatise on Invertebrate Paleontology. Geological Society of America, part F: 328-443.

Wiedl T., Harzhauser M. \& Piller W. E. 2012. - Facies and synsedimentary tectonics on a Badenian carbonate platform in the southern Vienna Basin (Austria, Central Paratethys). Facies 58: 523-548. https://doi.org/10.1007/s10347-011-0290-0

Wiedl T., Harzhauser M., Kroh A., Ćorić S. \& Piller W. E. 2013. - Ecospace variability along a carbonate platform at the northern boundary of the Miocene reef belt (Upper Langhian, Austria). Palaeogeography, Palaeoclimatology, Palaeoecology 370: 232-246. https://doi.org/10.1016/j.palaeo.2012.12.015

Willis K., Gillson K. \& KNAPP S. 2006. — Biodiversity hotspots through time: an introduction. Philophical Transactions of the Royal Society B 362: 169-174. https://doi.org/10.1098/rstb.2006.1976

Wood S. 1844. - Descriptive catalogue of the zoophytes from the Crag - Annals and Magazine of Natural History 13: 10-21. https://doi.org/10.1080/03745484409442561

Zachos J., Pagani M., Sloan L., Thomas E. \& Billups K. 2001. Trends, rythms and aberrations in the global climate $65 \mathrm{Ma}$ to present Science 292: 686-693. https://doi.org/10.1126/science.1059412

ZeIgler 2014. - Competition, in ZeIGLer D. (ed.), Evolution Components and Mechanisms. Academic Press: 31-38.

Zibrowius H. 1988. - Mise au point sur les sléractiniaires comme indicateurs de profondeur (Cnidaria: Anthozoa). Géologie méditerranéenne 15 (1): 27-47.

Zlatarski V. N. \& Martinez Estalella N. 1982. — Les Scléractiniaires de Cuba avec des données sur les organismes associés. Éditions de l'Académie Bulgare des Sciences, $471 \mathrm{p}$.

Zuschin M., Harzhauser M. \& Mandic O. 2011. - Disentangling palaeodiversity signals from a biased sedimentary record: an example from the Early to Middle Miocene of Central Paratethys Sea, in MCGowan A. J. \& SMITH A. B. (eds) Comparing the Geological and Fossil Records: Implications for Biodiversity Studies. Geological Society, London, Special Publications 358: 123 139. https://doi.org/10.1144/SP358.9

Soumis le 9 janvier 2018 accepté le 27 mars 2018 publié le 19 juillet 2018. 